\title{
From Pseudorandom Walk to Pseudo-Brownian Motion: First Exit Time from a One-Sided or a Two-Sided Interval
}

\author{
Aimé Lachal ${ }^{1,2}$ \\ ${ }^{1}$ Université de Lyon, Institut Camille Jordan, CNRS UMR5208, France \\ ${ }^{2}$ Institut National des Sciences Appliquées de Lyon Pôle de Mathématiques, Bâtiment Léonard de Vinci, \\ 20 Avenue Albert Einstein, 69621 Villeurbanne Cedex, France
}

Correspondence should be addressed to Aimé Lachal; aime.lachal@insa-lyon.fr

Received 20 May 2013; Accepted 14 August 2013; Published 26 March 2014

Academic Editor: M. Lopez-Herrero

Copyright (C) 2014 Aimé Lachal. This is an open access article distributed under the Creative Commons Attribution License, which permits unrestricted use, distribution, and reproduction in any medium, provided the original work is properly cited.

\begin{abstract}
Let $N$ be a positive integer, $c$ a positive constant and $\left(\xi_{n}\right)_{n>1}$ be a sequence of independent identically distributed pseudorandom variables. We assume that the $\xi_{n}$ 's take their values in the discrete set $\{-N,-N+1, \ldots, N-1, N\}$ and that their common pseudodistribution is characterized by the (positive or negative) real numbers $\mathbb{P}\left\{\xi_{n}=k\right\}=\delta_{k 0}+(-1)^{k-1} c\left(\begin{array}{c}2 N \\ k+N\end{array}\right)$ for any $k \in\{-N,-N+1, \ldots, N-1, N\}$. Let us finally introduce $\left(S_{n}\right)_{n \geq 0}$ the associated pseudorandom walk defined on $\mathbb{Z}$ by $S_{0}=0$ and $S_{n}=\sum_{j=1}^{n} \xi_{j}$ for $n \geq 1$. In this paper, we exhibit some properties of $\left(S_{n}\right)_{n \geq 0}$. In particular, we explicitly determine the pseudodistribution of the first overshooting time of a given threshold for $\left(S_{n}\right)_{n \geq 0}$ as well as that of the first exit time from a bounded interval. Next, with an appropriate normalization, we pass from the pseudorandom walk to the pseudo-Brownian motion driven by the high-order heat-type equation $\partial / \partial t=(-1)^{N-1} c \partial^{2 N} / \partial x^{2 N}$. We retrieve the corresponding pseudodistribution of the first overshooting time of a threshold for the pseudo-Brownian motion (Lachal, 2007). In the same way, we get the pseudodistribution of the first exit time from a bounded interval for the pseudo-Brownian motion which is a new result for this pseudoprocess.
\end{abstract}

\section{Introduction}

Throughout the paper, we denote by $\mathbb{Z}$ the set of integers, by $\mathbb{N}$ that of nonnegative integers, and by $\mathbb{N}^{*}$ that of positive integers: $\mathbb{Z}=\{\ldots,-1,0,1, \ldots\}, \mathbb{N}=\{0,1,2, \ldots\}, \mathbb{N}^{*}=\{1$, $2, \ldots\}$. More generally, for any set of numbers $E$, we set $E^{*}=$ $E \backslash\{0\}$.

Let $N$ be a positive integer, $c$ a positive constant, and set $\kappa_{N}=(-1)^{N-1}$. Let $\left(\xi_{n}\right)_{n \in \mathbb{N}^{*}}$ be a sequence of independent identically distributed pseudorandom variables taking their values in the set of integers $\{-N,-N+1, \ldots,-1,0,1, \ldots, N-$ $1, N\}$. By pseudorandom variable, we mean a measurable function defined on a space endowed with a signed measure with a total mass equaling the unity. We assume that the common pseudodistribution of the $\xi_{n}$ 's is characterized by the (positive or negative) real pseudo-probabilities $p_{k}=\mathbb{P}\left\{\xi_{n}=k\right\}$ for any $k \in\{-N,-N+1, \ldots, N-1, N\}$. The parameters $p_{k}$ sum to the unity: $\sum_{k=-N}^{N} p_{k}=1$.

Now, let us introduce $\left(S_{n}\right)_{n \in \mathbb{N}}$ the associated pseudorandom walk defined on $\mathbb{Z}$ by $S_{0}=0$ and $S_{n}=\sum_{j=1}^{n} \xi_{j}$ for $n \in \mathbb{N}^{*}$.
The infinitesimal generator associated with $\left(S_{n}\right)_{n \in \mathbb{N}}$ is defined, for any function $f$ defined on $\mathbb{Z}$, as

$$
\begin{aligned}
\mathscr{G}_{S} f(j) & =\mathbb{E}\left[f\left(\xi_{1}+j\right)\right]-f(j) \\
& =\sum_{k=-N}^{N} p_{k} f(j+k)-f(j), \quad j \in \mathbb{Z} .
\end{aligned}
$$

Here we consider the pseudorandom walk which admits the discrete $\mathrm{N}$-iterated Laplacian as a generator infinitesimal. More precisely, by introducing the so-called discrete Laplacian $\Delta$ defined, for any function $f$ defined on $\mathbb{Z}$, by

$$
\Delta f(j)=f(j+1)-2 f(j)+f(j-1), \quad j \in \mathbb{Z},
$$

the discrete $N$-iterated Laplacian is the operator $\Delta^{N}=$ $\underline{\Delta \circ \cdots \circ \Delta}$ given by

$$
\Delta^{N} f(j)=\sum_{k=-N}^{N}(-1)^{k+N}\left(\begin{array}{c}
2 N \\
k+N
\end{array}\right) f(j+k), \quad j \in \mathbb{Z} .
$$


We then choose the $p_{k}$ 's such that $\mathscr{G}_{S}=\kappa_{N} c \Delta^{N}$ which yields, by identification, for any $k \in\{-N,-N+1, \ldots,-1,1, \ldots, N-$ $1, N\}$,

$$
p_{k}=p_{-k}=(-1)^{k-1} c\left(\begin{array}{c}
2 N \\
k+N
\end{array}\right), \quad p_{0}=1-c\left(\begin{array}{c}
2 N \\
N
\end{array}\right) \text {. }
$$

When $N=1,\left(S_{n}\right)_{n \in \mathbb{N}}$ is the nearest neighbours pseudorandom walk with a possible stay at its current location; it is characterized by the numbers $p_{1}=p_{-1}=c$ and $p_{0}=$ $1-2 c$. Moreover, if $0<c<1 / 2$, then $p_{0}>0$; in this case, we are dealing with an ordinary symmetric random walk (with positive probabilities). If $c=1 / 2$, this is the classical symmetric random walk: $p_{1}=p_{-1}=1 / 2$ and $p_{0}=0$.

Actually, with the additional assumption that $p_{-k}=p_{k}$ for any $k \in\{-N,-N+1, \ldots, N-1, N\}$ (i.e., the $\xi_{n}$ 's are symmetric, or the pseudorandom walk has no drift), the $p_{k}$ 's are the unique numbers such that

$$
\begin{aligned}
\mathscr{G}_{S} f(j)= & \kappa_{N} c \widetilde{f}^{(2 N)}(j) \\
& + \text { terms with higher order derivatives, }
\end{aligned}
$$

where $\tilde{f}$ is an analytical extension of $f$ and $\tilde{f}^{(2 N)}$ stands for the $(2 N)$ th derivative of $\tilde{f}: \tilde{f}^{(2 N)}(x)=\left(\mathrm{d}^{2 N} \widetilde{f} / \mathrm{d} x^{2 N}\right)(x)$.

Our motivation for studying the pseudorandom walk associated with the parameters defined by (4) is that it is the discrete counterpart of the pseudo-Brownian motion as the classical random walk is for Brownian motion. Let us recall that pseudo-Brownian motion is the pseudo-Markov process $\left(X_{t}\right)_{t \geq 0}$ with independent and stationary increments, associated with the signed heat-type kernel $p(t ; x)$ which is the elementary solution of the high-order heat-type equation $\partial / \partial t=\kappa_{N} c \partial^{2 N} / \partial x^{2 N}$. The kernel $p(t ; x)$ is characterized by its Fourier transform:

$$
\mathbb{E}\left(\mathrm{e}^{i u X_{t}}\right)=\int_{-\infty}^{+\infty} \mathrm{e}^{i u x} p(t ; x) \mathrm{d} x=\mathrm{e}^{-c t u^{2 N}} .
$$

The corresponding infinitesimal generator is given, for any $C^{2 N}$-function $f$, by

$$
\begin{aligned}
\mathscr{G}_{X} f(x) & =\lim _{h \rightarrow 0^{+}} \frac{1}{h}\left[\mathbb{E}\left[f\left(X_{h}+x\right)\right]-f(x)\right] \\
& =\kappa_{N} c f^{(2 N)}(x) .
\end{aligned}
$$

The reader can find extensive literature on pseudo-Brownian motion. For instance, let us quote the works of Beghin et al. [1-20] and the references therein.

We observe that (5) and (7) are closely related to the continuous $N$-iterated Laplacian $\mathrm{d}^{2 N} / \mathrm{d} x^{2 N}$. For $N=2$, the operator $\Delta^{2}$ is the two-Laplacian related to the famous biharmonic functions: in the discrete case,

$$
\begin{aligned}
\Delta^{2} f(j)= & f(j+2)-4 f(j+1)+6 f(j) \\
& -4 f(j-1)+f(j-2), \quad j \in \mathbb{Z},
\end{aligned}
$$

and in the continuous case,

$$
\Delta^{2} f(x)=\frac{\mathrm{d}^{4} f}{\mathrm{~d} x^{4}}(x), \quad x \in \mathbb{R} .
$$

In the discrete case, it has been considered by Sato [21] and Vanderbei [22].

The link between the pseudorandom walk and pseudoBrownian motion is the following one: when normalizing the pseudorandom walk $\left(S_{n}\right)_{n \in \mathbb{N}}$ on a grid with small spatial step $\varepsilon^{2 N}$ and temporal step $\varepsilon$ (i.e., we construct the pseudoprocess $\left(\varepsilon S_{\left\lfloor t / \varepsilon^{2 N}\right\rfloor}\right)_{t \geq 0}$ where $\lfloor\cdot\rfloor$ denotes the usual floor function), the limiting pseudoprocess as $\varepsilon \rightarrow 0^{+}$is exactly the pseudoBrownian motion.

Now, we consider the first overshooting time of a fixed single threshold $a<0$ or $b>0$ ( $a, b$ being integers) for $\left(S_{n}\right)_{n \in \mathbb{N}}$ :

$$
\begin{aligned}
& \sigma_{a}^{-}=\min \left\{n \in \mathbb{N}^{*}: S_{n} \leq a\right\} \\
& \sigma_{b}^{+}=\min \left\{n \in \mathbb{N}^{*}: S_{n} \geq b\right\}
\end{aligned}
$$

as well as the first exit time from a bounded interval $(a, b)$ :

$$
\begin{aligned}
\sigma_{a b} & =\min \left\{n \in \mathbb{N}^{*}: S_{n} \leq a \text { or } S_{n} \geq b\right\} \\
& =\min \left\{n \in \mathbb{N}^{*}: S_{n} \notin(a, b)\right\}
\end{aligned}
$$

with the usual convention that $\min \emptyset=+\infty$. Hence, when $\sigma_{b}^{+}<+\infty, S_{\sigma_{b}^{+}-1} \leq b-1$ and $S_{\sigma_{b}^{+}} \geq b$, the overshoot at time $\sigma_{b}^{+}$ which is $S_{\sigma_{b}^{+}}-b$ can take the values $0,1,2, \ldots, N-1$, that is, $S_{\sigma_{b}^{+}} \in\{b, b+1, b+2, \ldots, b+N-1\}$. Similarly, when $\sigma_{a}^{-}<+\infty$, $S_{\sigma_{a}^{-}} \in\{a-N+1, a-N+2, \ldots, a\}$, and when $\sigma_{a b}<+\infty$, $S_{\sigma_{a b}} \in\{a, a-1, \ldots, a-N+1\} \cup\{b, b+1, \ldots, b+N-1\}$. We put $S_{b}^{+}=S_{\sigma_{b}^{+}}, S_{a}^{-}=S_{\sigma_{a}^{-}}$and $S_{a b}=S_{\sigma_{a b}}$.

In the same way, we introduce the first overshooting times of the thresholds $a<0$ and $b>0$ ( $a, b$ being now real numbers) for $\left(X_{t}\right)_{t \geq 0}$ :

$$
\tau_{a}^{-}=\inf \left\{t \geq 0: X_{t} \leq a\right\}, \quad \tau_{b}^{+}=\inf \left\{t \geq 0: X_{t} \geq b\right\}
$$

as well as the first exit time from a bounded interval $(a, b)$ :

$$
\begin{aligned}
\tau_{a b} & =\inf \left\{t \geq 0: X_{t} \leq a \text { or } X_{t} \geq b\right\} \\
& =\inf \left\{t \geq 0: X_{t} \notin(a, b)\right\}
\end{aligned}
$$

with the similar convention that $\inf \emptyset=+\infty$, and we set, when the corresponding time is finite,

$$
X_{a}^{-}=X_{\tau_{a}^{-}}, \quad X_{b}^{+}=X_{\tau_{b}^{+}}, \quad X_{a b}=X_{\tau_{a b}} .
$$

In this paper we provide a representation for the generating function of the joint distributions of the couples $\left(\sigma_{a}^{-}, S_{a}^{-}\right),\left(\sigma_{b}^{+}, S_{b}^{+}\right)$, and $\left(\sigma_{a b}, S_{a b}\right)$. In particular, we derive simple expressions for the marginal distributions of $S_{a}^{-}, S_{b}^{+}$, and $S_{a b}$. We also obtain explicit expressions for the famous "ruin pseudoprobabilities" $\mathbb{P}\left\{\sigma_{a}^{-}<\sigma_{b}^{+}\right\}$and $\mathbb{P}\left\{\sigma_{b}^{+}<\sigma_{a}^{-}\right\}$. The main tool employed in this paper is the use of generating functions.

Taking that the limit as $\varepsilon$ goes to zero, we retrieve the joint distributions of the couples $\left(\tau_{a}^{-}, X_{a}^{-}\right)$and $\left(\tau_{b}^{+}, X_{b}^{+}\right)$obtained in $[10,11]$. Therein, we used Spitzer's identity for deriving these distributions. Moreover, we obtain the joint distribution of 
the couple $\left(\tau_{a b}, X_{a b}\right)$ which is a new and an important result for the study of pseudo-Brownian motion. In particular, we deduce the "ruin pseudo-probabilities" $\mathbb{P}\left\{\tau_{a}^{-}<\tau_{b}^{+}\right\}$and $\mathbb{P}\left\{\tau_{b}^{+}<\tau_{a}^{-}\right\}$; the results have been announced without any proof in a survey on pseudo-Brownian motion [13], after a conference held in Madrid (IWAP 2010).

In $[11,17,18]$, the authors observed a curious fact concerning the pseudodistributions of $X_{a}^{-}$and $X_{b}^{+}$: they are linear combinations of the Dirac distribution and its successive derivatives (in the sense of Schwarz distributions). For instance,

$$
\frac{\mathbb{P}\left\{X_{b}^{+} \in \mathrm{d} z\right\}}{\mathrm{d} z}=\sum_{j=0}^{N-1} \frac{b^{j}}{j !} \delta_{b}^{(j)}(z) .
$$

The quantity $\delta_{b}^{(j)}$ is to be understood as the functional acting on test functions $\phi$ according to $\left\langle\delta_{b}^{(j)}, \phi\right\rangle=(-1)^{j} \phi^{(j)}(b)$. The appearance of the $\delta_{b}^{(j)}$, s in (15), which is quite surprising for probabilists, can be better understood thanks to the discrete approach. Indeed, the $\delta_{b}^{(j)}$,s come from the location at the overshooting time of $b$ for the normalized pseudorandom walk: the location takes place in the "cluster" of points $b, b+$ $\varepsilon, b+2 \varepsilon, \ldots, b+(N-1) \varepsilon$.

In order to facilitate the reading of the paper, we have divided it into three parts:

Part I-some properties of the pseudorandom walk

Part II-first overshooting time of a single threshold

Part III-first exit time from a bounded interval.

The reader will find a list of notations in Table 2 which is postponed to the end of the paper.

\section{Part I-Some Properties of the Pseudorandom Walk}

2.1. Pseudodistribution of $\xi_{1}$ and $S_{n}$. We consider the pseudorandom walk $\left(S_{n}\right)_{n \in \mathbb{N}}$ related to a family of real parameters $\left\{p_{k}, k \in\{-N, \ldots, N\}\right\}$ satisfying $p_{k}=p_{-k}$ for any $k \in\{1, \ldots, N\}$ and $\sum_{k=-N}^{N} p_{k}=1$. Let us recall that the infinitesimal generator associated with $\left(S_{n}\right)_{k \geq 0}$ is defined by

$$
\begin{aligned}
\mathscr{G}_{S} f(j) & =\sum_{k=-N}^{N} p_{k} f(j+k)-f(j) \\
& =\left(p_{0}-1\right) f(j)+\sum_{k=1}^{N} p_{k}[f(j+k)+f(j-k)] .
\end{aligned}
$$

In this section, we look for the values of $p_{k}, k \in\{-N, \ldots, N\}$, for which the infinitesimal generator $\mathscr{G}$ is of the form (5). Next, we provide several properties for the corresponding pseudorandom walk.

Suppose that $f$ can be extended into an analytical function $\tilde{f}$. In this case, we can expand

$$
f(j+k)+f(j-k)=2 \sum_{\ell=0}^{\infty} \frac{k^{2 \ell}}{(2 \ell) !} \tilde{f}^{(2 \ell)}(j) .
$$

Therefore,

$$
\begin{aligned}
\mathscr{G} f(j)= & \left(p_{0}-1\right) f(j)+2 \sum_{k=1}^{N} p_{k} \sum_{\ell=0}^{\infty} \frac{k^{2 \ell}}{(2 \ell) !} \widetilde{f}^{(2 \ell)}(j) \\
= & \left(p_{0}+2 \sum_{k=1}^{N} p_{k}-1\right) f(j) \\
& +2 \sum_{\ell=1}^{\infty}\left(\sum_{k=1}^{N} k^{2 \ell} p_{k}\right) \frac{\widetilde{f}^{(2 \ell)}(j)}{(2 \ell) !} .
\end{aligned}
$$

Since $p_{0}+2 \sum_{k=1}^{N} p_{k}=1$, we see that the expression (5) of $\mathscr{G}$ holds if and only if the $p_{k}$ 's satisfy the equations

$$
\begin{gathered}
\sum_{k=1}^{N} k^{2 \ell} p_{k}=0 \quad \text { for } 1 \leq \ell \leq N-1, \\
\sum_{k=1}^{N} k^{2 N} p_{k}=\frac{1}{2} \kappa_{N} c(2 N) ! .
\end{gathered}
$$

Proposition 1. The numbers $p_{k}, k \in\{1, \ldots, N\}$, satisfying (19), are given by

$$
p_{k}=(-1)^{k-1} c\left(\begin{array}{c}
2 N \\
k+N
\end{array}\right) .
$$

In particular, $p_{N}=\kappa_{N} c$.

Proof. First, we recall that the solution of a Vandermonde system of the form $\sum_{k=1}^{N} a_{k}^{\ell} x_{k}=\alpha_{\ell}, 1 \leq \ell \leq N$, is given by

$$
x_{k}=\frac{A_{k}\left(\begin{array}{c}
a_{1}, \ldots, a_{N} \\
\alpha_{1}, \ldots, \alpha_{N}
\end{array}\right)}{A\left(a_{1}, \ldots, a_{N}\right)}, \quad 1 \leq k \leq N
$$

with

$$
A\left(a_{1}, \ldots, a_{N}\right)=\left|\begin{array}{ccc}
a_{1} & \cdots & a_{N} \\
a_{1}^{2} & \cdots & a_{N}^{2} \\
\vdots & & \vdots \\
a_{1}^{N} & \cdots & a_{N}^{N}
\end{array}\right|
$$

and, for any $k \in\{1, \ldots, N\}$,

$$
A_{k}\left(\begin{array}{c}
a_{1}, \ldots, a_{N} \\
\alpha_{1}, \ldots, \alpha_{N}
\end{array}\right)=\left|\begin{array}{ccccccc}
a_{1} & \cdots & a_{k-1} & \alpha_{1} & a_{k+1} & \cdots & a_{N} \\
a_{1}^{2} & \cdots & a_{k-1}^{2} & \alpha_{2} & a_{k+1}^{2} & \cdots & a_{N}^{2} \\
\vdots & & \vdots & \vdots & \vdots & & \vdots \\
a_{1}^{N} & \cdots & a_{k-1}^{N} & \alpha_{N} & a_{k+1}^{N} & \cdots & a_{N}^{N}
\end{array}\right|
$$

In the notation of $A_{k}$ and that of forthcoming determinants, we adopt the convention that when the index of certain entries in the determinant lies out of the range of $k$, the corresponding column is discarded. That is, for $k=1$ and $k=N$, the respective determinants write

$$
\begin{aligned}
A_{1}\left(\begin{array}{c}
a_{1}, \ldots, a_{N} \\
\alpha_{1}, \ldots, \alpha_{N}
\end{array}\right) & =\left|\begin{array}{cccc}
\alpha_{1} & a_{2} & \cdots & a_{N} \\
\vdots & \vdots & & \vdots \\
\alpha_{N} & a_{2}^{N} & \cdots & a_{N}^{N}
\end{array}\right|, \\
A_{N}\left(\begin{array}{c}
a_{1}, \ldots, a_{N} \\
\alpha_{1}, \ldots, \alpha_{N}
\end{array}\right) & =\left|\begin{array}{cccc}
a_{1} & \cdots & a_{N-1} & \alpha_{1} \\
\vdots & & \vdots & \vdots \\
a_{1}^{N} & \cdots & a_{N-1}^{N} & \alpha_{N}
\end{array}\right| .
\end{aligned}
$$


It is well-known that, for any $k \in\{1, \ldots, N\}$,

$$
\begin{aligned}
A\left(a_{1}, \ldots, a_{N}\right)= & \prod_{1 \leq j \leq N} a_{j} \prod_{1 \leq \ell<m \leq N}\left(a_{m}-a_{\ell}\right) \\
= & (-1)^{k+N} \prod_{\substack{1 \leq j \leq N \\
a_{j}}}\left(a_{\substack{1 \leq j \leq N \\
j \neq k}}-a_{j}\right) \\
& \times \prod_{\substack{1 \leq \ell<m \leq N \\
\ell, m \neq k}}\left(a_{m}-a_{\ell}\right) .
\end{aligned}
$$

In the particular case where $\alpha_{\ell}=0$ for $1 \leq \ell \leq N-1$, we have, for any $k \in\{1, \ldots, N\}$, that

$$
\begin{aligned}
A_{k}\left(\begin{array}{c}
a_{1}, \ldots, a_{N} \\
\alpha_{1}, \ldots, \alpha_{N}
\end{array}\right)= & (-1)^{k+N} \alpha_{N} \\
& \times\left|\begin{array}{cccccc}
a_{1} & \cdots & a_{k-1} & a_{k+1} & \cdots & a_{N} \\
a_{1}^{2} & \cdots & a_{k-1}^{2} & a_{k+1}^{2} & \cdots & a_{N}^{2} \\
\vdots & & \vdots & \vdots & & \vdots \\
a_{1}^{N-1} & \cdots & a_{k-1}^{N-1} & a_{k+1}^{N-1} & \cdots & a_{N}^{N-1}
\end{array}\right| . \\
= & (-1)^{k+N} \alpha_{N} \prod_{\substack{1 \leq j \leq N \\
j \neq k}} a_{j} \prod_{\substack{1 \leq \ell<m \leq N \\
\ell, m \neq k}}\left(a_{m}-a_{\ell}\right) .
\end{aligned}
$$

Therefore, the solution simply writes

$$
x_{k}=\frac{\alpha_{N}}{a_{k} \prod_{\substack{1 \leq j \leq N \\ j \neq k}}\left(a_{k}-a_{j}\right)}, \quad 1 \leq k \leq N .
$$

Now, we see that system (19) is a Vandermonde system with the choices $a_{k}=k^{2}, x_{k}=p_{k}$, and $\alpha_{\ell}=0$ for $1 \leq \ell \leq$ $N-1, \alpha_{N}=\kappa_{N} c(2 N) ! / 2$. With these settings at hands, we explicitly have

$$
\begin{aligned}
a_{k} \prod_{\substack{1 \leq j \leq N \\
j \neq k}}\left(a_{k}-a_{j}\right) & =k^{2} \prod_{\substack{1 \leq j \leq N \\
j \neq k}}\left(k^{2}-j^{2}\right) \\
& =k^{2} \prod_{\substack{1 \leq j \leq N \\
j \neq k}}(k-j) \prod_{\substack{1 \leq j \leq N \\
j \neq k}}(k+j) \\
& =\frac{1}{2}(-1)^{N-k}(N+k) !(N-k) !
\end{aligned}
$$

and the result of Proposition 1 ensues.

Finally, the value of $p_{0}$ is obtained as follows: by using the fact that $\sum_{k=0}^{2 N}(-1)^{k}\left(\begin{array}{c}2 N \\ k\end{array}\right)=0$,

$$
\begin{aligned}
p_{0} & =1-\sum_{\substack{-N \leq k \leq N \\
k \neq 0}} p_{k} \\
& =1+c \sum_{\substack{-N \leq k \leq N \\
k \neq 0}}(-1)^{k}\left(\begin{array}{c}
2 N \\
k+N
\end{array}\right) \\
& =1-c\left(\begin{array}{c}
2 N \\
N
\end{array}\right)+(-1)^{N} c \sum_{k=0}^{2 N}(-1)^{k}\left(\begin{array}{c}
2 N \\
k
\end{array}\right) \\
& =1-c\left(\begin{array}{c}
2 N \\
N
\end{array}\right) .
\end{aligned}
$$

We find it interesting to compute the cumulative sums of the $p_{j}$ 's: for $k \in\{-N, \ldots, N\}$,

$$
\begin{aligned}
\sum_{j=-N}^{k} p_{j} & =\sum_{j=-N}^{k}\left[\delta_{j 0}+(-1)^{j-1} c\left(\begin{array}{c}
2 N \\
j+N
\end{array}\right)\right] \\
& =\mathbb{1}_{\{k \geq 0\}}+(-1)^{N-1} c \sum_{j=0}^{k+N}(-1)^{j}\left(\begin{array}{c}
2 N \\
j
\end{array}\right) .
\end{aligned}
$$

The last displayed sum is classical and easy to compute by appealing to Pascal's formula which leads to a telescopic sum:

$$
\begin{aligned}
\sum_{j=0}^{k+N}(-1)^{j}\left(\begin{array}{c}
2 N \\
j
\end{array}\right) & \\
& =\sum_{j=0}^{k+N}\left[(-1)^{j}\left(\begin{array}{c}
2 N-1 \\
j-1
\end{array}\right)-(-1)^{j+1}\left(\begin{array}{c}
2 N-1 \\
j
\end{array}\right)\right] \\
& =(-1)^{k+N}\left(\begin{array}{c}
2 N-1 \\
k+N
\end{array}\right) .
\end{aligned}
$$

Thus, for $k \in\{-N, \ldots, N\}$,

$$
\sum_{j=-N}^{k} p_{j}=\mathbb{1}_{\{k \geq 0\}}+(-1)^{k-1} c\left(\begin{array}{c}
2 N-1 \\
k+N
\end{array}\right) .
$$

Observe that this sum is nothing but $\mathbb{P}\left\{\xi_{1} \leq k\right\}$. Next, we compute the total sum of the $\left|p_{j}\right|$ 's: by using the fact that $\sum_{k=0}^{2 N}\left(\begin{array}{c}2 N \\ k\end{array}\right)=4^{N}$,

$$
\begin{aligned}
\sum_{k=-N}^{N}\left|p_{k}\right| & =\left|1-c\left(\begin{array}{c}
2 N \\
N
\end{array}\right)\right|+\sum_{\substack{-N \leq k \leq N \\
k \neq 0}} c\left(\begin{array}{c}
2 N \\
k+N
\end{array}\right) \\
& =\left|1-c\left(\begin{array}{c}
2 N \\
N
\end{array}\right)\right|+c\left[4^{N}-\left(\begin{array}{c}
2 N \\
N
\end{array}\right)\right] \\
& =c 4^{N}-1+2\left[1-c\left(\begin{array}{c}
2 N \\
N
\end{array}\right)\right]^{+}
\end{aligned}
$$

As previously mentioned, there is an interpretation to this sum: this is the total variation of the pseudodistribution of $\xi_{1}$. We can also explicitly determine the generating function of $\xi_{1}$ : for any $\zeta \in \mathbb{C}^{*}$,

$$
\begin{aligned}
\mathbb{E}\left(\zeta^{\xi_{1}}\right) & =\sum_{k=-N}^{N} p_{k} \zeta^{k} \\
& =1+c\left[\sum_{k=-N}^{N}(-1)^{k-1}\left(\begin{array}{c}
2 N \\
k+N
\end{array}\right) \zeta^{k}\right] \\
& =1+(-1)^{N-1} \frac{c}{\zeta^{N}}\left[\sum_{k=0}^{2 N}(-1)^{k}\left(\begin{array}{c}
2 N \\
k
\end{array}\right) \zeta^{k}\right] \\
& =1+\kappa_{N} c \frac{(1-\zeta)^{2 N}}{\zeta^{N}} .
\end{aligned}
$$

We sum up below the results we have obtained concerning the pseudodistribution of $\xi_{1}$. 
Proposition 2. The pseudodistribution of $\xi_{1}$ is determined, for $k \in\{-N, \ldots, N\}$, by

$$
\mathbb{P}\left\{\xi_{1}=k\right\}=\delta_{k 0}+(-1)^{k-1} c\left(\begin{array}{c}
2 N \\
k+N
\end{array}\right)
$$

or, equivalently, by

$$
\mathbb{P}\left\{\xi_{1} \leq k\right\}=\mathbb{1}_{\{k \geq 0\}}+(-1)^{k-1} c\left(\begin{array}{c}
2 N-1 \\
k+N
\end{array}\right) .
$$

The total variation of the pseudodistribution of $\xi_{1}$ is given by

$$
\left\|\mathbb{P}_{\xi_{1}}\right\|= \begin{cases}1+c\left[4^{N}-2\left(\begin{array}{c}
2 N \\
N
\end{array}\right)\right] & \text { if } 0<c \leq \frac{1}{\left(\begin{array}{c}
2 N \\
N
\end{array}\right)} \\
c 4^{N}-1 & \text { if } c \geq \frac{1}{\left(\begin{array}{c}
2 N \\
N
\end{array}\right)}\end{cases}
$$

The generating function of $\xi_{1}$ is given, for any $\zeta \in \mathbb{C}^{*}$, by

$$
\mathbb{E}\left(\zeta^{\xi_{1}}\right)=1+\kappa_{N} c \frac{(1-\zeta)^{2 N}}{\zeta^{N}}
$$

In particular, the Fourier transform of $\xi_{1}$ admits the following expression: for any $\theta \in[0,2 \pi]$, by

$$
\mathbb{E}\left(\mathrm{e}^{i \theta \xi_{1}}\right)=1-c 4^{N} \sin ^{2 N}\left(\frac{\theta}{2}\right)
$$

Remark 3. For $c=1 /\left(\begin{array}{c}2 N \\ N\end{array}\right)$, we have $\mathbb{P}\left\{\xi_{1}=0\right\}=0$; that is, the pseudorandom walk does not stay at its current location. If $0<c \leq 1 /\left(\begin{array}{c}2 N+1 \\ N+1\end{array}\right)$, it can be easily seen, by using the identity $\left(\begin{array}{c}2 N \\ N\end{array}\right)+\left(\begin{array}{c}2 N \\ N+1\end{array}\right)=\left(\begin{array}{c}2 N+1 \\ N+1\end{array}\right)$, that $\mathbb{P}\left\{\xi_{1}=0\right\} \geq \mathbb{P}\left\{\xi_{1}=1\right\}>0$. On the other hand, for any $c>0$, it is clear that $\mathbb{P}\left\{\xi_{1}=1\right\}>$ $\left|\mathbb{P}\left\{\xi_{1}=2\right\}\right|>\cdots>\left|\mathbb{P}\left\{\xi_{1}=N\right\}\right|$. In Table 1 and Figures 1 and 2 , we provide some numerical values and (rescaled) profiles of the pseudodistribution of $\xi_{1}$ for $N=3$ and $N=4$ and several values of $c$.

In the sequel, we will use the total variation of $\xi_{1}$ as an upper bound which we call $M_{1}$ :

$$
M_{1}= \begin{cases}1+c\left[4^{N}-2\left(\begin{array}{c}
2 N \\
N
\end{array}\right)\right] & \text { if } 0<c \leq \frac{1}{\left(\begin{array}{c}
2 N \\
N
\end{array}\right)}, \\
c 4^{N}-1 & \text { if } c \geq \frac{1}{\left(\begin{array}{c}
2 N \\
N
\end{array}\right)} .\end{cases}
$$

Set $\mathfrak{f}(\theta)=\mathbb{E}\left(e^{i \theta \xi_{1}}\right)$ for any $\theta \in[0,2 \pi]$. We notice that $\mathfrak{f}(\theta) \in$ $\left[1-c 4^{N}, 1\right]$ and, more precisely,

$$
\begin{aligned}
\|\tilde{f}\|_{\infty} & =\sup _{\theta \in[0,2 \pi]}|\mathfrak{f}(\theta)| \\
& =\max \left(\left|1-c 4^{N}\right|, 1\right) \\
& = \begin{cases}1 & \text { if } 0<c \leq \frac{1}{2^{2 N-1}}, \\
c 4^{N}-1 & \text { if } c \geq \frac{1}{2^{2 N-1}} .\end{cases}
\end{aligned}
$$

TABLE 1: Some numerical values in the cases $N \in\{3,4\}$.

(a)

\begin{tabular}{lcccc}
\hline$c$ & $p_{o}$ & $p_{1}$ & $p_{2}$ & $p_{3}$ \\
\hline 1 & -19 & 15 & -6 & 1 \\
$1 / 20$ & 0 & 0.75 & -0.30 & 0.050 \\
$1 / 32$ & 0.38 & 0.47 & -0.19 & 0.031 \\
$1 / 35$ & 0.43 & 0.43 & -0.17 & 0.029 \\
$1 / 64$ & 0.69 & 0.23 & -0.094 & 0.016 \\
\hline
\end{tabular}

(b)

\begin{tabular}{lccccc}
\hline$c$ & $p_{o}$ & $p_{1}$ & $p_{2}$ & $p_{3}$ & $p_{4}$ \\
\hline 1 & -69 & 56 & -28 & 8 & -1 \\
$1 / 70$ & 0 & 0.8 & -0.4 & 0.114 & -0.0143 \\
$1 / 126$ & 0.44 & 0.44 & -0.22 & 0.063 & -0.0079 \\
$1 / 128$ & 0.45 & 0.44 & -0.22 & 0.062 & -0.0078 \\
$1 / 256$ & 0.73 & 0.22 & -0.11 & 0.031 & -0.0039 \\
\hline
\end{tabular}

Let us denote this bound by $M_{\infty}$ :

$$
M_{\infty}= \begin{cases}1 & \text { if } 0<c \leq \frac{1}{2^{2 N-1}} \\ c 4^{N}-1 & \text { if } c \geq \frac{1}{2^{2 N-1}} .\end{cases}
$$

In view of (40) and (42), since $\left(\begin{array}{c}2 N \\ N\end{array}\right) \leq 2^{2 N-1}$, we see that $M_{1} \geq M_{\infty} \geq 1$.

Proposition 4. The pseudodistribution of $S_{n}$ is given, for any $k \in\{-N n, \ldots, N n\}$, by

$$
\mathbb{P}\left\{S_{n}=k\right\}=(-1)^{k} \sum_{\ell=0}^{n}(-c)^{\ell}\left(\begin{array}{l}
n \\
\ell
\end{array}\right)\left(\begin{array}{c}
2 N \ell \\
k+N \ell
\end{array}\right) .
$$

Actually, the foregoing sum is taken over the $\ell$ such that $\ell \geq$ $|k| / N$. We also have that

$$
\mathbb{P}\left\{S_{n} \leq k\right\}=\mathbb{1}_{\{k \geq 0\}}+(-1)^{k} \sum_{\ell=1}^{n}(-c)^{\ell}\left(\begin{array}{l}
n \\
\ell
\end{array}\right)\left(\begin{array}{c}
2 N \ell-1 \\
k+N \ell
\end{array}\right) .
$$

Proof. By the independence of the $\xi_{j}$ 's which have the same pseudoprobability distribution, we plainly have that

$$
\mathbb{E}\left(\mathrm{e}^{i \theta S_{n}}\right)=\mathfrak{f}(\theta)^{n}=\left[1-c 4^{N} \sin ^{2 N}\left(\frac{\theta}{2}\right)\right]^{n} .
$$

Hence, by inverse Fourier transform, we extract that

$$
\begin{aligned}
\mathbb{P}\left\{S_{n}=k\right\} & =\frac{1}{2 \pi} \int_{0}^{2 \pi} \tilde{\mathrm{f}}(\theta)^{n} \mathrm{e}^{-i k \theta} \mathrm{d} \theta \\
& =\sum_{\ell=0}^{n}\left(-4^{N} c\right)^{\ell}\left(\begin{array}{l}
n \\
\ell
\end{array}\right) \frac{1}{2 \pi} \int_{0}^{2 \pi} \sin ^{2 N \ell}\left(\frac{\theta}{2}\right) \mathrm{e}^{-i k \theta} \mathrm{d} \theta .
\end{aligned}
$$



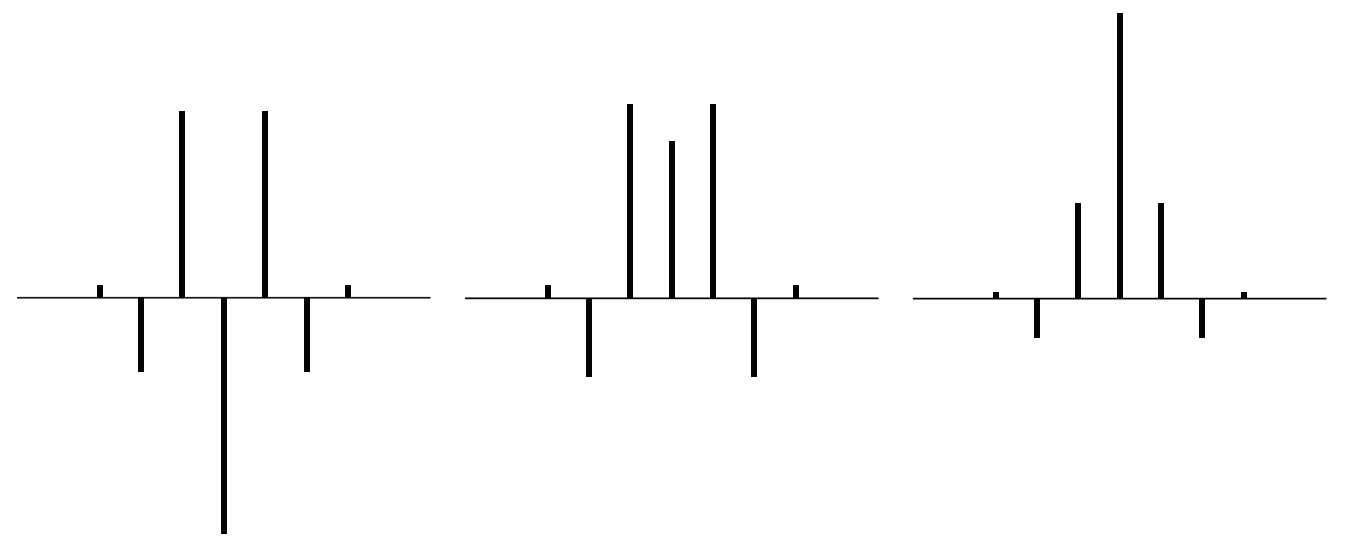

Figure 1: $N=3, c \in\{1,1 / 32,1 / 64\}$.
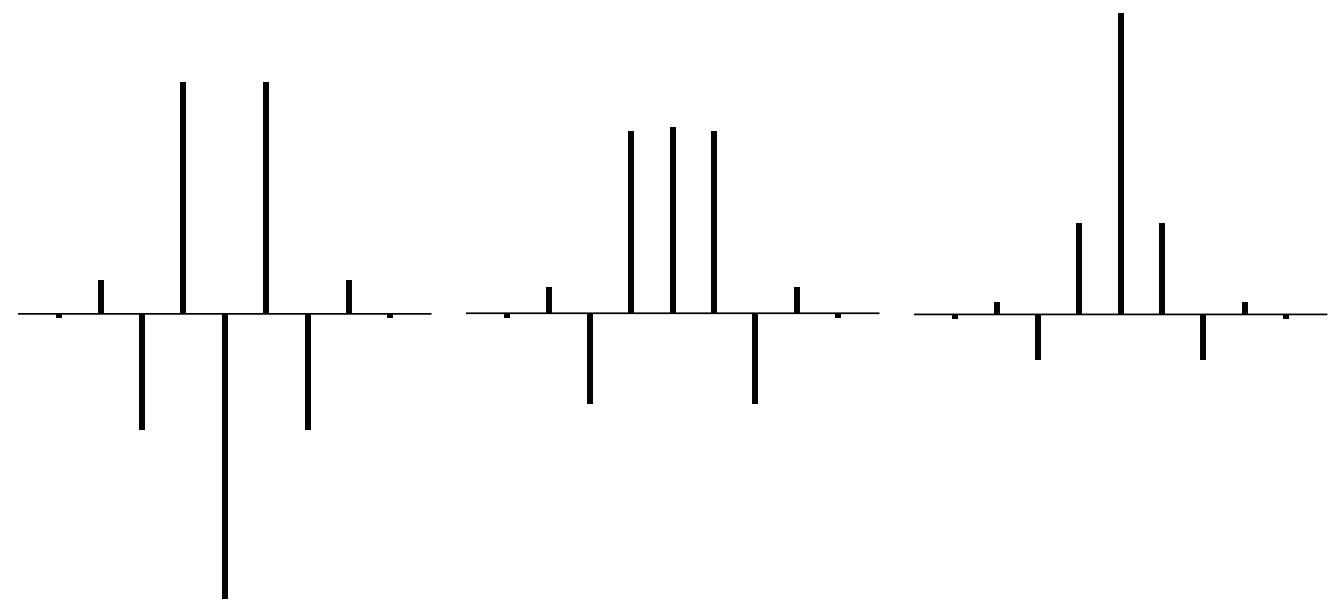

Figure 2: $N=4, c \in\{1,1 / 128,1 / 256\}$.

By writing $\sin (\theta / 2)=\left(\mathrm{e}^{i \theta / 2}-\mathrm{e}^{-i \theta / 2}\right) /(2 i)$, we get for the integral lying in (47) that

$$
\begin{aligned}
& \frac{1}{2 \pi} \int_{0}^{2 \pi} \sin ^{2 N \ell}\left(\frac{\theta}{2}\right) \mathrm{e}^{-i k \theta} \mathrm{d} \theta \\
& =\frac{1}{2 \pi} \int_{0}^{2 \pi}\left(\frac{\mathrm{e}^{i \theta / 2}-\mathrm{e}^{-i \theta / 2}}{2 i}\right)^{2 N \ell} \mathrm{e}^{-i k \theta} \mathrm{d} \theta \\
& =\frac{(-1)^{N \ell}}{(2 \pi) 4^{N \ell}} \int_{0}^{2 \pi} \sum_{m=0}^{2 N \ell}(-1)^{m}\left(\begin{array}{c}
2 N \ell \\
m
\end{array}\right) \mathrm{e}^{i(m-k-N \ell) \theta} \mathrm{d} \theta \\
& =\frac{(-1)^{N \ell}}{4^{N \ell}} \sum_{m=0}^{2 N \ell}(-1)^{m}\left(\begin{array}{c}
2 N \ell \\
m
\end{array}\right) \frac{1}{2 \pi} \int_{0}^{2 \pi} \mathrm{e}^{i(m-k-N \ell) \theta} \mathrm{d} \theta \\
& =\frac{(-1)^{k}}{4^{N \ell}}\left(\begin{array}{c}
2 N \ell \\
k+N \ell
\end{array}\right) .
\end{aligned}
$$

By plugging (48) into (47), we derive (43). Next, we write, for $k \in\{-N n, \ldots, N n\}$, that

$$
\begin{aligned}
\mathbb{P}\left\{S_{n} \leq k\right\} & =\sum_{j=-N n}^{k} \mathbb{P}\left\{S_{n}=j\right\} \\
& =\sum_{\ell=0}^{n}(-c)^{\ell}\left(\begin{array}{l}
n \\
\ell
\end{array}\right) \sum_{j=(-N n) \vee(-N \ell)}^{k \wedge(N \ell)}(-1)^{j}\left(\begin{array}{c}
2 N \ell \\
j+N \ell
\end{array}\right) \\
& =\sum_{\ell=0}^{n}(-c)^{\ell}\left(\begin{array}{l}
n \\
\ell
\end{array}\right) \sum_{j=-N \ell}^{k \wedge(N \ell)}(-1)^{j}\left(\begin{array}{c}
2 N \ell \\
j+N \ell
\end{array}\right) .
\end{aligned}
$$

If $k<0$, then the term in sum (49) corresponding to $\ell=0$ vanishes and

$$
\mathbb{P}\left\{S_{n} \leq k\right\}=\sum_{\ell=1}^{n}(-c)^{\ell}\left(\begin{array}{l}
n \\
\ell
\end{array}\right) \sum_{j=-N \ell}^{k}(-1)^{j}\left(\begin{array}{c}
2 N \ell \\
j+N \ell
\end{array}\right) .
$$


The second sum in the foregoing equality is easy to compute:

$$
\begin{aligned}
& \sum_{j=-N \ell}^{k}(-1)^{j}\left(\begin{array}{c}
2 N \ell \\
j+N \ell
\end{array}\right) \\
& \quad=\sum_{j=-N \ell}^{k}\left[(-1)^{j}\left(\begin{array}{c}
2 N \ell-1 \\
j+N \ell-1
\end{array}\right)-(-1)^{j+1}\left(\begin{array}{c}
2 N \ell-1 \\
j+N \ell
\end{array}\right)\right] \\
& =(-1)^{k}\left(\begin{array}{c}
2 N \ell-1 \\
k+N \ell
\end{array}\right) .
\end{aligned}
$$

If $k \geq 0$, then the term in sum (49) corresponding to $\ell=0$ is 1 and

$$
\mathbb{P}\left\{S_{n} \leq k\right\}=1+\sum_{\ell=1}^{n}(-c)^{\ell}\left(\begin{array}{l}
n \\
\ell
\end{array}\right) \sum_{j=-N \ell}^{k \wedge(N \ell)}(-1)^{j}\left(\begin{array}{c}
2 N \ell \\
j+N \ell
\end{array}\right) .
$$

By using the convention that $\left(\begin{array}{l}\alpha \\ \beta\end{array}\right)=0$ if $\beta>\alpha$, we see that the second sum above also coincides with (51). Formula (44) ensues in both cases.

Proposition 5. The upper bound below holds true: for any positive integer $n$ and any integer $k$,

$$
\left|\mathbb{P}\left\{S_{n}=k\right\}\right| \leq \sqrt{\mathbb{P}\left\{S_{2 n}=0\right\}} \leq M_{\infty}^{n}
$$

Assume that $0<c \leq 1 / 2^{2 N-1}$. The asymptotics below holds true: for any $\delta \in(0,1 /(2 N))$,

$$
\mathbb{P}\left\{S_{n}=0\right\} \underset{n \rightarrow+\infty}{=} \mathcal{O}\left(\frac{1}{n^{\delta}}\right) .
$$

Proof. Let us introduce the usual norms of any suitable function $\phi$ :

$$
\begin{gathered}
\|\phi\|_{1}=\frac{1}{2 \pi} \int_{0}^{2 \pi}|\phi(\theta)| \mathrm{d} \theta, \\
\|\phi\|_{2}=\sqrt{\frac{1}{2 \pi} \int_{0}^{2 \pi}|\phi(\theta)|^{2} \mathrm{~d} \theta} \\
\|\phi\|_{\infty}=\sup _{\theta \in[0,2 \pi]}|\phi(\theta)|
\end{gathered}
$$

and recall the elementary inequalities $\|\phi\|_{1} \leq\|\phi\|_{2} \leq\|\phi\|_{\infty}$.

It is clear from (46) that, for any integer $k$,

$$
\begin{aligned}
\left|\mathbb{P}\left\{S_{n}=k\right\}\right| & \leq \frac{1}{2 \pi} \int_{0}^{2 \pi}|\mathfrak{f}(\theta)|^{n} \mathrm{~d} \theta=\left\|\mathfrak{f}^{n}\right\|_{1} \\
& \leq\left\|\mathfrak{f}^{n}\right\|_{2}=\sqrt{\frac{1}{2 \pi} \int_{0}^{2 \pi} \mathfrak{f}(\theta)^{2 n} \mathrm{~d} \theta}=\sqrt{\mathbb{P}\left\{S_{2 n}=0\right\}} \\
& \leq\|\mathfrak{f}\|_{\infty}^{n}=M_{\infty}^{n} .
\end{aligned}
$$

This proves (53). Next, by $(46)$, since $\mathfrak{f}(2 \pi-\theta)=\mathfrak{f}(\theta)$, we have, for any $\varepsilon \in(0, \pi)$, that

$$
\begin{aligned}
\mathbb{P}\left\{S_{n}=0\right\} & =\frac{1}{2 \pi} \int_{0}^{2 \pi} \mathfrak{f}(\theta)^{n} \mathrm{~d} \theta=\frac{1}{\pi} \int_{0}^{\pi} \mathfrak{f}(\theta)^{n} \mathrm{~d} \theta \\
& =\frac{1}{\pi}\left(\int_{0}^{\varepsilon} \mathfrak{f}(\theta)^{n} \mathrm{~d} \theta+\int_{\varepsilon}^{\pi} \mathfrak{f}(\theta)^{n} \mathrm{~d} \theta\right) .
\end{aligned}
$$

The assumption $0<c<1 / 2^{2 N-1}$ entails that $|\mathfrak{f}(\theta)|<1$ for any $\theta \in(0, \pi)$. We see that $|\mathfrak{f}(\theta)| \leq 1$ on $[0, \varepsilon]$, and $|\mathfrak{f}(\theta)| \leq|\mathfrak{f}(\varepsilon)|$ on $[\varepsilon, \pi]$ for any $\varepsilon \in(0, \pi)$. Hence,

$$
\left|\mathbb{P}\left\{S_{n}=0\right\}\right| \leq \varepsilon+|\mathfrak{f}(\varepsilon)|^{n} .
$$

Now, choose $\varepsilon=1 / n^{\delta}$ for a positive $\delta$. We have that

$$
\ln \left(|\mathfrak{f}(\varepsilon)|^{n}\right)=n \ln \left(1-c 4^{N} \sin ^{2 N}\left(\frac{1}{2 n^{\delta}}\right)\right) \sim-c n^{1-2 N \delta}
$$

which clearly entails, for large enough $n$, that $|\mathfrak{f}(\varepsilon)|^{n} \leq$ $\exp \left(-(c / 2) n^{1-2 N \delta}\right)$. Thus, if $\delta \in(0,1 /(2 N)),|\mathfrak{f}(\varepsilon)|^{n}=o(\varepsilon)$ which proves (54).

If $c=1 / 2^{2 N-1}, \mathfrak{f}(\theta)=1-2 \sin ^{2 N}(\theta / 2)$. In this case, the same holds true upon splitting the integral $\int_{0}^{\pi}$ into $\int_{0}^{\varepsilon}+\int_{\varepsilon}^{\pi-\varepsilon}+\int_{\pi-\varepsilon}^{\pi}$.

Remark 6. A better estimate for $\left|\mathbb{P}\left\{S_{n}=k\right\}\right|$ can be obtained in the same way:

$$
\forall k \in \mathbb{Z}, \quad\left|\mathbb{P}\left\{S_{n}=k\right\}\right| \leq \begin{cases}\mathbb{P}\left\{S_{n}=0\right\} & \text { if } n \text { is even, } \\ M_{\infty} \mathbb{P}\left\{S_{n-1}=0\right\} & \text { if } n \text { is odd. }\end{cases}
$$

Nevertheless, we will not use it. We also have the following inequality for the total variation of $S_{n}$ :

$$
\left\|\mathbb{P}_{S_{n}}\right\|=\sum_{k=-N n}^{N n}\left|\mathbb{P}\left\{S_{n}=k\right\}\right| \leq\left\|\mathbb{P}_{\xi_{1}}\right\|^{n}=M_{1}^{n} .
$$

Proposition 7. For any bounded function $F$ defined on $\mathbb{Z}^{n}$,

$$
\left|\mathbb{E}\left[F\left(S_{1}, \ldots, S_{n}\right)\right]\right| \leq\|F\|_{\infty} M_{1}^{n} .
$$

Proof. Recall that we set $p_{k}=\mathbb{P}\left\{\xi_{1}=k\right\}$ for any $k \epsilon$ $\{-N, \ldots, N\}$. We extend these settings by putting $p_{k}=0$ for $k \in \mathbb{Z} \backslash\{-N, \ldots, N\}$. We have that

$$
\begin{aligned}
\mid \mathbb{E} & {\left[F\left(S_{1}, \ldots, S_{n}\right)\right] \mid } \\
& =\left|\sum_{\left(k_{1}, \ldots, k_{n}\right) \in \mathbb{Z}^{n}} F\left(k_{1}, \ldots, k_{n}\right) \mathbb{P}\left\{S_{1}=k_{1}, \ldots, S_{n}=k_{n}\right\}\right| \\
& \leq\|F\|_{\infty} \sum_{\left(k_{1}, \ldots, k_{n}\right) \in \mathbb{Z}^{n}}\left|p_{k_{1}} p_{k_{2}-k_{1}} \cdots p_{k_{n}-k_{n-1}}\right| .
\end{aligned}
$$


The foregoing sum can be easily evaluated as follows:

$$
\begin{aligned}
& \sum_{\left(k_{1}, \ldots, k_{n}\right) \in \mathbb{Z}^{n}}\left|p_{k_{1}} p_{k_{2}-k_{1}} \cdots p_{k_{n}-k_{n-1}}\right| \\
= & \sum_{k_{1} \in \mathbb{Z}}\left|p_{k_{1}}\right|\left(\sum_{k_{2} \in \mathbb{Z}}\left|p_{k_{2}-k_{1}}\right|\left(\cdots\left(\sum_{k_{n} \in \mathbb{Z}}\left|p_{k_{n}-k_{n-1}}\right|\right) \cdots\right)\right) \\
= & \left(\sum_{k \in \mathbb{Z}}\left|p_{k}\right|\right)^{n}=M_{1}^{n}
\end{aligned}
$$

which proves (62).

2.2. Generating Function of $S_{n}$. Let us introduce the generating functions, defined for complex numbers $z, \zeta$, by

$$
\begin{gathered}
G_{k}(z)=\sum_{n \in \mathbb{N}} \mathbb{P}\left\{S_{n}=k\right\} z^{n}=\sum_{\substack{n \in \mathbb{N}: \\
n \geq|k| / N}} \mathbb{P}\left\{S_{n}=k\right\} z^{n} \text { for } k \in \mathbb{Z}, \\
G(z, \zeta)=\sum_{k \in \mathbb{Z}, n \in \mathbb{N}} \mathbb{P}\left\{S_{n}=k\right\} \zeta^{k} z^{n}=\sum_{\substack{k \in \mathbb{Z}, n \in \mathbb{N}: \\
|k| \leq N n}} \mathbb{P}\left\{S_{n}=k\right\} \zeta^{k} z^{n} .
\end{gathered}
$$

We first study the problem of convergence of the foregoing series. We start from

$$
\sum_{k \in \mathbb{Z}, n \in \mathbb{N}}\left|\mathbb{P}\left\{S_{n}=k\right\} \zeta^{k} z^{n}\right| \leq \sum_{\substack{k \in \mathbb{Z}, n \in \mathbb{N}: \\|k| \leq N n}}|\zeta|^{k}\left|M_{\infty} z\right|^{n} .
$$

If $\zeta \neq 0$ and $|\zeta| \neq 1$, then

$$
\begin{aligned}
& \sum_{\substack{k \in \mathbb{Z}, n \in \mathbb{N}: \\
|k| \leq N n}}|\zeta|^{k}\left|M_{\infty} z\right|^{n} \\
= & \sum_{n=0}^{\infty}\left(\frac{1-|\zeta|^{N n+1}}{1-|\zeta|}+\frac{1-1 /|\zeta|^{N n+1}}{1-1 /|\zeta|}-1\right)\left|M_{\infty} z\right|^{n} \\
= & \frac{1}{1-|\zeta|}\left(\sum_{n=0}^{\infty}\left|M_{\infty} z\right|^{n}-|\zeta| \sum_{n=0}^{\infty}\left|M_{\infty} z \zeta^{N}\right|^{n}\right) \\
& +\frac{1}{1-1 /|\zeta|}\left(\sum_{n=0}^{\infty}\left|M_{\infty} z\right|^{n}-\frac{1}{|\zeta|} \sum_{n=0}^{\infty}\left|\frac{M_{\infty} z}{\zeta^{N}}\right|^{n}\right)-\sum_{n=0}^{\infty}\left|M_{\infty} z\right|^{n}
\end{aligned}
$$

If we choose $z, \zeta$ such that $\left|M_{\infty} z\right|<1,\left|M_{\infty} z \zeta^{N}\right|<1$ and $\left|M_{\infty} z / \zeta^{N}\right|<1$ (which is equivalent to $|z|<1 / M_{\infty} \times$ $[\min (|\zeta|, 1 /|\zeta|)]^{N}$, or $\left.\sqrt[N]{\left|M_{\infty} z\right|}<|\zeta|<1 / \sqrt[N]{\left|M_{\infty} z\right|}\right)$, then the double sum defining the function $G(z, \zeta)$ is absolutely summable. If $|\zeta|=1$, then

$$
\sum_{\substack{k \in \mathbb{Z}, n \in \mathbb{N}: \\|k| \leq N n}}|\zeta|^{k}\left|M_{\infty} z\right|^{n}=\sum_{n=0}^{\infty}(2 N n+1)\left|M_{\infty} z\right|^{n}
$$

If we choose $z$ such that $\left|M_{\infty} z\right|<1$, then the same conclusion holds.

Now, we have that

$$
\begin{aligned}
G(z, \zeta) & =\sum_{n \in \mathbb{N}}\left(\sum_{k \in \mathbb{Z}} \mathbb{P}\left\{S_{n}=k\right\} \zeta^{k}\right) z^{n} \\
& =\sum_{n \in \mathbb{N}} \mathbb{E}\left(\zeta^{S_{n}}\right) z^{n}=\sum_{n \in \mathbb{N}}\left[z \mathbb{E}\left(\zeta^{\xi_{1}}\right)\right]^{n} \\
& =\frac{1}{1-z \mathbb{E}\left(\zeta^{\xi_{1}}\right)}
\end{aligned}
$$

and, thanks to (38), we can state the following result.

Proposition 8. The double generating function of the $\mathbb{P}\left\{S_{n}=\right.$ $k\}, k \in \mathbb{Z}, n \in \mathbb{N}$, is given, for any complex numbers $z$, $\zeta$ such that $\sqrt[N]{\left|M_{\infty} z\right|}<|\zeta|<1 / \sqrt[N]{\left|M_{\infty} z\right|}$, by

$$
G(z, \zeta)=\frac{\zeta^{N}}{(1-z) \zeta^{N}-\kappa_{N} c z(1-\zeta)^{2 N}}
$$

In particular, for any $\theta \in[0,2 \pi]$ and $z \in \mathbb{C}$ such that $|z|<$ $1 / M_{\infty}$,

$$
G\left(z, \mathrm{e}^{i \theta}\right)=\frac{1}{1-z+c 4^{N} z \sin ^{2 N}(\theta / 2)} .
$$

On the other hand,

$$
G(z, \zeta)=\sum_{k \in \mathbb{Z}}\left(\sum_{n \in \mathbb{N}} \mathbb{P}\left\{S_{n}=k\right\} z^{n}\right) \zeta^{k}=\sum_{k \in \mathbb{Z}} G_{k}(z) \zeta^{k} .
$$

By substituting $\zeta=\mathrm{e}^{i \theta}$ in the foregoing equality, we get the Fourier series of the function $\theta \mapsto G\left(z, \mathrm{e}^{i \theta}\right)$ :

$$
G\left(z, \mathrm{e}^{i \theta}\right)=\sum_{k \in \mathbb{Z}} G_{k}(z) \mathrm{e}^{i k \theta}
$$

from which we extract the sequence of the coefficients $\left(G_{k}(z)\right)_{k \in \mathbb{N}}$. Indeed, since $\mathbb{P}\left\{S_{n}=-k\right\}=\mathbb{P}\left\{S_{n}=k\right\}$, we have that $G_{k}(z)=G_{-k}(z)$ and

$$
\begin{aligned}
G_{k}(z) & =\frac{1}{2 \pi} \int_{0}^{2 \pi} G\left(z, \mathrm{e}^{i \theta}\right) \mathrm{e}^{-i k \theta} \mathrm{d} \theta \\
& =\frac{1}{2 \pi} \int_{0}^{2 \pi} G\left(z, \mathrm{e}^{i \theta}\right) \mathrm{e}^{i k \theta} \mathrm{d} \theta \\
& =\frac{1}{2 \pi i} \int_{\mathscr{C}} G(z, \zeta) \zeta^{k-1} \mathrm{~d} \zeta
\end{aligned}
$$

where $\mathscr{C}$ is the circle of radius 1 centered at the origin and counter clockwise orientated. Then, referring to (70), we obtain, for any $z \in \mathbb{C}$ satisfying $|z|<1 / M_{\infty}$, that

$$
G_{k}(z)=\frac{1}{2 \pi i} \int_{\mathscr{C}} \frac{\zeta^{k+N-1}}{P(z, \zeta)} \mathrm{d} \zeta
$$


where $P(z, \zeta)$ is the polynomial given by

$$
P(z, \zeta)=(1-z) \zeta^{N}-\kappa_{N} c z(\zeta-1)^{2 N}
$$

We are looking for the roots of $\zeta \mapsto P(z, \zeta)$ which lie inside the circle $\mathscr{C}$. For this, we introduce the $N$ th roots of $\kappa_{N}: \theta_{j}=$ $-\mathrm{e}^{i((2 j-1) / N) \pi}$ for $1 \leq j \leq N ; \theta_{j}^{N}=\kappa_{N}$.

From now on, in order to simplify the expression of the roots of $P$, we make the assumption that $z$ is a real number lying in $\left(0,1 / M_{\infty}\right)$ (and then $\left.z \in(0,1)\right)$. The roots of $\zeta \mapsto$ $P(z, \zeta)$ are those of the equations $\zeta^{2}-2\left[1+\theta_{j} w(z)\right] \zeta+1=0$, $1 \leq j \leq N$, where

$$
w(z)=\frac{\sqrt[N]{1-z}}{2 \sqrt[N]{c z}}
$$

They can be written as

$$
\begin{aligned}
u_{j}(z)= & 1+\theta_{j} w(z) \\
& -\theta_{j} \sqrt{w(z)}\left[a_{j}(z)+i \epsilon_{j} b_{j}(z)\right], \quad 1 \leq j \leq N, \\
v_{j}(z)= & 1+\theta_{j} w(z) \\
& +\theta_{j} \sqrt{w(z)}\left[a_{j}(z)+i \epsilon_{j} b_{j}(z)\right], \quad 1 \leq j \leq N,
\end{aligned}
$$

with

$$
\epsilon_{j}=\operatorname{sgn}\left(\sin \left(\frac{2 j-1}{N} \pi\right)\right)
$$

(with the convention that $\operatorname{sgn}(0)=0$ ),

$$
\begin{aligned}
a_{j}(z)=\frac{1}{\sqrt{2}} & {\left[\sqrt{w(z)^{2}-4 \cos \left(\frac{2 j-1}{N} \pi\right) w(z)+4}\right.} \\
& \left.+w(z)-2 \cos \left(\frac{2 j-1}{N} \pi\right)\right]^{1 / 2}, \\
b_{j}(z)=\frac{1}{\sqrt{2}} & {\left[\sqrt{w(z)^{2}-4 \cos \left(\frac{2 j-1}{N} \pi\right) w(z)+4}\right.} \\
& \left.-w(z)+2 \cos \left(\frac{2 j-1}{N} \pi\right)\right]^{1 / 2} .
\end{aligned}
$$

We notice that $a_{j}(z) b_{j}(z)=|\sin (((2 j-1) / N) \pi)|$. Because of the last coefficient 1 in the polynomial $\zeta^{2}-2\left[1+\theta_{j} w(z)\right] \zeta+1$, it is clear that the roots $u_{j}(z)$ and $v_{j}(z)$ are inverse: $v_{j}(z)=$ $1 / u_{j}(z)$.

Let us check that $\left|u_{j}(z)\right|<1<\left|v_{j}(z)\right|$ for any $j \in$ $\{1, \ldots, N\}$. Straightforward computations yield that

$$
\begin{aligned}
& \left|u_{j}(z)\right|^{2}=A_{j}(z)-B_{j}(z), \\
& \left|v_{j}(z)\right|^{2}=A_{j}(z)+B_{j}(z),
\end{aligned}
$$

where

$$
\begin{aligned}
& A_{j}(z)= w(z)^{2}+w(z) \\
& \times\left[\sqrt{w(z)^{2}-4 \cos \left(\frac{2 j-1}{N} \pi\right) w(z)+4}\right. \\
&\left.-2 \cos \left(\frac{2 j-1}{N} \pi\right)\right]+1, \\
& B_{j}(z)=2 \sqrt{w(z)}\left[\left(w(z)-\cos \left(\frac{2 j-1}{N} \pi\right)\right) a_{j}(z)\right. \\
&\left.\quad+\left|\sin \left(\frac{2 j-1}{N} \pi\right)\right| b_{j}(z)\right] .
\end{aligned}
$$

Since $v_{j}(z)=1 / u_{j}(z)$, checking that $\left|u_{j}(z)\right|<1<\left|v_{j}(z)\right|$ is equivalent to checking that $\left|u_{j}(z)\right|^{2}<\left|v_{j}(z)\right|^{2}$; that is, $B_{j}(z)>0$. If $\sin (((2 j-1) / N) \pi) \neq 0$, we have $a_{j}(z) b_{j}(z) \neq 0$, $a_{j}(z)=|\sin (((2 j-1) / N) \pi)| / b_{j}(z)$ and then

$$
\begin{aligned}
B_{j}(z)= & 2 a_{j}(z) \sqrt{w(z)}\left[w(z)-\cos \left(\frac{2 j-1}{N} \pi\right)+b_{j}(z)^{2}\right] \\
= & a_{j}(z) \sqrt{w(z)} \\
& \times\left[\sqrt{w(z)^{2}-4 \cos \left(\frac{2 j-1}{N} \pi\right) w(z)+4}+w(z)\right]
\end{aligned}
$$$$
>0 \text {. }
$$

If $\sin (((2 j-1) / N) \pi)=0$ (which happens only when $N$ is odd and $j=(N+1) / 2), a_{j}(z)=\sqrt{w(z)+2}, b_{j}(z)=0$ and then $B_{j}=2 a_{j}(z) \sqrt{w(z)}(w(z)+1)>0$.

The above discussion ensures that the roots we are looking for (i.e., those lying inside $\mathscr{C}$ ) are $u_{j}(z), 1 \leq j \leq N$; we discard the $v_{j}(z)$ 's.

Remark 9. We notice that

$$
\begin{aligned}
& \lim _{z \rightarrow 1^{-}} a_{j}(z)=\sqrt{2} \sin \left(\frac{2 j-1}{2 N} \pi\right), \\
& \lim _{z \rightarrow 1^{-}} b_{j}(z)=\epsilon_{j} \sqrt{2} \cos \left(\frac{2 j-1}{2 N} \pi\right)
\end{aligned}
$$

and then

$$
\lim _{z \rightarrow 1^{-}} \theta_{j}\left[a_{j}(z)+i \epsilon_{j} b_{j}(z)\right]=\sqrt{2} \varphi_{j}
$$

where we set $\varphi_{j}=-i \mathrm{e}^{i((2 j-1) / 2 N) \pi}$. The $\varphi_{j}, 1 \leq j \leq N$, are the $(2 N)$ th roots of $\kappa_{N}$ with positive real part: $\varphi_{j}^{2 N}=\kappa_{N}$ and $\mathfrak{R}\left(\varphi_{j}\right)>0$. As a result, we derive the asymptotics, which will be used further,

$$
u_{j}(z)=1+\varepsilon_{j}(z)
$$

with $\varepsilon_{j}(z) \underset{z \rightarrow 1^{-}}{\sim}-\frac{\varphi_{j}}{\sqrt[2 N]{c}} \sqrt[2 N]{1-z}=\mathcal{O}(\sqrt[2 N]{1-z})$. 
Example 10. For $N=1$, the roots explicitly write as

$$
u_{1}(z)=1+w(z)-a_{1}(z) \sqrt{w(z)}, \quad v_{1}(z)=\frac{1}{u_{1}(z)}
$$

with

$$
w(z)=\frac{1-z}{2 c z}, \quad a_{1}(z)=\sqrt{w(z)+2} .
$$

If $c=1 / 2$, it can be simplified into

$$
u_{1}(z)=\frac{1-\sqrt{1-z^{2}}}{z}
$$

For $N=2$, the roots explicitly write as

$$
\begin{aligned}
& u_{1}(z)=\left[1-b_{1}(z) \sqrt{w(z)}\right]-i\left[w(z)-a_{1}(z) \sqrt{w(z)}\right] \\
& u_{2}(z)=\overline{u_{1}(z)}, \quad v_{1}(z)=\frac{1}{u_{1}(z)}, \quad v_{2}(z)=\overline{v_{1}(z)}
\end{aligned}
$$

with

$$
\begin{gathered}
w(z)=\frac{\sqrt{1-z}}{2 \sqrt{c z}}, \quad a_{1}(z)=\frac{1}{\sqrt{2}} \sqrt{\sqrt{w(z)^{2}+4}+w(z)} \\
b_{1}(z)=\frac{1}{\sqrt{2}} \sqrt{\sqrt{w(z)^{2}+4}-w(z)}
\end{gathered}
$$

For $N=3$, the roots explicitly write as

$$
\begin{aligned}
u_{1}(z)= & \frac{1}{2}\left[2-w(z)-\left(a_{1}(z)-b_{1}(z) \sqrt{3}\right) \sqrt{w(z)}\right] \\
& -\frac{i}{2}\left[w(z) \sqrt{3}-\left(a_{1}(z) \sqrt{3}+b_{1}(z)\right) \sqrt{w(z)}\right], \\
u_{2}(z)= & 1-w(z)-a_{2}(z) \sqrt{w(z)}, \quad u_{3}(z)=\overline{u_{1}(z)}, \\
v_{1}(z)= & \frac{1}{u_{1}(z)}, \quad v_{2}(z)=\frac{1}{u_{2}(z)}, \quad v_{3}(z)=\overline{v_{1}(z)}
\end{aligned}
$$

with

$$
\begin{gathered}
a_{1}(z)=\frac{1}{\sqrt{2}} \sqrt{\sqrt{w(z)^{2}-2 w(z)+4}+w(z)-1}, \\
b_{1}(z)=\frac{1}{\sqrt{2}} \sqrt{\sqrt{w(z)^{2}-2 w(z)+4}-w(z)+1}, \\
a_{2}(z)=\sqrt{w(z)+2}, \quad w(z)=\frac{\sqrt[3]{1-z}}{2 \sqrt[3]{c z}} .
\end{gathered}
$$

Now, $G_{k}(z)$ can be evaluated by residues theorem. Suppose first that $k \geq 0$ (then $k+N-1 \geq 0$ ) so that 0 is not a pole in the integral defining $G_{k}(z)$ :

$$
\begin{aligned}
G_{k}(z) & =\sum_{j=1}^{N} \operatorname{Res}\left(\frac{\zeta^{k+N-1}}{P(z, \zeta)}, \zeta=u_{j}(z)\right) \\
& =\sum_{j=1}^{N} \frac{u_{j}(z)^{k+N-1}}{(\partial P / \partial \zeta)\left(z, u_{j}(z)\right)} \\
& =\frac{1}{N(1-z)} \sum_{j=1}^{N} \frac{1-u_{j}(z)}{1+u_{j}(z)} u_{j}(z)^{k}
\end{aligned}
$$

The foregoing representation of $G_{k}(z)$ is valid a priori for any $z \in\left(0,1 / M_{\infty}\right)$. Actually, in view of the expressions of $w(z)$ and $u_{j}(z)$, we can see that (93) defines an analytical function in the interval $(0,1)$. Since $G_{k}(z)$ is a power series, by analytical continuation, equality (93) holds true for any $z \in$ $(0,1)$. Moreover, by symmetry, we have that $G_{k}(z)=G_{-k}(z)$ for $k \leq 0$. We display this result in the theorem below.

Theorem 11. For any $k \in \mathbb{Z}$, the generating function of the $\mathbb{P}\left\{S_{n}=k\right\}, n \in \mathbb{N}$, is given, for any $z \in(0,1)$, by

$$
G_{k}(z)=\frac{1}{N(1-z)} \sum_{j=1}^{N} \frac{1-u_{j}(z)}{1+u_{j}(z)} u_{j}(z)^{|k|}
$$

Remark 12. Another proof of Theorem 11 consists in expanding the rational fraction $\zeta \mapsto G(z, \zeta)$ into partial fractions. We find it interesting to outline the main steps of this method. We can write that

$$
G(z, \zeta)=\sum_{j=1}^{N} \frac{u_{j}(z)}{\zeta-u_{j}(z)}+\sum_{j=1}^{N} \frac{v_{j}(z)}{\zeta-v_{j}(z)}
$$

with

$$
\begin{gathered}
u_{j}(z)=\frac{u_{j}(z)}{N(1-z)} \frac{1-u_{j}(z)}{1+u_{j}(z)}, \\
v_{j}(z)=\frac{v_{j}(z)}{N(1-z)} \frac{1-v_{j}(z)}{1+v_{j}(z)}=-\frac{1 / u_{j}(z)}{N(1-z)} \frac{1-u_{j}(z)}{1+u_{j}(z)} .
\end{gathered}
$$

We next expand the partial fractions $1 /\left(\zeta-u_{j}(z)\right)$ and $1 /(\zeta-$ $\left.v_{j}(z)\right)$ into power series as follows. We have checked that $\left|u_{j}(z)\right|<1<\left|v_{j}(z)\right|$ for any $j \in\{1, \ldots, N\}$. Now, if $\left|u_{j}(z)\right|<$ $|\zeta|<\left|v_{j}(z)\right|$ for any $j \in\{1, \ldots, N\}$,

$$
\begin{gathered}
\frac{1}{\zeta-u_{j}(z)}=\sum_{k=0}^{\infty} \frac{u_{j}(z)^{k}}{\zeta^{k+1}}=\sum_{k=-\infty}^{-1} \frac{\zeta^{k}}{u_{j}(z)^{k+1}}, \\
\frac{1}{\zeta-v_{j}(z)}=-\sum_{k=0}^{\infty} \frac{\zeta^{k}}{v_{j}(z)^{k+1}}
\end{gathered}
$$

from which (94) can be easily extracted. 
2.3. Limiting Pseudoprocess. In this section, by pseudoprocess it is meant a continuous-time process driven by a signed measure. Actually, this object is not properly defined on all continuous times but only on dyadic times $k / 2^{j}, j, k \in \mathbb{N}$. A proper definition consists in seeing it as the limit of a step process associated with the observations of the pseudoprocess on the dyadic times. We refer the reader to $[10,18]$ for precise details which are cumbersome to reproduce here.

Below, we give an ad hoc definition for the convergence of a family of pseudoprocesses $\left(\left(X_{t}^{\varepsilon}\right)_{t \geq 0}\right)_{\varepsilon>0}$ towards a pseudoprocess $\left(X_{t}\right)_{t \geq 0}$.

Definition 13. Let $\left(\left(X_{t}^{\varepsilon}\right)_{t \geq 0}\right)_{\varepsilon>0}$ be a family of pseudoprocesses and $\left(X_{t}\right)_{t \geq 0}$ a pseudoprocess. We say that

$$
\left(X_{t}^{\varepsilon}\right)_{t \geq 0} \underset{\varepsilon \rightarrow 0^{+}}{\longrightarrow}\left(X_{t}\right)_{t \geq 0}
$$

if and only if

$$
\begin{gathered}
\forall n \in \mathbb{N}^{*}, \quad \forall t_{1}, \ldots, t_{n} \geq 0, \quad \forall \mu_{1}, \ldots, \mu_{n} \in \mathbb{R}, \\
\mathbb{E}\left(\mathrm{e}^{i \sum_{k=1}^{n} \mu_{k} X_{t_{k}}^{\varepsilon}}\right) \underset{\varepsilon \rightarrow 0^{+}}{\longrightarrow} \mathbb{E}\left(\mathrm{e}^{i \sum_{k=1}^{n} \mu_{k} X_{t_{k}}}\right) .
\end{gathered}
$$

This is the weak convergence of the finite-dimensional projections of the family of pseudoprocesses.

In this part, we choose for the family $\left(\left(X_{t}^{\varepsilon}\right)_{t \geq 0}\right)_{\varepsilon>0}$ the continuous-time pseudoprocesses defined, for any $\varepsilon>0$, by

$$
X_{t}^{\varepsilon}=\varepsilon S_{\left\lfloor t / \varepsilon^{2 N}\right\rfloor}, \quad t \geq 0
$$

where $\lfloor\cdot\rfloor$ stands for the usual floor function. The quantity $X_{t}^{\varepsilon}$ takes its values on the discrete set $\varepsilon \mathbb{Z}$. Roughly speaking, we normalize the pseudorandom walk on the time $\times$ space grid $\varepsilon^{2 N} \mathbb{N} \times \varepsilon \mathbb{Z}$. Let $\left(X_{t}\right)_{t \geq 0}$ be the pseudo-Brownian motion. It is characterized by the following property: for any $n \in \mathbb{N}^{*}$, any $t_{1}, \ldots, t_{n} \geq 0$ such that $t_{1}<\cdots<t_{n}$ and any $\mu_{1}, \ldots, \mu_{n} \in \mathbb{R}$,

$$
\mathbb{E}\left(\mathrm{e}^{i \sum_{k=1}^{n} \mu_{k} X_{t_{k}}}\right)=\mathrm{e}^{-c \sum_{k=1}^{n}\left(\mu_{1}+\cdots+\mu_{k}\right)^{2 N}\left(t_{k}-t_{k-1}\right)} .
$$

We refer to $[10,18]$ for a proper definition of pseudoBrownian motion, and to references therein for interesting properties of this pseudoprocess.

Theorem 14. Suppose that $c \leq 1 / 2^{2 N-1}$. The following convergence holds:

$$
\left(X_{t}^{\varepsilon}\right)_{t \geq 0} \underset{\varepsilon \rightarrow 0^{+}}{\longrightarrow}\left(X_{t}\right)_{t \geq 0}
$$

Proof. (i) We begin by computing the Laplace-Fourier transform of $X_{t}^{\varepsilon}$. By definition of $X_{t}^{\varepsilon}$, we have that $\mathbb{E}\left(\mathrm{e}^{i \mu X_{t}^{\varepsilon}}\right)=$ $\mathbb{E}\left(\mathrm{e}^{i \mu \varepsilon S_{\left\lfloor t / \varepsilon^{2 N}\right\rfloor}}\right)$ and then

$$
\begin{aligned}
\int_{0}^{+\infty} & \mathrm{e}^{-\lambda t} \mathbb{E}\left(\mathrm{e}^{i \mu X_{t}^{\varepsilon}}\right) \mathrm{d} t \\
& =\sum_{n=0}^{\infty}\left(\int_{n \varepsilon^{2 N}}^{(n+1) \varepsilon^{2 N}} \mathrm{e}^{-\lambda t} \mathrm{~d} t\right) \mathbb{E}\left(\mathrm{e}^{i \mu \varepsilon S_{n}}\right) \\
& =\frac{1-\mathrm{e}^{-\lambda \varepsilon^{2 N}}}{\lambda} \sum_{n=0}^{\infty}\left(\mathrm{e}^{-\lambda \varepsilon^{2 N}}\right)^{n} \mathbb{E}\left(\mathrm{e}^{i \mu \varepsilon S_{n}}\right) \\
& =\frac{1-\mathrm{e}^{-\lambda \varepsilon^{2 N}}}{\lambda} \sum_{n \in \mathbb{N}, k \in \mathbb{Z}}\left(\mathrm{e}^{-\lambda \varepsilon^{2 N}}\right)^{n}\left(\mathrm{e}^{i \mu \varepsilon}\right)^{k} \mathbb{P}\left\{S_{n}=k\right\} \\
& =\frac{1-\mathrm{e}^{-\lambda \varepsilon^{2 N}}}{\lambda} G\left(\mathrm{e}^{-\lambda \varepsilon^{2 N}}, \mathrm{e}^{i \mu \varepsilon}\right) .
\end{aligned}
$$

By (71), we have that

$$
G\left(\mathrm{e}^{-\lambda \varepsilon^{2 N}}, \mathrm{e}^{i \mu \varepsilon}\right)=\frac{1}{1-\mathrm{e}^{-\lambda \varepsilon^{2 N}}+c 4^{N} \mathrm{e}^{-\lambda \varepsilon^{2 N}} \sin ^{2 N}(\mu \varepsilon / 2)}
$$

Actually, equality (104) is valid for $\lambda$ such that $\mathrm{e}^{-\lambda \varepsilon^{2 N}}<1 / M_{\infty}$; that is, $\lambda>\left(\ln M_{\infty}\right) / \varepsilon^{2 N}$. Since $c$ is assumed not to be greater than $1 / 2^{2 N-1}$, by (42), we have that $M_{\infty}=1$ and (104) is valid for any $\lambda>0$.

Now, by using the elementary asymptotics $\sin (\mu \varepsilon / 2) \underset{\varepsilon \rightarrow 0^{+}}{=}$ $\mu \varepsilon / 2+o(\varepsilon)$ and $\mathrm{e}^{-\lambda \varepsilon^{2 N}} \underset{\varepsilon \rightarrow 0^{+}}{=} 1-\lambda \varepsilon^{2 N}+o(\varepsilon)$, we obtain that

$$
G\left(\mathrm{e}^{-\lambda \varepsilon^{2 N}}, \mathrm{e}^{i \mu \varepsilon}\right) \underset{\varepsilon \rightarrow 0^{+}}{\sim} \frac{1}{\lambda+c \mu^{2 N}} \frac{1}{\varepsilon^{2 N}} .
$$

As a result, for any $\lambda>0$,

$$
\lim _{\varepsilon \rightarrow 0^{+}} \int_{0}^{+\infty} \mathrm{e}^{-\lambda t} \mathbb{E}\left(\mathrm{e}^{i \mu X_{t}^{\varepsilon}}\right) \mathrm{d} t=\frac{1}{\lambda+c \mu^{2 N}}=\int_{0}^{+\infty} \mathrm{e}^{-\left(\lambda+c \mu^{2 N}\right) t} \mathrm{~d} t
$$

from which and (101) we deduce that

$$
\lim _{\varepsilon \rightarrow 0^{+}} \mathbb{E}\left(\mathrm{e}^{i \mu X_{t}^{\varepsilon}}\right)=\mathrm{e}^{-c \mu^{2 N} t}=\mathbb{E}\left(\mathrm{e}^{i \mu X_{t}}\right) .
$$

Notice that the Laplace-Fourier of $X_{t}$ takes the simple form

$$
\int_{0}^{+\infty} \mathrm{e}^{-\lambda t} \mathbb{E}\left(\mathrm{e}^{i \mu X_{t}}\right) \mathrm{d} t=\frac{1}{\lambda+c \mu^{2 N}}
$$


(ii) In the same way, we compute the Laplace-Fourier transform of $X_{t+\varepsilon^{2 n}}^{\varepsilon}$ which will be used further. We have $\mathbb{E}\left(\mathrm{e}^{i \mu X_{t+\varepsilon^{2 n}}^{\varepsilon n}}\right)=\mathbb{E}\left(\mathrm{e}^{i \mu \varepsilon S_{\left\lfloor t / \varepsilon^{2 N}\right\rfloor+1}}\right)$. Then

$$
\begin{aligned}
& \int_{0}^{+\infty} \mathrm{e}^{-\lambda t} \mathbb{E}\left(\mathrm{e}^{i \mu X_{t+\varepsilon^{2 n}}}\right) \mathrm{d} t \\
& =\sum_{n=0}^{\infty}\left(\int_{n \varepsilon^{2 N}}^{(n+1) \varepsilon^{2 N}} \mathrm{e}^{-\lambda t} \mathrm{~d} t\right) \mathbb{E}\left(\mathrm{e}^{\mathrm{i} \mu \varepsilon S_{\mathrm{n}+1}}\right) \\
& =\frac{1-\mathrm{e}^{-\lambda \varepsilon^{2 N}}}{\lambda} \sum_{n=0}^{\infty}\left(\mathrm{e}^{-\lambda \varepsilon^{2 N}}\right)^{n} \mathbb{E}\left(\mathrm{e}^{i \mu \varepsilon S_{n+1}}\right) \\
& =\frac{1-\mathrm{e}^{-\lambda \varepsilon^{2 N}}}{\lambda} \mathrm{e}^{\lambda \varepsilon^{2 N}} \sum_{n \in \mathbb{N}^{*}, k \in \mathbb{Z}}\left(\mathrm{e}^{-\lambda \varepsilon^{2 N}}\right)^{n}\left(\mathrm{e}^{i \mu \varepsilon}\right)^{k} \mathbb{P}\left\{S_{n}=k\right\} \\
& =\frac{\mathrm{e}^{\lambda \varepsilon^{2 N}}-1}{\lambda}\left[G\left(\mathrm{e}^{-\lambda \varepsilon^{2 N}}, \mathrm{e}^{i \mu \varepsilon}\right)-1\right] .
\end{aligned}
$$

As for (107), we immediately extract the following limit:

$$
\lim _{\varepsilon \rightarrow 0^{+}} \mathbb{E}\left(\mathrm{e}^{i \mu X_{t+\varepsilon^{\varepsilon}}^{\varepsilon N}}\right)=\mathbb{E}\left(\mathrm{e}^{i \mu X_{t}}\right) .
$$

(iii) We now compute the joint Fourier transform of $\left(X_{t_{1}}^{\varepsilon}, X_{t_{2}}^{\varepsilon}\right)$ for two times $t_{1}, t_{2}$ such that $t_{1}<t_{2}$. Using the elementary fact that $\lfloor x\rfloor-\lfloor y\rfloor \in\{\lfloor x-y\rfloor,\lfloor x-y\rfloor+1\}$, we observe that

$$
\begin{aligned}
X_{t_{2}}^{\varepsilon}-X_{t_{1}}^{\varepsilon} & \stackrel{d}{=} \varepsilon S_{\left\lfloor t_{2} / \varepsilon^{2 N}\right\rfloor-\left\lfloor t_{1} / \varepsilon^{2 N}\right\rfloor} \in\left\{\varepsilon S_{\left\lfloor\left(t_{2}-t_{1}\right) / \varepsilon^{2 N}\right\rfloor}, \varepsilon S_{\left\lfloor\left(t_{2}-t_{1}\right) / \varepsilon^{2 N}\right\rfloor+1}\right\} \\
& =\left\{X_{t_{2}-t_{1}}^{\varepsilon}, X_{t_{2}-t_{1}+\varepsilon^{2 N}}^{\varepsilon}\right\} .
\end{aligned}
$$

Then, we get, for $\mu_{1}, \mu_{2} \in \mathbb{R}$, that

$$
\begin{aligned}
\mathbb{E}\left(\mathrm{e}^{i\left(\mu_{1} X_{t_{1}}^{\varepsilon}+\mu_{2} X_{t_{2}}^{\varepsilon}\right)}\right) \in\{ & \mathbb{E}\left(\mathrm{e}^{i\left(\mu_{1}+\mu_{2}\right) X_{t_{1}}^{\varepsilon}}\right) \mathbb{E}\left(\mathrm{e}^{i \mu_{2} X_{t_{2}-t_{1}}^{\varepsilon}}\right), \\
& \left.\mathbb{E}\left(\mathrm{e}^{i\left(\mu_{1}+\mu_{2}\right) X_{t_{1}}^{\varepsilon}}\right) \mathbb{E}\left(\mathrm{e}^{i \mu_{2} X_{t_{2}-t_{1}+\varepsilon^{2 N}}^{\varepsilon}}\right)\right\} .
\end{aligned}
$$

By (107) and (110), we obtain the following limit:

$$
\lim _{\varepsilon \rightarrow 0^{+}} \mathbb{E}\left(\mathrm{e}^{i \mu_{2} X_{t_{2}-t_{1}}^{\varepsilon}}\right)=\lim _{\varepsilon \rightarrow 0^{+}} \mathbb{E}\left(\mathrm{e}^{i \mu_{2} X_{t_{2}-t_{1}+\varepsilon^{2 N}}^{2 N}}\right)=\mathbb{E}\left(\mathrm{e}^{i \mu_{2} X_{t_{2}-t_{1}}}\right)
$$

which yields that

$$
\begin{aligned}
\lim _{\varepsilon \rightarrow 0^{+}} & \mathbb{E}\left(\mathrm{e}^{i\left(\mu_{1} X_{t_{1}}^{\varepsilon}+\mu_{2} X_{t_{2}}^{\varepsilon}\right)}\right) \\
& =\lim _{\varepsilon \rightarrow 0^{+}} \mathbb{E}\left(e^{i\left(\mu_{1}+\mu_{2}\right) X_{t_{1}}^{\varepsilon}}\right) \times \lim _{\varepsilon \rightarrow 0^{+}} \mathbb{E}\left(e^{i \mu_{2} X_{t_{2}-t_{1}}^{\varepsilon}}\right) \\
& =\mathbb{E}\left(\mathrm{e}^{i\left(\mu_{1}+\mu_{2}\right) X_{t_{1}}}\right) \times \mathbb{E}\left(\mathrm{e}^{i \mu_{2} X_{t_{2}-t_{1}}}\right) \\
& =\mathbb{E}\left(\mathrm{e}^{i\left(\mu_{1} X_{t_{1}}+\mu_{2} X_{t_{2}}\right)}\right) .
\end{aligned}
$$

(iv) Finally, we can easily extend the foregoing limiting result by recurrence as follows: for $n \in \mathbb{N}^{*}, \mu_{1}, \ldots, \mu_{n} \in \mathbb{R}$ and for any times $t_{1}, \ldots, t_{n}$ such that $t_{1}<\cdots<t_{n}$,

$$
\lim _{\varepsilon \rightarrow 0^{+}} \mathbb{E}\left(\mathrm{e}^{i\left(\mu_{1} X_{t_{1}}^{\varepsilon}+\cdots+\mu_{n} X_{t_{n}}^{\varepsilon}\right)}\right)=\mathbb{E}\left(\mathrm{e}^{i\left(\mu_{1} X_{t_{1}}+\cdots+\mu_{n} X_{t_{n}}\right)}\right) .
$$

The proof of Theorem 14 is complete.

We find it interesting to compute in a similar way the $\lambda$ potential of the pseudoprocess $\left(X_{t}\right)_{t \geq 0}$. By definition of $X_{t}^{\varepsilon}$, we have, for any $\alpha, \beta \in \mathbb{R}$ such that $\alpha<\beta, \mathbb{P}\left\{X_{t}^{\varepsilon} \in[\alpha, \beta)\right\}=$ $\mathbb{P}\left\{S_{\left\lfloor t / \varepsilon^{2 N}\right\rfloor} \in[\alpha / \varepsilon, \beta / \varepsilon)\right\}$. Thus,

$$
\begin{aligned}
\int_{0}^{+\infty} & \mathrm{e}^{-\lambda t} \mathbb{P}\left\{X_{t}^{\varepsilon} \in[\alpha, \beta)\right\} \mathrm{d} t \\
& =\sum_{n=0}^{\infty}\left(\int_{n \varepsilon^{2 N}}^{(n+1) \varepsilon^{2 N}} \mathrm{e}^{-\lambda t} \mathrm{~d} t\right) \mathbb{P}\left\{S_{n} \in\left[\frac{\alpha}{\varepsilon}, \frac{\beta}{\varepsilon}\right)\right\} \\
& =\frac{1-\mathrm{e}^{-\lambda \varepsilon^{2 N}}}{\lambda} \sum_{n=0}^{\infty}\left(\mathrm{e}^{-\lambda \varepsilon^{2 N}}\right)^{n}\left[\sum_{\substack{k \in \mathbb{Z}: \\
\alpha / \varepsilon \leq k<\beta / \varepsilon}} \mathbb{P}\left\{S_{n}=k\right\}\right] \\
= & \frac{1-\mathrm{e}^{-\lambda \varepsilon^{2 N}}}{\lambda} \sum_{\substack{k \in \mathbb{Z}: \\
\alpha / \varepsilon \leq k<\beta / \varepsilon}}\left[\sum_{n=0}^{\infty} \mathbb{P}\left\{S_{n}=k\right\}\left(\mathrm{e}^{-\lambda \varepsilon^{2 N}}\right)^{n}\right] \\
= & \frac{1-\mathrm{e}^{-\lambda \varepsilon^{2 N}}}{\lambda} \sum_{\substack{k \in \mathbb{Z}: \\
\alpha / \varepsilon \leq k<\beta / \varepsilon}} G_{k}\left(\mathrm{e}^{-\lambda \varepsilon^{2 N}}\right) .
\end{aligned}
$$

Interchanging the two sums in the above computations is justified by the fact that the series $\sum_{n=0}^{\infty} \mathbb{P}\left\{S_{n}=k\right\}\left(\mathrm{e}^{-\lambda \varepsilon^{2 N}}\right)^{n}$ is absolutely convergent because of the condition $c \leq 1 / 2^{2 N-1}$. Indeed, by (53), for any $\lambda>0,\left|\mathbb{P}\left\{S_{n}=k\right\}\right|<M_{\infty}^{n}=1$.

Put $u_{j}(\lambda, \varepsilon)=u_{j}\left(\mathrm{e}^{-\lambda \varepsilon^{2 N}}\right)$. This yields that

$$
\begin{aligned}
\int_{0}^{+\infty} & \mathrm{e}^{-\lambda t} \mathbb{P}\left\{X_{t}^{\varepsilon} \in[\alpha, \beta)\right\} \mathrm{d} t \\
& =\frac{1}{N \lambda} \sum_{j=1}^{N} \frac{1-u_{j}(\lambda, \varepsilon)}{1+u_{j}(\lambda, \varepsilon)} \sum_{\alpha / \varepsilon \leq k<\beta / \varepsilon} u_{j}(\lambda, \varepsilon)^{|k|} .
\end{aligned}
$$

Suppose, for example, that $0 \leq \alpha<\beta$. Then,

$$
\begin{aligned}
\sum_{\alpha / \varepsilon \leq k<\beta / \varepsilon} u_{j}(\lambda, \varepsilon)^{|k|} & =\sum_{k=\lceil\alpha / \varepsilon\rceil}^{\lceil\beta / \varepsilon\rceil-1} u_{j}(\lambda, \varepsilon)^{k} \\
& =\frac{u_{j}(\lambda, \varepsilon)^{\lceil\alpha / \varepsilon\rceil}-u_{j}(\lambda, \varepsilon)^{\lceil\beta / \varepsilon\rceil}}{1-u_{j}(\lambda, \varepsilon)},
\end{aligned}
$$

where $\lceil\cdot\rceil$ stands for the usual ceiling function. By using (85), we deduce that

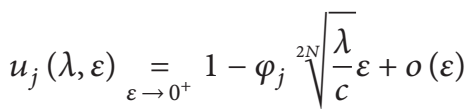


which implies that

$$
\lim _{\varepsilon \rightarrow 0^{+}} u_{j}(\lambda, \varepsilon)^{\lceil\alpha / \varepsilon\rceil}=\mathrm{e}^{-\varphi_{j} \sqrt[2 N]{\lambda / c \alpha}} .
$$

Therefore,

$$
\begin{aligned}
& \lim _{\varepsilon \rightarrow 0^{+}} \int_{0}^{+\infty} \mathrm{e}^{-\lambda t} \mathbb{P}\left\{X_{t}^{\varepsilon} \in[\alpha, \beta)\right\} \mathrm{d} t \\
& \quad=\frac{1}{2 N \lambda} \sum_{j=1}^{N}\left(\mathrm{e}^{-\varphi_{j} \sqrt[2 N]{\lambda / c \alpha}}-\mathrm{e}^{-\varphi_{j} \sqrt[2 N]{\lambda / c} \beta}\right) \\
& =\frac{1}{2 N \lambda} \sum_{j=1}^{N}\left(\varphi_{j} \sqrt[2 N]{\frac{\lambda}{c}} \int_{\alpha}^{\beta} \mathrm{e}^{-\varphi_{j} \sqrt{2 N} \sqrt{\lambda / c} x} \mathrm{~d} x\right) .
\end{aligned}
$$

The case $\alpha<\beta \leq 0$ is similar to treat. We have obtained the following result.

Proposition 15. The $\lambda$-potential of the pseudoprocess $\left(X_{t}\right)_{t \geq 0}$ is given by

$$
\begin{aligned}
& \int_{0}^{+\infty} \mathrm{e}^{-\lambda t}\left(\frac{\mathbb{P}\left\{X_{t} \in \mathrm{d} x\right\}}{\mathrm{d} x}\right) \mathrm{d} t \\
& \quad= \begin{cases}\frac{1}{2 N^{2 N} \sqrt{c} \lambda^{1-1 /(2 N)}} \sum_{j=1}^{N} \varphi_{j} \mathrm{e}^{-\varphi_{j} \sqrt[2 N]{\lambda / c} x} & \text { if } x \geq 0, \\
-\frac{1}{2 N \sqrt[2 N]{c} \lambda^{1-1 /(2 N)}} \sum_{j=1}^{N} \varphi_{j} \mathrm{e}^{\varphi_{j} \sqrt[2 N]{\lambda / c} x} & \text { if } x \leq 0 .\end{cases}
\end{aligned}
$$

\section{Part II-First Overshooting Time of a Single Threshold}

3.1. On the Pseudodistribution of $\left(\sigma_{b}^{+}, S_{b}^{+}\right)$. Let $b \in \mathbb{N}^{*}$. In this section, we explicitly compute the generating function of $\left(\sigma_{b}^{+}, S_{b}^{+}\right)$. Set, for $\ell \in\{b, b+1, \ldots, b+N-1\}$,

$$
H_{b, \ell}^{+}(z)=\mathbb{E}\left(z^{\sigma_{b}^{+}} \mathbb{1}_{\left\{S_{b}^{+}=\ell, \sigma_{b}^{+}<+\infty\right\}}\right)=\sum_{k \in \mathbb{N}} \mathbb{P}\left\{\sigma_{b}^{+}=k, S_{b}^{+}=\ell\right\} z^{k}
$$

We are able to provide an explicit expression of $\mathrm{H}_{b, \ell}^{+}(z)$. Before tackling this problem, we need an a priori estimate for $\mathbb{P}\left\{\sigma_{b}^{+}=\right.$ $\left.k, S_{b}^{+}=\ell\right\}$. By (62), we immediately derive that $\mid \mathbb{P}\left\{\sigma_{b}^{+}=k\right.$, $\left.S_{b}^{+}=\ell\right\}|=| \mathbb{P}\left\{S_{1}<b, \ldots, S_{k-1}<b, S_{k}=\ell\right\} \mid \leq M_{1}^{k}$. Hence, the power series defining $H_{b, \ell}^{+}(z)$ absolutely converges for $|z|<$ $1 / M_{1}$.

\subsubsection{Joint Pseudodistribution of $\left(\sigma_{b}^{+}, S_{b}^{+}\right)$}

Theorem 16. The pseudodistribution of $\left(\sigma_{b}^{+}, S_{b}^{+}\right)$is characterized by the identity, valid for any $z \in(0,1)$ and any $\ell \in$ $\{b, b+1, \ldots, b+N-1\}$,

$$
\mathbb{E}\left(z^{\sigma_{b}^{+}} \mathbb{1}_{\left\{S_{b}^{+}=\ell, \sigma_{b}^{+}<+\infty\right\}}\right)=(-1)^{\ell-b} \sum_{k=1}^{N} \frac{s_{k, \ell-b}^{+}(z)}{p_{k}^{+}(z)} u_{k}(z)^{b+N-1},
$$

where $s_{k, 0}^{+}(z)=1$ and for $k \in\{1, \ldots, N\}, \ell \in\{1, \ldots, N-1\}$,

$$
\begin{gathered}
s_{k, \ell}^{+}(z)=\sum_{\substack{1 \leq i_{1}<\cdots<i_{\ell} \leq N \\
i_{1}, \ldots, i_{\ell} \neq k}} u_{i_{1}}(z) \cdots u_{i_{\ell}}(z), \\
p_{k}^{+}(z)=\prod_{\substack{1 \leq j \leq N \\
j \neq k}}\left[u_{k}(z)-u_{j}(z)\right] .
\end{gathered}
$$

Proof. Pick an integer $k \geq b$. If $S_{n}=k$, then an overshoot of the threshold $b$ occurs before time $n: \sigma_{b}^{+} \leq n$. This remark and the independence of the increments of the pseudorandom walk entail that

$$
\begin{aligned}
\mathbb{P}\left\{S_{n}=k\right\} & =\mathbb{P}\left\{S_{n}=k, \sigma_{b}^{+} \leq n\right\} \\
& =\sum_{j=0}^{n} \sum_{\ell=b}^{b+N-1} \mathbb{P}\left\{S_{n}=k, \sigma_{b}^{+}=j, S_{b}^{+}=\ell\right\} \\
& =\sum_{j=0}^{n} \sum_{\ell=b}^{b+N-1} \mathbb{P}\left\{\sigma_{b}^{+}=j, S_{b}^{+}=\ell\right\} \mathbb{P}\left\{S_{n-j}=k-\ell\right\} .
\end{aligned}
$$

Since the series defining $G_{k}(z)$ and $H_{b, e}^{+}(z)$ absolutely converge, respectively, for $z \in(0,1)$ and $|z|<1 / M_{1}$, and since $M_{1} \geq 1$, we can apply the generating function to the convolution equality (126). We get, for $z \in\left(0,1 / M_{1}\right)$, that

$$
G_{k}(z)=\sum_{\ell=b}^{b+N-1} G_{k-\ell}(z) H_{b, \ell}^{+}(z) .
$$

Using expression (94) of $G_{k}$, namely, $G_{k}(z)=$ $\sum_{j=1}^{N} \alpha_{j}(z) u_{j}(z)^{k}$ for $k \geq 0$, where $\alpha_{j}(z)=1 /[N(1-z)] \times$ $\left[1-u_{j}(z)\right] /\left[1+u_{j}(z)\right]$, we obtain that

$$
\sum_{j=1}^{N} \alpha_{j}(z) u_{j}(z)^{k}\left(\sum_{\ell=b}^{b+N-1} \frac{H_{b, \ell}^{+}(z)}{u_{j}(z)^{\ell}}-1\right)=0, \quad k \geq b+N-1 .
$$

Recalling that $v_{j}(z)=1 / u_{j}(z)$ and setting $\widetilde{\alpha}_{j}(z)=$ $\alpha_{j}(z)\left(\sum_{\ell=b}^{b+N-1} H_{b, \ell}^{+}(z) v_{j}(z)^{\ell}-1\right)$, system (128) reads $\sum_{j=1}^{N} \widetilde{\alpha}_{j}(z) u_{j}(z)^{k}=0, k \geq b+N-1$. When limiting the range of $k$ to the set $\{b+N, b+N+1, \ldots, b+2 N-1\}$, this becomes a homogeneous Vandermonde system whose solution is trivial: $\widetilde{\alpha}_{j}(z)=0,1 \leq j \leq N$. Thus, we get the following Vandermonde system:

$$
\sum_{\ell=b}^{b+N-1} H_{b, \ell}^{+}(z) v_{j}(z)^{\ell}=1, \quad 1 \leq j \leq N .
$$

System (129) can be explicitly solved. In order to simplify the settings, we will omit the variable $z$ in the sequel of the proof. It is convenient to rewrite (129) as

$$
\sum_{\ell=b}^{b+N-1} H_{b, \ell}^{+} e_{j}^{\ell-b}=u_{j}^{b}, \quad 1 \leq j \leq N .
$$


Cramer's formulae yield

$$
H_{b, \ell}^{+}=\frac{V_{\ell}\left(v_{1}, \ldots, v_{N}\right)}{V\left(v_{1}, \ldots, v_{N}\right)}, \quad b \leq \ell \leq b+N-1 \text {, }
$$

where

$$
V\left(v_{1}, \ldots, v_{N}\right)=\left|\begin{array}{cccc}
1 & v_{1} & \cdots & v_{1}^{N-1} \\
1 & v_{2} & \cdots & v_{2}^{N-1} \\
\vdots & \vdots & & \vdots \\
1 & v_{N} & \cdots & v_{N}^{N-1}
\end{array}\right|=\prod_{1 \leq i<j \leq N}\left(v_{j}-v_{i}\right)
$$

and, for any $\ell \in\{b, \ldots, b+N-1\}$,

$$
V_{\ell}\left(v_{1}, \ldots, v_{N}\right)=\left|\begin{array}{cccccccc}
1 & v_{1} & \cdots & v_{1}^{\ell-b-1} & u_{1}^{b} & v_{1}^{\ell-b+1} & \cdots & v_{1}^{N-1} \\
1 & v_{2} & \cdots & v_{2}^{\ell-b-1} & u_{2}^{b} & v_{2}^{\ell-b+1} & \cdots & v_{2}^{N-1} \\
\vdots & \vdots & & \vdots & \vdots & \vdots & & \vdots \\
1 & v_{N} & \cdots & v_{N}^{\ell-b-1} & u_{N}^{b} & v_{N}^{\ell-b+1} & \cdots & v_{N}^{N-1}
\end{array}\right| .
$$

This last determinant can be expanded as $\sum_{k=1}^{N} u_{k}^{b} V_{k \ell}\left(v_{1}, \ldots\right.$, $\left.v_{k-1}, v_{k+1}, \ldots, v_{N}\right)$ with, for $k \in\{1, \ldots, N\}$,

$$
\begin{aligned}
V_{k \ell}\left(v_{1}, \ldots, v_{k-1}, v_{k+1}, \ldots,\right. & \left.v_{N}\right) \\
= & \left|\begin{array}{cccccccc}
1 & v_{1} & \cdots & v_{1}^{\ell-b-1} & 0 & v_{1}^{\ell-b+1} & \cdots & v_{1}^{N-1} \\
\vdots & \vdots & & \vdots & \vdots & \vdots & & \vdots \\
1 & v_{k-1} & \cdots & v_{k-1}^{\ell-b-1} & 0 & v_{k-1}^{\ell-b+1} & \cdots & v_{k-1}^{N-1} \\
1 & v_{k} & \cdots & v_{k}^{\ell-b-1} & 1 & v_{k}^{\ell-b+1} & \cdots & v_{k}^{N-1} \\
1 & v_{k+1} & \cdots & v_{k+1}^{\ell-b-1} & 0 & v_{k+1}^{\ell-b+1} & \cdots & v_{k+1}^{N-1} \\
\vdots & \vdots & & \vdots & \vdots & \vdots & & \vdots \\
1 & v_{N} & \cdots & v_{N}^{\ell-b-1} & 0 & v_{N}^{\ell-b+1} & \cdots & v_{N}^{N-1}
\end{array}\right| .
\end{aligned}
$$

In fact, the quantity $V_{k \ell}\left(v_{1}, \ldots, v_{k-1}, v_{k+1}, \ldots, v_{N}\right)$ is the coefficient of $x^{\ell-b}$ in the polynomial

$$
x \longmapsto\left|\begin{array}{cccc}
1 & v_{1} & \cdots & v_{1}^{N-1} \\
\vdots & \vdots & & \vdots \\
1 & v_{k-1} & \cdots & v_{k-1}^{N-1} \\
1 & x & \cdots & x^{N-1} \\
1 & v_{k+1} & \cdots & v_{k+1}^{N-1} \\
\vdots & \vdots & & \vdots \\
1 & v_{N} & \cdots & v_{N}^{N-1}
\end{array}\right|
$$

which is nothing but $V\left(v_{1}, \ldots, v_{k-1}, x, v_{k+1}, \ldots, v_{N}\right)$, the value of which is

$$
\begin{aligned}
& \prod_{\substack{1 \leq i<j \leq N \\
i, j \neq k}}\left(v_{j}-v_{i}\right) \prod_{1 \leq i \leq k-1}\left(x-v_{i}\right) \prod_{k+1 \leq i \leq N}\left(v_{i}-x\right) \\
& =\frac{\prod_{1 \leq i<j \leq N}\left(v_{j}-v_{i}\right)}{\prod_{\substack{1 \leq i \leq N \\
i \neq k}}\left(v_{k}-v_{i}\right)} \prod_{\substack{1 \leq i \leq N \\
i \neq k}}\left(x-v_{i}\right) \\
& =V\left(v_{1}, \ldots, v_{N}\right) \prod_{\substack{1 \leq i \leq N \\
i \neq k}} \frac{x-v_{i}}{v_{k}-v_{i}} \\
& =(-1)^{N-1} V\left(v_{1}, \ldots, v_{N}\right) \prod_{\substack{1 \leq i \leq N \\
i \neq k}}\left(u_{k} \frac{u_{i} x-1}{u_{k}-u_{i}}\right)
\end{aligned}
$$

$$
=(-1)^{N-1} \frac{u_{k}^{N-1}}{p_{k}^{+}} V\left(v_{1}, \ldots, v_{N}\right) \prod_{\substack{1 \leq i \leq N \\ i \neq k}}\left(u_{i} x-1\right) .
$$

Using the elementary expansion $\prod_{\substack{1 \leq i \leq N \\ i \neq k}}\left(u_{i} x-1\right)=$ $\sum_{\ell=0}^{N-1}(-1)^{N-1-\ell} s_{k, \ell}^{+} x^{\ell}$, we obtain by identification that

$$
\begin{aligned}
V_{k \ell}( & \left.v_{1}, \ldots, v_{k-1}, v_{k+1}, \ldots, v_{N}\right) \\
& =(-1)^{\ell-b} \frac{u_{k}^{N-1}}{p_{k}^{+}} s_{k, \ell-b}^{+} V\left(v_{1}, \ldots, v_{N}\right)
\end{aligned}
$$

Plugging this expression into (131), we then derive for $\mathrm{H}_{b, \ell}^{+}(z)$ representation (124) which is valid at least for $z \in\left(0,1 / M_{1}\right)$. Finally, we observe that (124) defines an analytical function in $(0,1)$ and that $H_{b, \ell}^{+}(z)$ is a power series. Thus, by analytical continuation, (124) holds true for any $z \in(0,1)$.

Example 17. For $N=1$, the settings of Theorem 16 write $s_{1,0}^{+}(z)=p_{1}^{+}(z)=1$. Then, formula (124) reads

$$
\mathbb{E}\left(z^{\sigma_{b}^{+}} \mathbb{1}_{\left\{S_{b}^{+}=b, \sigma_{b}^{+}<+\infty\right\}}\right)=u_{1}(z)^{b}
$$

where $u_{1}(z)$ is given in Example 10. Of course, in this case, the condition $S_{b}^{+}=b$ is redundant since we are dealing with an ordinary random walk with jumps of one unity at most. When $c=1 / 2$, this is the classical symmetric random walk and (124) recovers the most well-known formula in random walk theory:

$$
\mathbb{E}\left(z^{\sigma_{b}^{+}} \mathbb{1}_{\left\{\sigma_{b}^{+}<+\infty\right\}}\right)=\left(\frac{1-\sqrt{1-z^{2}}}{z}\right)^{b} .
$$

For $N=2$, the settings of Theorem 16 write

$$
\begin{gathered}
s_{1,0}^{+}(z)=s_{2,0}^{+}(z)=1, \quad s_{1,1}^{+}(z)=u_{2}(z), \quad s_{2,1}^{+}(z)=u_{1}(z), \\
p_{1}^{+}(z)=u_{1}(z)-u_{2}(z), \quad p_{2}^{+}(z)=u_{2}(z)-u_{1}(z),
\end{gathered}
$$


where $u_{1}(z)$ and $u_{2}(z)$ are given in Example 10 and (124) reads

$$
\begin{gathered}
\mathbb{E}\left(z^{\sigma_{b}^{+}} \mathbb{1}_{\left\{S_{b}^{+}=b, \sigma_{b}^{+}<+\infty\right\}}\right)=\frac{u_{1}(z)^{b+1}-u_{2}(z)^{b+1}}{u_{1}(z)-u_{2}(z)}, \\
\mathbb{E}\left(z^{\sigma_{b}^{+}} \mathbb{1}_{\left\{S_{b}^{+}=b+1, \sigma_{b}^{+}<+\infty\right\}}\right)=\frac{u_{1}(z) u_{2}(z)^{b+1}-u_{2}(z) u_{1}(z)^{b+1}}{u_{1}(z)-u_{2}(z)} .
\end{gathered}
$$

Remark 18. We have the similar expression related to $\sigma_{a}^{-}$ below. The analogous system to (129) writes as

$$
\sum_{\ell=a-N+1}^{a} H_{a, \ell}^{-}(z) u_{j}(z)^{\ell}=1, \quad 1 \leq j \leq N,
$$

where $H_{a, \ell}^{-}(z)=\mathbb{E}\left(z^{\sigma_{a}^{-}} \mathbb{1}_{\left\{S_{a}^{-}=\ell, \sigma_{a}^{-}<+\infty\right\}}\right)$. The solution is given by

$$
\mathbb{E}\left(z^{\sigma_{a}^{-}} \mathbb{1}_{\left\{S_{a}^{-}=\ell, \sigma_{a}^{-}<+\infty\right\}}\right)=(-1)^{\ell-a} \sum_{k=1}^{N} \frac{s_{k, \ell-a}^{-}(z)}{p_{k}^{-}(z)} u_{k}(z)^{a+N-1},
$$

where $s_{k, 0}^{-}(z)=1$ and, for $k \in\{1, \ldots, N\}, \ell \in\{1, \ldots, N-1\}$,

$$
\begin{gathered}
s_{k, \ell}^{-}(z)=\sum_{\substack{1 \leq i_{1}<\cdots<i_{\ell} \leq N \\
i_{1}, \ldots, i_{\ell} \neq k}} v_{i_{1}}(z) \cdots v_{i_{\ell}}(z), \\
p_{k}^{-}(z)=\prod_{\substack{1 \leq j \leq N \\
j \neq k}}\left[v_{k}(z)-v_{j}(z)\right] .
\end{gathered}
$$

The double generating function of $\left(\sigma_{b}^{+}, S_{b}^{+}\right)$defined by

$$
\mathbb{E}\left(z^{\sigma_{b}^{+}} \zeta^{S_{b}^{+}} \mathbb{1}_{\left\{\sigma_{b}^{+}<+\infty\right\}}\right)=\sum_{\ell=b}^{b+N-1} \mathbb{E}\left(z^{\sigma_{b}^{+}} \mathbb{1}_{\left\{S_{b}^{+}=\ell\right\}}\right) \zeta^{\ell}
$$

admits an interesting representation by means of Lagrange interpolating polynomials that we display in the theorem below.

Theorem 19. The double generating function of $\left(\sigma_{b}^{+}, S_{b}^{+}\right)$is given, for any $z \in\left(0,1 / M_{1}\right)$ and $\zeta \in \mathbb{C}$, by

$$
\mathbb{E}\left(z^{\sigma_{b}^{+}} \zeta^{S_{b}^{+}} \mathbb{1}_{\left\{\sigma_{b}^{+}<+\infty\right\}}\right)=\sum_{k=1}^{N} L_{k}(z, \zeta)\left(u_{k}(z) \zeta\right)^{b}
$$

where

$$
L_{k}(z, \zeta)=\prod_{\substack{1 \leq j \leq N \\ j \neq k}} \frac{\zeta-v_{j}(z)}{v_{k}(z)-v_{j}(z)}, \quad k \in\{1, \ldots, N\}
$$

are the Lagrange interpolating polynomials with respect to the variable $\zeta$ such that $L_{k}\left(z, v_{j}(z)\right)=\delta_{j k}$.
Proof. By (131) and by omitting the variable $z$ as previously mentioned, we have that

$$
\begin{aligned}
\mathbb{E} & \left(z^{\sigma_{b}^{+}} \zeta^{S_{b}^{+}} \mathbb{1}_{\left\{\sigma_{b}^{+}<+\infty\right\}}\right) \\
& =\sum_{\ell=b}^{b+N-1} H_{b, \ell}^{+} \zeta^{\ell}=\sum_{\ell=b}^{b+N-1} \frac{V_{\ell}\left(v_{1}, \ldots, v_{N}\right)}{V\left(v_{1}, \ldots, v_{N}\right)} \zeta^{\ell} \\
& =\sum_{\ell=b}^{b+N-1}\left(\sum_{k=1}^{N} u_{k}^{b} \frac{V_{k \ell}\left(v_{1}, \ldots, v_{k-1}, v_{k+1}, \ldots, v_{N}\right)}{V\left(v_{1}, \ldots, v_{N}\right)}\right) \zeta^{\ell} \\
& =\sum_{k=1}^{N}\left(\sum_{\ell=b}^{b+N-1} \frac{V_{k \ell}\left(v_{1}, \ldots, v_{k-1}, v_{k+1}, \ldots, v_{N}\right)}{V\left(v_{1}, \ldots, v_{N}\right)} \zeta^{\ell-b}\right)\left(u_{k} \zeta\right)^{b} \\
& =\sum_{k=1}^{N} \frac{V\left(v_{1}, \ldots, v_{k-1}, \zeta, v_{k+1}, \ldots, v_{N}\right)}{V\left(v_{1}, \ldots, v_{N}\right)}\left(u_{k} \zeta\right)^{b} .
\end{aligned}
$$

It is clear that the quantity $V\left(v_{1}, \ldots, v_{k-1}, \zeta, v_{k+1}, \ldots, v_{N}\right) /$ $V\left(v_{1}, \ldots, v_{N}\right)$, which explicitly writes as

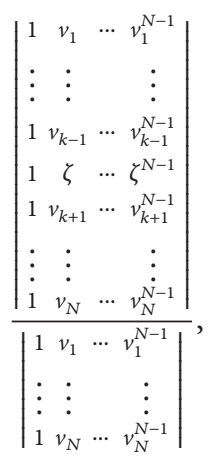

defines a polynomial of the variable $\zeta$ of degree $N-1$ which vanishes at $v_{1}, \ldots, v_{k-1}, v_{k+1}, \ldots, v_{N}$ and equals 1 at $v_{k}$. Hence, by putting back the variable $z$, it coincides with the Lagrange polynomial $L_{k}(z, \zeta)$ and formula (147) immediately ensues.

Example 20. For $N=2$, (147) reads

$$
\begin{aligned}
& \mathbb{E}\left(z^{\sigma_{b}^{+} \zeta^{S_{b}^{+}}} \mathbb{1}_{\left\{\sigma_{b}^{+}<+\infty\right\}}\right) \\
& =\zeta^{b}\left(u_{1}(z)^{b} \frac{\zeta-v_{2}(z)}{v_{1}(z)-v_{2}(z)}+u_{2}(z)^{b} \frac{\zeta-v_{1}(z)}{v_{2}(z)-v_{1}(z)}\right) \\
& =\frac{\zeta^{b}}{u_{1}(z)-u_{2}(z)}\left[\left(u_{1}(z)^{b+1}-u_{2}(z)^{b+1}\right)\right. \\
& \left.\quad+\left(u_{1}(z) u_{2}(z)^{b+1}-u_{2}(z) u_{1}(z)^{b+1}\right) \zeta\right] .
\end{aligned}
$$

This is in good agreement with the formulae of Example 17. We retrieve a result of [21].

3.1.2. Pseudodistribution of $S_{b}^{+}$. In order to derive the pseudodistribution of $S_{b}^{+}$which is characterized by the numbers $H_{b, \ell}^{+}(1), \ell \in\{b, b+1, \ldots, b+N-1\}$, we solve the system obtained by taking the limit in (129) as $z \rightarrow 1^{-}$. 
Lemma 21. The following system holds:

$$
\begin{aligned}
& \sum_{\ell=k+b}^{b+N-1}\left(\begin{array}{c}
\ell-b \\
k
\end{array}\right) H_{b, \ell}^{+}(1) \\
& \quad=(-1)^{k}\left(\begin{array}{c}
b+k-1 \\
b-1
\end{array}\right), \quad 0 \leq k \leq N-1 .
\end{aligned}
$$

Proof. By (85), we have the expansion $v_{j}(z)=1 / u_{j}(z)=1-$ $\varepsilon_{j}(z)$, where $\varepsilon_{j}(z) \underset{z \rightarrow 1^{-}}{=} \mathcal{O}(\sqrt[2 N]{1-z})$ for any $j \in\{1, \ldots, N\}$. Putting this into (129), we get that

$$
\sum_{\ell=b}^{b+N-1}\left(1-\varepsilon_{j}(z)\right)^{\ell-b} H_{b, \ell}^{+}(z)=\left(1-\varepsilon_{j}(z)\right)^{-b} ;
$$

that is,

$$
\begin{aligned}
\sum_{k=0}^{N-1}(-1)^{k}\left(\sum_{\ell=b+k}^{b+N-1}\left(\begin{array}{c}
\ell-b \\
k
\end{array}\right) H_{b, \ell}^{+}(z)\right) \varepsilon_{j}(z)^{k} \\
=\sum_{k=0}^{\infty}(-1)^{k}\left(\begin{array}{c}
-b \\
k
\end{array}\right) \varepsilon_{j}(z)^{k} \\
=\sum_{k=0}^{\infty}\left(\begin{array}{c}
b+k-1 \\
b-1
\end{array}\right) \varepsilon_{j}(z)^{k} .
\end{aligned}
$$

Set

$$
\begin{gathered}
M_{k}(z)=(-1)^{k} \sum_{\ell=b+k}^{b+N-1}\left(\begin{array}{c}
\ell-b \\
k
\end{array}\right) H_{b, \ell}^{+}(z)-\left(\begin{array}{c}
b+k-1 \\
b-1
\end{array}\right), \\
R_{j}(z)=\sum_{\ell=N}^{\infty}\left(\begin{array}{c}
b+\ell-1 \\
b-1
\end{array}\right) \varepsilon_{j}(z)^{\ell} .
\end{gathered}
$$

Then, equality (154) reads

$$
\sum_{k=0}^{N-1} M_{k}(z) \varepsilon_{j}(z)^{k}=R_{j}(z), \quad 1 \leq j \leq N .
$$

This is a Vandermonde system, the solution of which is given by $M_{k}(z)=\widetilde{V}_{k}(z) / \widetilde{V}(z), 0 \leq k \leq N-1$, where

$$
\widetilde{V}(z)=\left|\begin{array}{cccc}
1 & \varepsilon_{1}(z) & \cdots & \varepsilon_{1}(z)^{N-1} \\
1 & \varepsilon_{2}(z) & \cdots & \varepsilon_{2}(z)^{N-1} \\
\vdots & \vdots & & \vdots \\
1 & \varepsilon_{N}(z) & \cdots & \varepsilon_{N}(z)^{N-1}
\end{array}\right|
$$

$\widetilde{V}_{k}(z)$

$$
=\left|\begin{array}{cccccccc}
1 & \varepsilon_{1}(z) & \cdots & \varepsilon_{1}(z)^{k-1} & R_{1}(z) & \varepsilon_{1}(z)^{k+1} & \cdots & \varepsilon_{1}(z)^{N-1} \\
1 & \varepsilon_{2}(z) & \cdots & \varepsilon_{2}(z)^{k-1} & R_{2}(z) & \varepsilon_{2}(z)^{k+1} & \cdots & \varepsilon_{2}(z)^{N-1} \\
\vdots & \vdots & & \vdots & \vdots & \vdots & & \vdots \\
1 & \varepsilon_{N}(z) & \cdots & \varepsilon_{N}(z)^{k-1} & R_{N}(z) & \varepsilon_{N}(z)^{k+1} & \cdots & \varepsilon_{N}(z)^{N-1}
\end{array}\right| .
$$

Since, by (85), $\varepsilon_{j}(z) \underset{z \rightarrow 1^{-}}{\sim}$ constant $\times \sqrt[2 N]{1-z}$ for any $j \epsilon$ $\{1, \ldots, N\}$, we have that

$$
\begin{aligned}
\widetilde{V}(z) & =\prod_{1 \leq \ell<m \leq N}\left[\varepsilon_{m}(z)-\varepsilon_{\ell}(z)\right] \\
& \underset{z \rightarrow 1^{-}}{\sim} \operatorname{constant} \times(1-z)^{(N-1) / 4}
\end{aligned}
$$

and second,

$$
R_{j}(z) \underset{z \rightarrow 1^{-}}{\sim}\left(\begin{array}{c}
b+N-1 \\
b-1
\end{array}\right) \varepsilon_{j}(z)^{N} \sim \text { constant } \times \sqrt{1-z}
$$

which implies, for $k \in\{0, \ldots, N-1\}$, that

$$
\begin{aligned}
\widetilde{V}_{k}(z) \underset{z}{z}=1^{-} & \mathcal{O}\left[(1-z)^{1 / 2+\left(\sum_{1 \leq m \leq N-1, m \neq k} m\right) /(2 N)}\right] \\
& =o\left[(1-z)^{(N-1) / 4}\right] .
\end{aligned}
$$

Therefore, $\lim _{z \rightarrow 1^{-}} M_{k}(z)=0$. On the other hand, for $z \in$ $(0,1)$, referring to the definition of $M_{k}(z)$, we can see that the quantity $H_{b, \ell}^{+}(z)$ can be expressed as a linear combination of

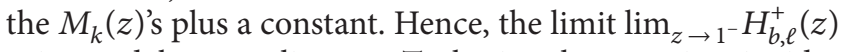
exists and, by appealing to a Tauberian theorem, it coincides with $H_{b, \ell}^{+}(1)$. This finishes the proof of (152).

Theorem 22. The pseudodistribution of $S_{b}^{+}$is characterized by the following pseudo-probabilities: for any $\ell \in\{b, b+1, \ldots, b+$ $N-1\}$,

$$
\mathbb{P}\left\{S_{b}^{+}=\ell, \sigma_{b}^{+}<+\infty\right\}=(-1)^{b+\ell} \frac{b}{\ell}\left(\begin{array}{c}
N-1 \\
\ell-b
\end{array}\right)\left(\begin{array}{c}
b+N-1 \\
b
\end{array}\right) .
$$

Moreover, $\mathbb{P}\left\{\sigma_{b}^{+}<+\infty\right\}=1$.

Proof. We explicitly solve system (152) rewritten as

$$
\sum_{\ell=k}^{N-1}\left(\begin{array}{l}
\ell \\
k
\end{array}\right) H_{b, \ell+b}^{+}(1)=(-1)^{k}\left(\begin{array}{c}
b+k-1 \\
b-1
\end{array}\right), \quad 0 \leq k \leq N-1 .
$$

The matrix of the system is $\left(\left(\begin{array}{l}\ell \\ k\end{array}\right)\right)_{0 \leq k, \ell \leq N-1}$ which admits $\left((-1)^{k+\ell}\left(\begin{array}{l}\ell \\ k\end{array}\right)\right)_{0 \leq k, \ell \leq N-1}$ as an inverse with the convention of settings $\left(\begin{array}{l}\ell \\ k\end{array}\right)=0$ if $k>\ell$. The solution of the system is given, for $\ell \in\{0,1, \ldots, N-1\}$, by

$$
\begin{aligned}
H_{b, \ell+b}^{+}(1) & =\sum_{k=\ell}^{N-1}(-1)^{k+\ell}\left(\begin{array}{l}
k \\
\ell
\end{array}\right) \times(-1)^{k}\left(\begin{array}{c}
b+k-1 \\
b-1
\end{array}\right) \\
& =(-1)^{\ell}\left(\begin{array}{c}
b+\ell-1 \\
b-1
\end{array}\right) \sum_{k=\ell}^{N-1}\left(\begin{array}{c}
b+k-1 \\
b+\ell-1
\end{array}\right) \\
& =(-1)^{\ell}\left(\begin{array}{c}
b+\ell-1 \\
b-1
\end{array}\right)\left(\begin{array}{c}
b+N-1 \\
b+\ell
\end{array}\right) \\
& =(-1)^{\ell} \frac{b}{\ell+b}\left(\begin{array}{c}
N-1 \\
\ell
\end{array}\right)\left(\begin{array}{c}
b+N-1 \\
b
\end{array}\right) .
\end{aligned}
$$

This proves (161). Now, by summing the $\mathbb{P}\left\{S_{b}^{+}=\ell, \sigma_{b}^{+}<+\infty\right\}$, $b \leq \ell \leq b+N-1$, given by (161), we obtain that

$$
\begin{aligned}
\mathbb{P}\left\{\sigma_{b}^{+}<+\infty\right\} & =(-1)^{b} b\left(\begin{array}{c}
b+N-1 \\
b
\end{array}\right) \sum_{\ell=b}^{b+N-1} \frac{(-1)^{\ell}}{\ell}\left(\begin{array}{c}
N-1 \\
\ell-b
\end{array}\right) \\
& =b\left(\begin{array}{c}
b+N-1 \\
b
\end{array}\right) \sum_{\ell=0}^{N-1} \frac{(-1)^{\ell}}{\ell+b}\left(\begin{array}{c}
N-1 \\
\ell
\end{array}\right) .
\end{aligned}
$$


Writing $1 /(\ell+b)=\int_{0}^{1} x^{\ell+b-1} \mathrm{~d} x$, we see that

$$
\begin{aligned}
& \sum_{\ell=0}^{N-1} \frac{(-1)^{\ell}}{\ell+b}\left(\begin{array}{c}
N-1 \\
\ell
\end{array}\right) \\
& =\int_{0}^{1}\left(\sum_{\ell=0}^{N-1}(-1)^{\ell}\left(\begin{array}{c}
N-1 \\
\ell
\end{array}\right) x^{\ell}\right) x^{b-1} \mathrm{~d} x \\
& =\int_{0}^{1}(1-x)^{N-1} x^{b-1} \mathrm{~d} x=\frac{(N-1) !(b-1) !}{(b+N-1) !} .
\end{aligned}
$$

Hence $\mathbb{P}\left\{\sigma_{b}^{+}<+\infty\right\}=1$. The proof of Theorem 22 is finished.

In the sequel, when considering $S_{b}^{+}$, we will omit the condition $\sigma_{b}^{+}<+\infty$.

Example 23. Let us have a look on the particular values $1,2,3,4$ of $N$.

(i) Case $N=1$. Evidently, in this case $S_{b}^{+}=b$ and then

$$
\mathbb{P}\left\{S_{b}^{+}=b\right\}=1 \text {. }
$$

This is the case of the ordinary random walk!

(ii) Case $N=2$. In this case the pseudorandom variables $\xi_{n}, n \in \mathbb{N}^{*}$, have two-valued upward jumps. Then the overshooting place must be either $b$ or $b+1: S_{b}^{+} \in\{b, b+1\}$. We have that

$$
\mathbb{P}\left\{S_{b}^{+}=b\right\}=b+1, \quad \mathbb{P}\left\{S_{b}^{+}=b+1\right\}=-b .
$$

Of course, we immediately see that $\mathbb{P}\left\{S_{b}^{+}=b\right\}+\mathbb{P}\left\{S_{b}^{+}=\right.$ $b+1\}=1$.

(iii) Case $N=3$. In this case $S_{b}^{+} \in\{b, b+1, b+2\}$ and

$$
\begin{gathered}
\mathbb{P}\left\{S_{b}^{+}=b\right\}=\frac{1}{2}(b+1)(b+2), \\
\mathbb{P}\left\{S_{b}^{+}=b+1\right\}=-b(b+2), \\
\mathbb{P}\left\{S_{b}^{+}=b+2\right\}=\frac{1}{2} b(b+1) .
\end{gathered}
$$

We can easily check that $\mathbb{P}\left\{S_{b}^{+}=b\right\}+\mathbb{P}\left\{S_{b}^{+}=b+1\right\}+\mathbb{P}\left\{S_{b}^{+}=\right.$ $b+2\}=1$.

(iv) Case $N=4$. In this case $S_{b}^{+} \in\{b, b+1, b+2, b+3\}$ and

$$
\begin{gathered}
\mathbb{P}\left\{S_{b}^{+}=b\right\}=\frac{1}{6}(b+1)(b+2)(b+3), \\
\mathbb{P}\left\{S_{b}^{+}=b+1\right\}=-\frac{1}{2} b(b+2)(b+3), \\
\mathbb{P}\left\{S_{b}^{+}=b+2\right\}=\frac{1}{2} b(b+1)(b+3), \\
\mathbb{P}\left\{S_{b}^{+}=b+3\right\}=-\frac{1}{6} b(b+1)(b+2) .
\end{gathered}
$$

We can easily check that $\mathbb{P}\left\{S_{b}^{+}=b\right\}+\mathbb{P}\left\{S_{b}^{+}=b+1\right\}+\mathbb{P}\left\{S_{b}^{+}=\right.$ $b+2\}+\mathbb{P}\left\{S_{b}^{+}=b+3\right\}=1$.
3.1.3. Pseudomoments of $S_{b}^{+}$. In the sequel, we use the notation $(i)_{n}=i(i-1)(i-2) \cdots(i-n+1)$ for any $i \in \mathbb{Z}$ and any $n \in \mathbb{N}^{*}$ and $(i)_{0}=1$. Of course, $(i)_{n}=i ! /(i-n) !$ and $(i)_{n} / n !=\left(\begin{array}{l}i \\ n\end{array}\right)$ if $i \geq n$. We also use the conventions $1 / i !=0$ for any negative integer $i$ and $\sum_{k=i}^{j}=0$ if $i>j$.

In this section, we compute several functionals related to the pseudomoments of $S_{b}^{+}$. More precisely, we provide formulae for $\mathbb{E}\left[\left(S_{b}^{+}-\beta\right)_{n}\right]$ (Theorem 25), $\mathbb{E}\left[\left(S_{b}^{+}-b\right)_{n}\right]$ (Corollary 26), $\mathbb{E}\left[\left(S_{b}^{+}\right)_{n}\right]$, and $\mathbb{E}\left[\left(S_{b}^{+}\right)^{n}\right]$ (Theorem 27).

Putting the elementary identity $1 /(\ell+b)=\int_{0}^{1} x^{\ell+b-1} \mathrm{~d} x$ into the equality

$$
\begin{aligned}
\mathbb{E}\left[f\left(S_{b}^{+}\right)\right] & =\left(\begin{array}{c}
b+N-1 \\
b
\end{array}\right) \sum_{\ell=b}^{b+N-1}(-1)^{b+\ell} \frac{b}{\ell}\left(\begin{array}{c}
N-1 \\
\ell-b
\end{array}\right) f(\ell) \\
& =b\left(\begin{array}{c}
b+N-1 \\
b
\end{array}\right) \sum_{\ell=0}^{N-1}(-1)^{\ell}\left(\begin{array}{c}
N-1 \\
\ell
\end{array}\right) \frac{f(\ell+b)}{\ell+b}
\end{aligned}
$$

we get the following integral representation of $\mathbb{E}\left[f\left(S_{b}^{+}\right)\right]$.

Theorem 24. For any function $f$ defined on $\{b, \ldots, b+N-1\}$,

$$
\begin{aligned}
\mathbb{E}\left[f\left(S_{b}^{+}\right)\right]= & b\left(\begin{array}{c}
b+N-1 \\
b
\end{array}\right) \\
& \times \int_{0}^{1}\left(\sum_{\ell=0}^{N-1}(-1)^{\ell}\left(\begin{array}{c}
N-1 \\
\ell
\end{array}\right) f(\ell+b) x^{\ell}\right) x^{b-1} \mathrm{~d} x .
\end{aligned}
$$

Theorem 25. For any integers $n \geq 0$ and $\beta$, the factorial pseudo-moment of $\left(S_{b}^{+}-\beta\right)$ of order $n$ is given by

$$
\begin{aligned}
& \mathbb{E}\left[\left(S_{b}^{+}-\beta\right)_{n}\right] \\
& =\left\{\begin{aligned}
& \frac{(b-\beta) !}{(b-1) !} \sum_{k=0 \vee(n+\beta-b)}^{n \wedge(N-1)}(-1)^{k} \frac{(k+b-1) !}{(k+b-\beta-n) !}\left(\begin{array}{l}
n \\
k
\end{array}\right) \text { if } \beta \leq b, \\
& \frac{(-1)^{n}}{(b-1) !(\beta-b-1) !} \\
& \times \sum_{k=0}^{n \wedge(N-1)}(k+b-1) !(\beta-b+n-k-1) !\left(\begin{array}{l}
n \\
k
\end{array}\right) \\
& \text { if } \beta \geq b+1 .
\end{aligned}\right.
\end{aligned}
$$

If $n \leq N-1$, we simply have that

$$
\mathbb{E}\left[\left(S_{b}^{+}-\beta\right)_{n}\right]=(-\beta)_{n}=(-1)^{n} \beta(\beta+1) \cdots(\beta+n-1) .
$$


Proof. By (171), we have that

$$
\begin{aligned}
\mathbb{E}\left[\left(S_{b}^{+}-\beta\right)_{n}\right] \\
=b\left(\begin{array}{c}
b+N-1 \\
b
\end{array}\right) \\
\quad \times \int_{0}^{1}\left(\sum_{\ell=0}^{N-1}(-1)^{\ell}\left(\begin{array}{c}
N-1 \\
\ell
\end{array}\right)(\ell+b-\beta)_{n} x^{\ell+b-1}\right) \mathrm{d} x .
\end{aligned}
$$

Next, by observing that $(\ell+b-\beta)_{n} x^{\ell+b-1}=x^{n+\beta-1} \times$ $\left(\mathrm{d}^{n} / \mathrm{d} x^{n}\right)\left(x^{\ell+b-\beta}\right)$, we obtain that

$$
\begin{aligned}
\sum_{\ell=0}^{N-1}(-1)^{\ell}\left(\begin{array}{c}
N-1 \\
\ell
\end{array}\right)(\ell+b-\beta)_{n} x^{\ell+b-1} \\
=\sum_{\ell=0}^{N-1}\left((-1)^{\ell}\left(\begin{array}{c}
N-1 \\
\ell
\end{array}\right) \frac{\mathrm{d}^{n}}{\mathrm{~d} x^{n}}\left(x^{\ell+b-\beta}\right)\right) x^{n+\beta-1} \\
=\frac{\mathrm{d}^{n}}{\mathrm{~d} x^{n}}\left(\sum_{\ell=0}^{N-1}(-1)^{\ell}\left(\begin{array}{c}
N-1 \\
\ell
\end{array}\right) x^{\ell+b-\beta}\right) x^{n+\beta-1} \\
=\frac{\mathrm{d}^{n}}{\mathrm{~d} x^{n}}\left((1-x)^{N-1} x^{b-\beta}\right) x^{n+\beta-1} .
\end{aligned}
$$

Applying Leibniz rule to (175), we see that

$$
\begin{aligned}
& \sum_{\ell=0}^{N-1}(-1)^{\ell}\left(\begin{array}{c}
N-1 \\
\ell
\end{array}\right)(\ell+b-\beta)_{n} x^{\ell+b-1} \\
& =\left(\sum_{k=0}^{n}\left(\begin{array}{l}
n \\
k
\end{array}\right) \frac{\mathrm{d}^{k}}{\mathrm{~d} x^{k}}\left((1-x)^{N-1}\right) \frac{\mathrm{d}^{n-k}}{\mathrm{~d} x^{n-k}}\left(x^{b-\beta}\right)\right) x^{n+\beta-1} \\
& =\sum_{k=0}^{n \wedge(N-1)}(-1)^{k}\left(\begin{array}{l}
n \\
k
\end{array}\right)(N-1)_{k}(b-\beta)_{n-k} \\
& \quad \times(1-x)^{N-1-k} x^{k+b-1} .
\end{aligned}
$$

Therefore,

$$
\begin{gathered}
\int_{0}^{1}\left(\sum_{\ell=0}^{N-1}(-1)^{\ell}\left(\begin{array}{c}
N-1 \\
\ell
\end{array}\right)(\ell+b-\beta)_{n} x^{\ell+b-1}\right) \mathrm{d} x \\
=\sum_{k=0}^{n \wedge(N-1)}(-1)^{k}(N-1)_{k}(b-\beta)_{n-k}\left(\begin{array}{l}
n \\
k
\end{array}\right) \\
\quad \times \int_{0}^{1}(1-x)^{N-1-k} x^{k+b-1} \mathrm{~d} x \\
=\sum_{k=0}^{n \wedge(N-1)}(-1)^{k}(N-1)_{k}(b-\beta)_{n-k}\left(\begin{array}{l}
n \\
k
\end{array}\right) \\
\times \frac{(N-1-k) !(k+b-1) !}{(b+N-1) !}
\end{gathered}
$$

$$
\left\{\begin{array}{l}
\frac{(b-\beta) !(N-1) !}{(b+N-1) !} \\
\times \sum_{k=0 \vee(n+\beta-b)}^{n \wedge(N-1)}(-1)^{k} \frac{(k+b-1) !}{(k+b-\beta-n) !}\left(\begin{array}{l}
n \\
k
\end{array}\right) \\
\frac{(-1)^{n}(N-1) !}{(\beta-b-1) !(b+N-1) !} \\
\times \sum_{k=0}^{n \wedge(N-1)}(k+b-1) !(\beta-b+n-k-1) !\left(\begin{array}{l}
n \\
k
\end{array}\right) \\
\text { if } \beta \leq b \geq b+1 .
\end{array}\right.
$$

Finally, plugging (177) into (175) and (174) yields (172).

Assume now that $n \leq N-1$ and $\beta \leq b$. If $n \geq 1-\beta$, we can write in (172) that

$$
\frac{(k+b-1) !}{(k+b-\beta-n) !}=\left.\frac{\mathrm{d}^{\beta+n-1}}{\mathrm{~d} x^{\beta+n-1}}\left(x^{k+b-1}\right)\right|_{x=1} .
$$

Then,

$$
\begin{aligned}
& \sum_{k=0 \vee(n+\beta-b)}^{n \wedge(N-1)}(-1)^{k} \frac{(k+b-1) !}{(k+b-\beta-n) !}\left(\begin{array}{l}
n \\
k
\end{array}\right) \\
& \quad=\sum_{k=0}^{n}(-1)^{k} \frac{(k+b-1) !}{(k+b-\beta-n) !}\left(\begin{array}{l}
n \\
k
\end{array}\right) \\
& =\left.\frac{\mathrm{d}^{\beta+n-1}}{\mathrm{~d} x^{\beta+n-1}}\left[\left(\sum_{k=0}^{n}(-1)^{k}\left(\begin{array}{l}
n \\
k
\end{array}\right) x^{k}\right) x^{b-1}\right]\right|_{x=1} \\
& =\left.\frac{\mathrm{d}^{\beta+n-1}}{\mathrm{~d} x^{\beta+n-1}}\left((1-x)^{n} x^{b-1}\right)\right|_{x=1} \\
& =\sum_{k=0}^{n}(-1)^{k}(n)_{k}(b-1)_{\beta+n-k-1}\left(\begin{array}{c}
k+n-1 \\
k
\end{array}\right) \\
& =(-1)^{n} \frac{(b-1) !}{(b-\beta) !} \beta(\beta+1) \cdots(\beta+n-1) . \\
& =(-1)^{n}(n)_{n}(b-1)_{\beta-1}\left(\begin{array}{c}
(b+1) !(\beta+n-1) ! \\
(b-\beta) !(\beta-1) !
\end{array}\right. \\
& \left.\times(1-x)^{n-k} x^{k+b-\beta-n}\right)\left.\right|_{x=1} \\
& =((b-1) \\
& =
\end{aligned}
$$

Putting (179) into (172) yields (173). If $n \leq-\beta$ (which requires that $\beta \leq 0)$, in (172), we write instead that

$$
\frac{(k+b-1) !}{(k+b-\beta-n) !}=\frac{1}{(-n-\beta) !} \int_{0}^{1} x^{k+b-1}(1-x)^{-n-\beta} \mathrm{d} x .
$$


Then,

$$
\begin{aligned}
\sum_{k=0 \vee(n+\beta-b)}^{n \wedge(N-1)}(-1)^{k} \frac{(k+b-1) !}{(k+b-\beta-n) !}\left(\begin{array}{l}
n \\
k
\end{array}\right) & \\
= & \sum_{k=0}^{n}(-1)^{k} \frac{(k+b-1) !}{(k+b-\beta-n) !}\left(\begin{array}{l}
n \\
k
\end{array}\right) \\
= & \frac{1}{(-n-\beta) !} \\
& \times \int_{0}^{1}\left(\sum_{k=0}^{n}(-1)^{k}\left(\begin{array}{l}
n \\
k
\end{array}\right) x^{k}\right) x^{b-1}(1-x)^{-n-\beta} \mathrm{d} x \\
= & \frac{1}{(-n-\beta) !} \int_{0}^{1} x^{b-1}(1-x)^{-\beta} \mathrm{d} x \\
= & \frac{(b-1) !(-\beta) !}{(b-\beta) !(-n-\beta) !} \\
= & (-1)^{n} \frac{(b-1) !}{(b-\beta) !} \beta(\beta+1) \cdots(\beta+n-1) .
\end{aligned}
$$

Putting (181) into (172) yields (173) in this case too.

Assume finally that $n \leq N-1$ and $\beta \geq b+1$. We write in (172) that

$$
\begin{aligned}
& (k+b-1) !(\beta-b+n-k-1) ! \\
& \quad=(\beta+n-1) ! \int_{0}^{1} x^{k+b-1}(1-x)^{\beta-b+n-k-1} \mathrm{~d} x .
\end{aligned}
$$

Then

$$
\begin{aligned}
\sum_{k=0}^{n \wedge(N-1)}(k+b-1) !(\beta-b+n-k-1) !\left(\begin{array}{l}
n \\
k
\end{array}\right) \\
=(\beta+n-1) ! \\
\quad \times \int_{0}^{1}\left[\sum_{k=0}^{n}\left(\begin{array}{l}
n \\
k
\end{array}\right)\left(\frac{x}{1-x}\right)^{k}\right] x^{b-1}(1-x)^{\beta-b+n-1} \mathrm{~d} x \\
=(\beta+n-1) ! \int_{0}^{1} x^{b-1}(1-x)^{\beta-b-1} \mathrm{~d} x \\
=(b-1) !(\beta-b-1) ! \frac{(\beta+n-1) !}{(\beta-1) !} .
\end{aligned}
$$

Putting (183) into (172) yields (173).

By choosing $\beta=b$ in Theorem 25, we derive that

$$
\mathbb{E}\left[\left(S_{b}^{+}-b\right)_{n}\right]=\frac{1}{(b-1) !} \sum_{k=n}^{n \wedge(N-1)}(-1)^{k} \frac{(k+b-1) !}{(k-n) !}\left(\begin{array}{l}
n \\
k
\end{array}\right) .
$$

We immediately obtain the following particular result which will be used in Theorem 28 .

Corollary 26. The factorial pseudomoments of $\left(S_{b}^{+}-b\right)$ are given by

$$
\mathbb{E}\left[\left(S_{b}^{+}-b\right)_{n}\right]= \begin{cases}(-b)_{n} & \text { if } 0 \leq n \leq N-1 \\ 0 & \text { if } n \geq N\end{cases}
$$

The above identity can be rewritten, if $0 \leq n \leq N-1$, as

$$
\mathbb{E}\left[\left(\begin{array}{c}
S_{b}^{+}-b \\
n
\end{array}\right)\right]=(-1)^{n}\left(\begin{array}{c}
n+b-1 \\
b-1
\end{array}\right) .
$$

Moreover, since $S_{b}^{+} \in\{b, b+1, \ldots, b+N-1\}$, it is clear that $\left(S_{b}^{+}-b\right)\left(S_{b}^{+}-b-1\right) \cdots\left(S_{b}^{+}-b-N+1\right)=0$ which immediately entails that $\left(S_{b}^{+}-b\right)_{n}=0$ for any $n \geq N$; then $\mathbb{E}\left[\left(S_{b}^{+}-b\right)_{n}\right]=0$ for $n \geq N$ as stated in Corollary 26 .

By choosing $\beta=0$ in Theorem 25, we plainly extract that $\mathbb{E}\left[\left(S_{b}^{+}\right)_{n}\right]=0$ for $n \in\{1, \ldots, N-1\}$. Moreover, as previously mentioned, $\left(S_{b}^{+}\right)_{n}=0$ for any $n \geq b+N$; then $\mathbb{E}\left[\left(S_{b}^{+}\right)_{n}\right]=$ 0 for $n \geq b+N$. Actually, we can compute the factorial pseudomoments of $S_{b}^{+}, \mathbb{E}\left[\left(S_{b}^{+}\right)_{n}\right]$, for $n \in\{N, N+1, \ldots, b+N\}$. The formula of Theorem 25 seems to be untractable, so we provide another way for evaluating them.

Theorem 27. The factorial pseudomoments of $S_{b}^{+}$are given by

$$
\mathbb{E}\left[\left(S_{b}^{+}\right)_{n}\right]=\left\{\begin{array}{c}
(-1)^{N-1}\left(\begin{array}{c}
n-1 \\
N-1
\end{array}\right)(b+N-1)_{n} \\
\text { if } N \leq n \leq b+N-1, \\
0 \quad \text { if } 1 \leq n \leq N-1 \text { or } n \geq b+N .
\end{array}\right.
$$

Moreover, for $n \in\{1, \ldots, N-1\}$, the pseudo-moment of $S_{b}^{+}$of order $n$ vanishes:

$$
\begin{gathered}
\mathbb{E}\left[\left(S_{b}^{+}\right)^{n}\right]=0, \\
\mathbb{E}\left[\left(S_{b}^{+}\right)^{N}\right]=(-1)^{N-1} \frac{(b+N-1) !}{(b-1) !}=-(-b)_{N} .
\end{gathered}
$$

Proof. We focus on the case where $N \leq n \leq b+N-1$. We have that

$$
\begin{aligned}
\mathbb{E}\left[\left(S_{b}^{+}\right)_{n}\right] & =\sum_{\ell=0}^{N-1} \mathbb{P}\left\{S_{b}^{+}=\ell+b\right\}(\ell+b)_{n} \\
& =b\left(\begin{array}{c}
b+N-1 \\
b
\end{array}\right) \sum_{\ell=0}^{N-1}(-1)^{\ell}(\ell+b-1)_{n-1}\left(\begin{array}{c}
N-1 \\
\ell
\end{array}\right) .
\end{aligned}
$$


The intermediate sum lying in the last displayed equality can be evaluated as follows: by observing that $(\ell+b-1)_{n-1}=$ $\left.\left(\mathrm{d}^{n-1} / \mathrm{d} x^{n-1}\right)\left(x^{\ell+b-1}\right)\right|_{x=1}$ and appealing to Leibniz rule, we obtain that

$$
\begin{aligned}
& \sum_{\ell=0}^{N-1}(-1)^{\ell}(\ell+b-1)_{n-1}\left(\begin{array}{c}
N-1 \\
\ell
\end{array}\right) \\
& =\left.\frac{\mathrm{d}^{n-1}}{\mathrm{~d} x^{n-1}}\left(\sum_{\ell=0}^{N-1}(-1)^{\ell}\left(\begin{array}{c}
N-1 \\
\ell
\end{array}\right) x^{\ell+b-1}\right)\right|_{x=1} \\
& =\left.\frac{\mathrm{d}^{n-1}}{\mathrm{~d} x^{n-1}}\left((1-x)^{N-1} x^{b-1}\right)\right|_{x=1} \\
& =\left.\left.\sum_{j=N-1}^{n-1}\left(\begin{array}{c}
n-1 \\
j
\end{array}\right) \frac{\mathrm{d}^{j}}{\mathrm{~d} x^{j}}\left((1-x)^{N-1}\right)\right|_{x=1} \frac{\mathrm{d}^{n-1-j}}{\mathrm{~d} x^{n-1-j}}\left(x^{b-1}\right)\right|_{x=1} \\
& =(-1)^{N-1}(N-1) !\left(\begin{array}{c}
n-1 \\
N-1
\end{array}\right)(b-1)_{n-N} \\
& =(-1)^{N-1} \frac{(b-1) !(n-1) !}{(n-N) !(b+N-n-1) !} .
\end{aligned}
$$

Consequently,

$$
\begin{aligned}
\mathbb{E}\left[\left(S_{b}^{+}\right)_{n}\right] & =(-1)^{N-1} b\left(\begin{array}{c}
b+N-1 \\
b
\end{array}\right) \frac{(b-1) !(n-1) !}{(n-N) !(b+N-n-1) !} \\
& =(-1)^{N-1} \frac{(n-1) !(b+N-1) !}{(n-N) !(N-1) !(b+N-n-1) !} .
\end{aligned}
$$

This is the result announced in Theorem 27 when $N \leq n \leq$ $b+N-1$.

Next, concerning the pseudomoments of $S_{b}^{+}$, we appeal to an elementary argument of linear algebra: the family $\left(1, X_{1}, X_{2}, \ldots, X_{n}\right)$ (recall that $X_{k}=X(X-1) \cdots(X-$ $k+1)$ ) is a basis of the space of polynomials of degree not greater than $n$. So, $X^{n}$ can be written as a linear combination of $X_{1}, X_{2}, \ldots, X_{n}$. Then $\mathbb{E}\left[\left(S_{b}^{+}\right)^{n}\right]$ can be written as a linear combination of the factorial pseudomoments of $S_{b}^{+}$of order between 1 and $n$. The latters cancel for $n \in\{1, \ldots, N-1\}$. As a result, $\mathbb{E}\left[\left(S_{b}^{+}\right)^{n}\right]=0$.

The same argument ensures the equalities $\mathbb{E}\left[\left(S_{b}^{+}\right)_{N}\right]=$ $\mathbb{E}\left[\left(S_{b}^{+}\right)^{N}\right]$, which is equal to $(-1)^{N-1}(b+N-1)_{N}$, and $\mathbb{E}\left[\left(S_{b}^{+}-b\right)_{N}\right]=\mathbb{E}\left[\left(S_{b}^{+}\right)^{N}\right]+(-b)^{N}$ which vanishes. Each of them yields the value of $\mathbb{E}\left[\left(S_{b}^{+}\right)^{N}\right]$.

The proof of Theorem 27 is completed.

3.2. Link with the High-Order Finite-Difference Operator. Set $\Delta^{+} f(i)=f(i+1)-f(i)$ for any $i \in \mathbb{Z}$ and $\left(\Delta^{+}\right)^{j}=\underbrace{\Delta^{+} \circ \cdots \circ \Delta^{+}}_{j \text { times }}$ for any $j \in \mathbb{N}^{*}$. Set also $\left(\Delta^{+}\right)^{0} f=f$. The quantity $\left(\Delta^{+}\right)^{j}$ is the iterated forward finite-difference operator given by

$$
\left(\Delta^{+}\right)^{j} f(i)=\sum_{k=0}^{j}(-1)^{j+k}\left(\begin{array}{l}
j \\
k
\end{array}\right) f(i+k) .
$$

Conversely, $f(i+k)$ can be expressed by means of $\left(\Delta^{+}\right)^{j} f(i)$, $0 \leq j \leq k$, according to

$$
f(i+k)=\sum_{j=0}^{k}\left(\begin{array}{l}
k \\
j
\end{array}\right)\left(\Delta^{+}\right)^{j} f(i) .
$$

We have the following expression for any functional of the pseudorandom variable $S_{b}^{+}$.

Theorem 28. One has, for any function $f$ defined on $\{b, b+$ $1, \ldots, b+N-1\}$, that

$$
\mathbb{E}\left[f\left(S_{b}^{+}\right)\right]=\sum_{j=0}^{N-1}(-1)^{j}\left(\begin{array}{c}
j+b-1 \\
b-1
\end{array}\right)\left(\Delta^{+}\right)^{j} f(b) .
$$

Proof. By (193), we see that

$$
\begin{aligned}
\mathbb{E}\left[f\left(S_{b}^{+}\right)\right] & =\mathbb{E}\left(\sum_{j=0}^{S_{b}^{+}-b}\left(\begin{array}{c}
S_{b}^{+}-b \\
j
\end{array}\right)\left(\Delta^{+}\right)^{j} f(b)\right) \\
& =\sum_{j=0}^{N-1} \mathbb{E}\left[\left(\begin{array}{c}
S_{b}^{+}-b \\
j
\end{array}\right)\right]\left(\Delta^{+}\right)^{j} f(b)
\end{aligned}
$$

which immediately yields (194) thanks to (186).

Corollary 29. The generating function of $S_{b}^{+}$is given by

$$
\mathbb{E}\left(\zeta^{S_{b}^{+}}\right)=\zeta^{b} \sum_{j=0}^{N-1}\left(\begin{array}{c}
j+b-1 \\
b-1
\end{array}\right)(1-\zeta)^{j} .
$$

Proof. Let us apply Theorem 28 to the function $f(i)=\zeta^{i}$ for which we plainly have $\left(\Delta^{+}\right)^{j} f(i)=(-1)^{j} \zeta^{i}(1-\zeta)^{j}$. This immediately yields (196).

Remark 30. A direct computation with (161) yields the alternative representation:

$$
\mathbb{E}\left(\zeta^{S_{b}^{+}}\right)=b\left(\begin{array}{c}
b+N-1 \\
b
\end{array}\right) \zeta^{b} \int_{0}^{1} x^{b-1}(1-\zeta x)^{N-1} \mathrm{~d} x .
$$

Of special interest is the case when the starting point of the pseudorandom walk is any point $x \in \mathbb{Z}$. By translating $b$ into $b-x$ and the function $f$ into the shifted function $f(\cdot+x)$ in formula (194), we get that

$$
\begin{aligned}
& \mathbb{E}_{x}\left[f\left(S_{b}^{+}\right)\right] \stackrel{\text { def }}{=} \mathbb{E}\left[f\left(x+S_{b-x}^{+}\right)\right] \\
&=\sum_{j=0}^{N-1}(-1)^{j}\left(\begin{array}{c}
j+b-x-1 \\
j
\end{array}\right)\left(\Delta^{+}\right)^{j} f(b) .
\end{aligned}
$$

Thus, we obtain the following result.

Theorem 31. One has, for any function $f$ defined on $\{b, b+$ $1, \ldots, b+N-1\}$, that

$$
\mathbb{E}_{x}\left[f\left(S_{b}^{+}\right)\right]=\sum_{j=0}^{N-1} P_{b, j}^{+}(x)\left(\Delta^{+}\right)^{j} f(b)
$$


with $P_{b, 0}^{+}(x)=1$ and, for $j \in\{1, \ldots, N-1\}$,

$$
P_{b, j}^{+}(x)=\frac{1}{j !} \prod_{k=0}^{j-1}(x-b-k)
$$

The $P_{b, j}^{+}, 0 \leq j \leq N-1$, are Newton interpolating polynomials. They are of degree not greater than $(N-1)$ and characterized, for any $k \in\{0, \ldots, N-1\}$, by

$$
\left(\Delta^{+}\right)^{k} P_{b, j}^{+}(b)=\delta_{j k}
$$

Proof. Coming back to the proof of Theorem 28 and appealing to Theorem 22, we write that

$$
\begin{aligned}
P_{b, j}^{+}(x) & =\mathbb{E}_{x}\left[\left(\begin{array}{c}
S_{b}^{+}-b \\
j
\end{array}\right)\right] \\
& =\mathbb{E}\left[\left(\begin{array}{c}
S_{b-x}^{+}+x-b \\
j
\end{array}\right)\right] \\
& =\sum_{m=j}^{N-1}\left(\begin{array}{c}
m \\
j
\end{array}\right) \mathbb{P}\left\{S_{b-x}^{+}=b-x+m\right\} \\
& =\frac{1}{(N-1) !} \sum_{m=j}^{N-1}(-1)^{m}\left(\begin{array}{c}
m \\
j
\end{array}\right)\left(\begin{array}{c}
N-1 \\
m
\end{array}\right) K_{m}(x),
\end{aligned}
$$

where, for any $m \in\{0, \ldots, N-1\}$,

$$
K_{m}(x)=\frac{\left(\prod_{k=0}^{N-1}(b-x+k)\right)}{(b-x+m)}=\prod_{\substack{0 \leq k \leq N-1 \\ k \neq m}}(b-x+k) .
$$

The expression $K_{m}(x)$ defines a polynomial of the variable $x$ of degree $(N-1)$, so $P_{b, j}^{+}$is a polynomial of degree not greater than $(N-1)$. It is obvious that $K_{m}(b+\ell)=0$ for $\ell \in\{0, \ldots, N-$ $1\} \backslash\{m\}$. On the other hand, $K_{\ell}(b+\ell)=(-1)^{\ell}(N-1) ! /\left(\begin{array}{c}N-1 \\ \ell\end{array}\right)$. By putting this into (202), we get that

$$
P_{b, j}^{+}(b+\ell)=\left(\begin{array}{l}
\ell \\
j
\end{array}\right) \mathbb{1}_{\{j \leq \ell\}} .
$$

Next, we obtain, for any $k \in\{0, \ldots, N-1\}$, that

$$
\begin{aligned}
\left(\Delta^{+}\right)^{k} P_{b, j}^{+}(b) & =\sum_{\ell=0}^{k}(-1)^{k+\ell}\left(\begin{array}{l}
k \\
\ell
\end{array}\right) P_{b, j}^{+}(b+\ell) \\
& =\sum_{\ell=j}^{k}(-1)^{k+\ell}\left(\begin{array}{l}
k \\
\ell
\end{array}\right)\left(\begin{array}{l}
\ell \\
j
\end{array}\right) \\
& =(-1)^{j+k}\left(\begin{array}{c}
k \\
j
\end{array}\right) \sum_{\ell=0}^{k-j}(-1)^{\ell}\left(\begin{array}{c}
k-j \\
\ell
\end{array}\right)=\delta_{j k} .
\end{aligned}
$$

The proof of Theorem 31 is finished.

We complete this paragraph by stating a strong pseudoMarkov property related to time $\sigma_{b}^{+}$.
Theorem 32. One has, for any function $f$ defined on $\mathbb{Z}$ and any $n \in \mathbb{N}$, that

$$
\mathbb{E}_{x}\left[f\left(S_{\sigma_{b}^{+}+n}\right)\right]=\sum_{j=0}^{N-1} P_{b, j}^{+}(x)\left(\Delta^{+}\right)^{j} \mathbb{E}_{b}\left[f\left(S_{n}\right)\right] .
$$

In (206), the operator $\left(\Delta^{+}\right)^{j}$ acts on the variable $b$.

Proof. We denote by $\mathbb{P}_{x}$ the pseudoprobability associated with the pseudoexpectation $\mathbb{E}_{x}$. Actually, it represents the pseudoprobability related to the pseudorandom walk started at point $x$ at time 0 . We have, by independence of the $\xi_{j}$ 's, that

$$
\begin{aligned}
\mathbb{E}_{x} & {\left[f\left(S_{\sigma_{b}^{+}+n}\right)\right] } \\
= & \sum_{\substack{k, \ell \in \mathbb{N}: \\
b \leq \ell \leq b+N-1}} \mathbb{E}_{x}\left[\mathbb{1}_{\left\{\sigma_{b}^{+}=k, S_{b}^{+}=\ell\right\}} f\left(S_{b}^{+}+\xi_{k+1}+\cdots+\xi_{k+n}\right)\right] \\
= & \sum_{\substack{k, \ell \in \mathbb{N}: \\
b \leq \ell \leq b+N-1}} \mathbb{P}_{x}\left\{\sigma_{b}^{+}=k, S_{b}^{+}=\ell\right\} \mathbb{E}\left[f\left(\ell+\xi_{1}+\cdots+\xi_{n}\right)\right] \\
= & \sum_{\ell=b}^{b+N-1} \mathbb{P}_{x}\left\{S_{b}^{+}=\ell\right\} \mathbb{E}_{\ell}\left[f\left(S_{n}\right)\right] \\
= & \mathbb{E}_{x}\left[\mathbb{E}_{S_{b}^{+}}\left[f\left(S_{n}\right)\right]\right] .
\end{aligned}
$$

Hence, by setting $g(x)=\mathbb{E}_{x}\left[f\left(S_{n}\right)\right]$, we have obtained that

$$
\mathbb{E}_{x}\left[f\left(S_{\sigma_{b}^{+}+n}\right)\right]=\mathbb{E}_{x}\left[g\left(S_{b}^{+}\right)\right]
$$

which proves (206) thanks to (199).

Example 33. Below, we display the form of (206) for the particular values $1,2,3$ of $N$.

(i) For $N=1$, (206) reads

$$
\mathbb{E}_{x}\left[f\left(S_{\sigma_{b}^{+}+n}\right)\right]=\mathbb{E}_{b}\left[f\left(S_{n}\right)\right]
$$

which is of course trivial! This is the strong Markov property for the ordinary random walk.

(ii) For $N=2$, (206) reads

$$
\begin{aligned}
\mathbb{E}_{x}\left[f\left(S_{\sigma_{b}^{+}+n}\right)\right]= & \mathbb{E}_{b}\left[f\left(S_{n}\right)\right]+(x-b) \Delta^{+} \mathbb{E}_{b}\left[f\left(S_{n}\right)\right] \\
= & (b-x+1) \mathbb{E}_{b}\left[f\left(S_{n}\right)\right] \\
& +(x-b) \mathbb{E}_{b+1}\left[f\left(S_{n}\right)\right] .
\end{aligned}
$$


(iii) For $N=3$, (206) reads

$$
\begin{aligned}
\mathbb{E}_{x}\left[f\left(S_{\sigma_{b}^{+}+n}\right)\right]= & \mathbb{E}_{b}\left[f\left(S_{n}\right)\right]+(x-b) \Delta^{+} \mathbb{E}_{b}\left[f\left(S_{n}\right)\right] \\
& +\frac{1}{2}(x-b)(x-b-1)\left(\Delta^{+}\right)^{2} \mathbb{E}_{b}\left[f\left(S_{n}\right)\right] \\
= & \frac{1}{2}(x-b-1)(x-b-2) \mathbb{E}_{b}\left[f\left(S_{n}\right)\right] \\
& -(x-b)(x-b-2) \mathbb{E}_{b+1}\left[f\left(S_{n}\right)\right] \\
& +\frac{1}{2}(x-b)(x-b-1) \mathbb{E}_{b+2}\left[f\left(S_{n}\right)\right] .
\end{aligned}
$$

3.3. Joint Pseudodistribution of $\left(\tau_{b}^{+}, X_{b}^{+}\right)$. Below, we give an ad hoc definition for the convergence of a family of exit times.

Definition 34. Let $\left(\left(X_{t}^{\varepsilon}\right)_{t \geq 0}\right)_{\varepsilon>0}$ be a family of pseudoprocesses which converges towards a pseudoprocess $\left(X_{t}\right)_{t \geq 0}$ when $\varepsilon \rightarrow$ $0^{+}$in the sense of Definition 13. Let $I$ be a subset of $\mathbb{R}$ and set $\tau_{I}^{\varepsilon}=\inf \left\{t \geq 0: X_{t}^{\varepsilon} \notin I\right\}, X_{I}^{\varepsilon}=X_{\tau_{I}^{\varepsilon}}^{\varepsilon}$ and $\tau_{I}=\inf \left\{t \geq 0: X_{t} \notin\right.$ $I\}, X_{I}=X_{\tau_{I}}$.

We say that

$$
\left(\tau_{I}^{\varepsilon}, X_{I}^{\varepsilon}\right) \underset{\varepsilon \rightarrow 0^{+}}{\longrightarrow}\left(\tau_{I}, X_{I}\right)
$$

if and only if

$$
\begin{array}{r}
\forall \lambda>0, \quad \forall \mu \in \mathbb{R}, \\
\mathbb{E}\left(\mathrm{e}^{-\lambda \tau_{I}^{\varepsilon}+i \mu X_{I}^{\varepsilon}} \mathbb{1}_{\left\{\tau_{I}^{\varepsilon}<+\infty\right\}}\right) \underset{\varepsilon \rightarrow 0^{+}}{\longrightarrow} \mathbb{E}\left(\mathrm{e}^{-\lambda \tau_{I}+i \mu X_{I}} \mathbb{1}_{\left\{\tau_{I}<+\infty\right\}}\right) .
\end{array}
$$

We say that

$$
X_{I}^{\varepsilon} \underset{\varepsilon \rightarrow 0^{+}}{\longrightarrow} X_{I}
$$

if and only if

$$
\forall \mu \in \mathbb{R}, \quad \mathbb{E}\left(\mathrm{e}^{i \mu X_{I}^{\varepsilon}} \mathbb{1}_{\left\{\tau_{I}^{\varepsilon}<+\infty\right\}}\right) \underset{\varepsilon \rightarrow 0^{+}}{\longrightarrow} \mathbb{E}\left(\mathrm{e}^{i \mu X_{I}} \mathbb{1}_{\left\{\tau_{I}<+\infty\right\}}\right) .
$$

As in Section 2.3, we choose for the family $\left(\left(X_{t}^{\varepsilon}\right)_{t \geq 0}\right)_{\varepsilon>0}$ the pseudoprocesses defined, for any $\varepsilon>0$, by

$$
X_{t}^{\varepsilon}=\varepsilon S_{\left\lfloor t / \varepsilon^{2 N}\right\rfloor}, \quad t \geq 0,
$$

and for the pseudoprocess $\left(X_{t}\right)_{t>0}$ the pseudo-Brownian motion. For $I$, we choose the interval $(-\infty, b)$ so that $\tau_{I}^{\varepsilon}=\tau_{b}^{\varepsilon+}$, $X_{I}^{\varepsilon}=X_{b}^{\varepsilon+}$ and $\tau_{I}=\tau_{b}^{+}, X_{I}=X_{b}^{+}$. Set $b_{\varepsilon}=\lceil b / \varepsilon\rceil$ where $\lceil\cdot\rceil$ is the usual ceiling function. We have $\tau_{b}^{\varepsilon+}=\varepsilon^{2 N} \sigma_{b_{\varepsilon}^{+}}$and $X_{b}^{\varepsilon+}=\varepsilon S_{b_{\varepsilon}}^{+}$. Recall the setting $\varphi_{j}=-i \mathrm{e}^{i((2 j-1) / 2 N) \pi}, 1 \leq j \leq N$.

Theorem 35. Assume that $c \leq 1 / 2^{2 N-1}$. The following convergence holds:

$$
\left(\tau_{b}^{\varepsilon+}, X_{b}^{\varepsilon+}\right) \underset{\varepsilon \rightarrow 0^{+}}{\longrightarrow}\left(\tau_{b}^{+}, X_{b}^{+}\right)
$$

where, for any $\lambda>0$ and any $\mu \in \mathbb{R}$,

$$
\begin{aligned}
& \mathbb{E}\left(\mathrm{e}^{-\lambda \tau_{b}^{+}+i \mu X_{b}^{+}} \mathbb{1}_{\left\{\tau_{b}<+\infty\right\}}\right) \\
& =\mathrm{e}^{i \mu b} \sum_{k=1}^{N} \prod_{\substack{1 \leq j \leq N \\
j \neq k}} \frac{\varphi_{j}}{\varphi_{j}-\varphi_{k}} \prod_{\substack{1 \leq j \leq N \\
j \neq k}}\left(1-\frac{i \overline{\varphi_{j}} \mu}{\sqrt[2 N]{\lambda / c}}\right) \mathrm{e}^{-\varphi_{k} \sqrt[2 N]{\lambda / c} b} .
\end{aligned}
$$

Proof. We already pointed out that the assumption $c \leq$ $1 / 2^{2 N-1}$ entails that $M_{\infty}=1$. Therefore, (147) holds for $z=\mathrm{e}^{-\lambda \varepsilon^{2 N}}<1 / M_{\infty}=1$, that is, for $\lambda>0$. So, by (147), we have, for $\lambda>0$, that

$$
\begin{aligned}
\mathbb{E}\left(\mathrm{e}^{-\lambda \tau_{b}^{\varepsilon+}+i \mu X_{b}^{\varepsilon+}} \mathbb{1}_{\left\{\tau_{b}^{\varepsilon+}<+\infty\right\}}\right) \\
=\mathbb{E}\left(\mathrm{e}^{-\lambda \varepsilon^{2 N} \sigma_{b_{\varepsilon}}^{+}+i \mu \varepsilon S_{b_{\varepsilon}}^{+}} \mathbb{1}_{\left\{\sigma_{b_{\varepsilon}}^{+}<+\infty\right\}}\right) \\
=\mathrm{e}^{i \mu \varepsilon b_{\varepsilon}} \sum_{k=1}^{N} L_{k}\left(\mathrm{e}^{-\lambda \varepsilon^{2 N}}, \mathrm{e}^{i \mu \varepsilon}\right) u_{k}\left(\mathrm{e}^{-\lambda \varepsilon^{2 N}}\right)^{b_{\varepsilon}} .
\end{aligned}
$$

Recall that we previously set $u_{j}(\lambda, \varepsilon)=u_{j}\left(\mathrm{e}^{-\lambda \varepsilon^{2 N}}\right)$. Thanks to asymptotics (119), we get that

$$
\begin{gathered}
u_{k}(\lambda, \varepsilon)-u_{j}(\lambda, \varepsilon) \underset{\varepsilon \rightarrow 0^{+}}{\sim}\left(\varphi_{j}-\varphi_{k}\right) \sqrt[2 N]{\frac{\lambda}{c} \varepsilon,} \\
1-u_{j}(\lambda, \varepsilon) \mathrm{e}^{i \mu \varepsilon} \underset{\varepsilon \rightarrow 0^{+}}{\sim}\left(\varphi_{j} \sqrt[2 N]{\frac{\lambda}{c}}-i \mu\right) \varepsilon .
\end{gathered}
$$

Thus,

$$
\begin{aligned}
\lim _{\varepsilon \rightarrow 0^{+}} L_{k}\left(\mathrm{e}^{-\lambda \varepsilon^{2 N}}, \mathrm{e}^{i \mu \varepsilon}\right) & =\prod_{\substack{1 \leq j \leq N \\
j \neq k}} \frac{\varphi_{j} \sqrt[2 N]{\lambda / c}-i \mu}{\left(\varphi_{j}-\varphi_{k}\right) \sqrt[2 N]{\lambda / c}} \\
& =\prod_{\substack{1 \leq j \leq N \\
j \neq k}} \frac{\varphi_{j}}{\varphi_{j}-\varphi_{k}} \prod_{\substack{1 \leq j \leq N \\
j \neq k}}\left(1-\frac{i \overline{\varphi_{j}} \mu}{\sqrt[2 N]{\lambda / c}}\right) .
\end{aligned}
$$

Finally, we can easily conclude with the help of the elementary limits that

$$
\lim _{\varepsilon \rightarrow 0^{+}} \mathrm{e}^{i \mu \varepsilon b_{\varepsilon}}=\mathrm{e}^{i \mu b}, \quad \lim _{\varepsilon \rightarrow 0^{+}} u_{k}(\lambda, \varepsilon)^{b_{\varepsilon}}=\mathrm{e}^{-\varphi_{k} \sqrt[2 N]{\lambda / c} b} .
$$

Theorem 36. The following convergence holds:

$$
X_{b}^{\varepsilon+} \underset{\varepsilon \rightarrow 0^{+}}{\longrightarrow} X_{b}^{+} \text {, }
$$

where, for any $\mu \in \mathbb{R}$,

$$
\mathbb{E}\left(\mathrm{e}^{i \mu X_{b}^{+}} \mathbb{1}_{\left\{\tau_{b}^{+}<+\infty\right\}}\right)=\mathrm{e}^{i \mu b} \sum_{j=0}^{N-1} \frac{(-i \mu b)^{j}}{j !} .
$$


This is the Fourier transform of the pseudorandom variable $X_{b}^{+}$. Moreover,

$$
\mathbb{P}\left\{\tau_{b}^{+}<+\infty\right\}=1
$$

Proof. By (194), we have that

$$
\begin{aligned}
\mathbb{E}\left(\mathrm{e}^{i \mu X_{b}^{\varepsilon+}} \mathbb{1}_{\left\{\tau_{b}^{\varepsilon+}<+\infty\right\}}\right) & =\mathbb{E}\left(\mathrm{e}^{i \mu \varepsilon S_{b_{\varepsilon}}^{+}} \mathbb{1}_{\left\{\sigma_{b_{\varepsilon}}^{+}<+\infty\right\}}\right) \\
& =\mathrm{e}^{i \mu \varepsilon b_{\varepsilon}} \sum_{k=0}^{N-1}\left(\begin{array}{c}
j+b_{\varepsilon}-1 \\
b_{\varepsilon}-1
\end{array}\right)\left(1-\mathrm{e}^{i \mu \varepsilon}\right)^{j} .
\end{aligned}
$$

We can easily conclude by using the elementary asymptotics that

$$
\begin{gathered}
\lim _{\varepsilon \rightarrow 0^{+}} \mathrm{e}^{i \mu \varepsilon b_{\varepsilon}}=\mathrm{e}^{i \mu b}, \quad\left(\begin{array}{c}
j+b_{\varepsilon}-1 \\
b_{\varepsilon}-1
\end{array}\right) \underset{\varepsilon \rightarrow 0^{+}}{\sim} \frac{b^{j}}{j ! \varepsilon^{j}}, \\
\left(1-\mathrm{e}^{i \mu \varepsilon}\right)^{j} \underset{\varepsilon \rightarrow 0^{+}}{\sim}(-i \mu \varepsilon)^{j} .
\end{gathered}
$$

Corollary 37. The pseudodistribution of $X_{b}^{+}$is given by

$$
\frac{\mathbb{P}\left\{X_{b}^{+} \in \mathrm{d} z\right\}}{\mathrm{d} z}=\sum_{j=0}^{N-1} \frac{b^{j}}{j !} \delta_{b}^{(j)}(z) .
$$

This formula should be understood as follows: for any $(N-1)$-times differentiable function $f$, by omitting the condition $\tau_{b}^{+}<+\infty$,

$$
\mathbb{E}\left[f\left(X_{b}^{+}\right)\right]=\sum_{j=0}^{N-1}(-1)^{j} \frac{b^{j}}{j !} f^{(j)}(b) .
$$

We retrieve a result of [11] and, in the case $N=2$, a pioneering result of [18].

\section{Part III-First Exit Time from a Bounded Interval}

4.1. On the Pseudodistribution of $\left(\sigma_{a b}, S_{a b}\right)$. Let $a, b$ be two integers such that $a<0<b$ and let $\mathscr{E}=\{a, a-1, \ldots, a-$ $N+1\} \cup\{b, b+1, \ldots, b+N-1\}$. In this section, we explicitly compute the generating function of $\left(\sigma_{a b}, S_{a b}\right)$. Set, for $\ell \in \mathscr{E}$,

$$
\begin{aligned}
H_{a b, \ell}(z) & =\mathbb{E}\left(z^{\sigma_{a b}} \mathbb{1}_{\left\{S_{a b}=\ell, \sigma_{a b}<+\infty\right\}}\right) \\
& =\sum_{k \in \mathbb{N}} \mathbb{P}\left\{\sigma_{a b}=k, S_{a b}=\ell\right\} z^{k} .
\end{aligned}
$$

We are able to provide an explicit expression of $H_{a b, \ell}(z)$. As in Section 3.1, due to (62), we have the following a priori estimate: $\left|\mathbb{P}\left\{\sigma_{a b}=k, S_{a b}=\ell\right\}\right| \leq \mid \mathbb{P}\left\{S_{1} \in(a, b), \ldots, S_{k-1} \in\right.$ $\left.(a, b), S_{k}=\ell\right\} \mid \leq M_{1}^{k}$. As a byproduct, the power series defining $H_{a b, \ell}(z)$ absolutely converges for $|z|<1 / M_{1}$.

\subsubsection{Joint Pseudodistribution of $\left(\sigma_{a b}, S_{a b}\right)$}

Theorem 38. The pseudodistribution of $\left(\sigma_{a b}, S_{a b}\right)$ is characterized by the identity, valid for any $z \in(0,1)$,

$$
\mathbb{E}\left(z^{\sigma_{a b}} \mathbb{1}_{\left\{S_{a b}=\ell, \sigma_{a b}<+\infty\right\}}\right)=\frac{U_{\ell}\left(u_{1}(z), \ldots, u_{2 N}(z)\right)}{U\left(u_{1}(z), \ldots, u_{2 N}(z)\right)},
$$

where

$$
\begin{aligned}
& U\left(u_{1}, \ldots, u_{2 N}\right) \\
& =\left|\begin{array}{ccccccc}
1 & u_{1} & \cdots & u_{1}^{N-1} & u_{1}^{b-a+N-1} & \cdots & u_{1}^{b-a+2 N-2} \\
\vdots & \vdots & & \vdots & \vdots & & \vdots \\
1 & u_{2 N} & \cdots & u_{2 N}^{N-1} & u_{2 N}^{b-a+N-1} & \cdots & u_{2 N}^{b-a+2 N-2}
\end{array}\right|
\end{aligned}
$$

and, if $a-N+1 \leq \ell \leq a, U_{\ell}\left(u_{1}, \ldots, u_{2 N}\right)$ is the determinant

$$
\left|\begin{array}{ccccccccccc}
1 & u_{1} & \cdots & u_{1}^{\ell+N-a-2} & u_{1}^{N-a-1} & u_{1}^{\ell+N-a} & \cdots & u_{1}^{N-1} & u_{1}^{b-a+N-1} & \cdots & u_{1}^{b-a+2 N-2} \\
\vdots & \vdots & & \vdots & \vdots & \vdots & & \vdots & \vdots & & \vdots \\
1 & u_{2 N} & \cdots & u_{2 N}^{\ell+N-a-2} & u_{2 N}^{N-a-1} & u_{2 N}^{\ell+N-a} & \cdots & u_{2 N}^{N-1} & u_{2 N}^{b-a+N-1} & \cdots & u_{2 N}^{b-a+2 N-2}
\end{array}\right|,
$$

and if $b \leq \ell \leq b+N-1, U_{\ell}\left(u_{1}, \ldots, u_{2 N}\right)$ is the determinant

$$
\left|\begin{array}{ccccccccccc}
1 & u_{1} & \cdots & u_{1}^{N-1} & u_{1}^{b-a+N-1} & \cdots & u_{1}^{\ell+N-a-2} & u_{1}^{N-a-1} & u_{1}^{\ell+N-a} & \cdots & u_{1}^{b-a+2 N-2} \\
\vdots & \vdots & & \vdots & \vdots & & \vdots & \vdots & \vdots & & \vdots \\
1 & u_{2 N} & \cdots & u_{2 N}^{N-1} & u_{2 N}^{b-a+N-1} & \cdots & u_{2 N}^{\ell+N-a-2} & u_{2 N}^{N-a-1} & u_{2 N}^{\ell+N-a} & \cdots & u_{2 N}^{b-a+2 N-2}
\end{array}\right| .
$$


Proof. Pick an integer $k$ such that $k \leq a$ or $k \geq b$. If $S_{n}=k$, then an exit of the interval $(a, b)$ occurs before time $n: \sigma_{a b} \leq n$. This remark and the independence of the increments of the pseudorandom walk entail that

$$
\begin{aligned}
\mathbb{P}\left\{S_{n}=k\right\} & =\mathbb{P}\left\{S_{n}=k, \sigma_{a b} \leq n\right\} \\
& =\sum_{j=0}^{n} \sum_{\ell \in \mathscr{E}} \mathbb{P}\left\{S_{n}=k, \sigma_{a b}=j, S_{a b}=\ell\right\} \\
& =\sum_{j=0}^{n} \sum_{\ell \in \mathscr{E}} \mathbb{P}\left\{\sigma_{a b}=j, S_{a b}=\ell\right\} \mathbb{P}\left\{S_{n-j}=k-\ell\right\} .
\end{aligned}
$$

Thanks to the absolute convergence of the series defining $G_{k}(z)$ and $H_{a b, \ell}(z)$ for $z \in(0,1)$ and $|z|<1 / M_{1}$, respectively, we can apply the generating function to equality (235). We get, for $z \in\left(0,1 / M_{1}\right)$, that

$$
G_{k}(z)=\sum_{\ell \in \mathscr{E}} G_{k-\ell}(z) H_{a b, \ell}(z) .
$$

Using expression (94) of $G_{k}$, namely, $G_{k}(z)=$ $\sum_{j=1}^{N} \alpha_{j}(z) u_{j}(z)^{|k|}$, we get, for $k \geq b+N-1$ (recall that $\left.v_{j}(z)=1 / u_{j}(z)\right)$, that

$$
\sum_{j=1}^{N} \alpha_{j}(z) u_{j}(z)^{k}\left(\sum_{\ell \in \mathscr{E}} H_{a b, \ell}(z) v_{j}(z)^{\ell}-1\right)=0,
$$

and, for $k \leq a-N+1$, that

$$
\sum_{j=1}^{N} \alpha_{j}(z) v_{j}(z)^{k}\left(\sum_{\ell \in \mathscr{E}} H_{a b, \ell}(z) u_{j}(z)^{\ell}-1\right)=0 .
$$

When limiting the range of $k$ to the set $\{b+N, b+N+1, \ldots, b+$ $2 N-1\}$ in (237) and to the set $\{a-2 N+1, a-2 N+2, \ldots, a-$ $N\}$ in (238), we see that (237) and (238) are homogeneous Vandermonde systems whose solutions are trivial; that is, the terms within parentheses in (237) and (238) vanish. Thus, we get the two systems below:

$$
\begin{aligned}
& \sum_{\ell \in \mathscr{E}} H_{a b, \ell}(z) u_{j}(z)^{\ell}=1, \quad 1 \leq j \leq N, \\
& \sum_{\ell \in \mathscr{E}} H_{a b, \ell}(z) v_{j}(z)^{\ell}=1, \quad 1 \leq j \leq N .
\end{aligned}
$$

It will be convenient to relabel the $u_{j}(z)$ 's and $v_{j}(z)$ 's, $1 \leq j \leq$ $N$, as $u_{j}(z)=v_{j+N}(z)$ and $v_{j}(z)=u_{j+N}(z)$; note that $v_{j}(z)=$ $1 / u_{j}(z)$ for any $j \in\{1, \ldots, 2 N\}$ and $\left\{u_{1}(z), \ldots, u_{2 N}(z)\right\}=$ $\left\{v_{1}(z), \ldots, v_{2 N}(z)\right\}$. By using the relabeling $u_{j}(z), v_{j}(z), 1 \leq$ $j \leq 2 \mathrm{~N}$, we obtain the two equivalent following systems of $2 \mathrm{~N}$ equations and $2 N$ unknowns, $u_{1}(z), \ldots, u_{2 N}(z)$ for the first one, $v_{1}(z), \ldots, v_{2 N}(z)$ for the second one:

$$
\begin{aligned}
& \sum_{\ell \in \mathscr{E}} H_{a b, \ell}(z) u_{j}(z)^{\ell}=1, \quad 1 \leq j \leq 2 N \\
& \sum_{\ell \in \mathscr{E}} H_{a b, \ell}(z) v_{j}(z)^{\ell}=1, \quad 1 \leq j \leq 2 N .
\end{aligned}
$$

Systems (240) and (241) are "lacunary" Vandermonde systems (some powers of $u_{j}(z)$ are missing). For instance, let us rewrite system (240) as

$$
\sum_{\ell \in \mathscr{E}} H_{a b, \ell}(z) u_{j}(z)^{\ell+N-a-1}=u_{j}(z)^{N-a-1}, \quad 1 \leq j \leq 2 N .
$$

Cramer's formulae immediately yield (231) at least for $z \in$ $\left(0,1 / M_{1}\right)$. By analyticity of the $u_{j}$ 's on $(0,1)$, it is easily seen that (231) holds true for $z \in(0,1)$. Systems (240) and (241) will be used in Lemma 42 .

A method for computing the determinants exhibited in Theorem 38 and solving system (242) is proposed in Appendix A.1. In particular, we can deduce from Proposition A.3 an alternative representation of $\mathbb{E}\left(z^{\sigma_{a b}} \mathbb{1}_{\left\{S_{a b}=\ell, \sigma_{a b}<+\infty\right\}}\right)$ which can be seen as the analogous of (124). Set $s_{0}(z)=1$ and, for $k, \ell \in\{1, \ldots, 2 N\}$,

$$
\begin{gathered}
s_{\ell}(z)=\sum_{1 \leq i_{1}<\cdots<i_{\ell} \leq 2 N} u_{i_{1}}(z) \cdots u_{i_{\ell}}(z), \\
p_{k}(z)=\prod_{\substack{1 \leq i \leq 2 N \\
i \neq k}}\left[u_{k}(z)-u_{i}(z)\right],
\end{gathered}
$$

$s_{k, 0}(z)=1, s_{k, m}(z)=0$ for any integer $m$ such that $m \leq-1$ or $m \geq 2 N$ and, for $m \in\{1, \ldots, 2 N-1\}$,

$$
s_{k, m}(z)=\sum_{\substack{1 \leq i_{1}<\cdots<i_{m} \leq 2 N \\ i_{1}, \ldots, i_{m} \neq k}} u_{i_{1}}(z) \cdots u_{i_{m}}(z) .
$$

Set also

$$
\begin{gathered}
\widetilde{U}(z)=\left|\begin{array}{cccc}
s_{N}(z) & s_{N-1}(z) & \cdots & s_{N-b+a+2}(z) \\
s_{N+1}(z) & s_{N}(z) & \cdots & s_{N-b+a+1}(z) \\
\vdots & \vdots & & \vdots \\
s_{N+b-a-2}(z) & s_{N+b-a-3}(z) & \cdots & s_{N}(z)
\end{array}\right|, \\
\widetilde{U}_{k \ell}(z)=\left|\begin{array}{cccc}
s_{k, N}(z) & s_{k, N-1}(z) & \cdots & s_{k, N-b+a+1}(z) \\
s_{k, N+1}(z) & s_{k, N}(z) & \cdots & s_{k, N-b+a}(z) \\
\vdots & \vdots & & \vdots \\
s_{k, N+b-a-2}(z) & s_{k, N+b-a-3}(z) & \cdots & s_{k, N-1}(z) \\
s_{k, N+b-\ell-1}(z) & s_{k, N+b-\ell-2}(z) & \cdots & s_{k, N+a-\ell}(z)
\end{array}\right| .
\end{gathered}
$$

Then, applying Proposition A.3 with the choices $p=r=N$ and $q=b-a-1$ leads, for any $\ell \in \mathscr{E}$, to

$$
\mathbb{E}\left(z^{\sigma_{a b}} \mathbb{1}_{\left\{S_{a b}=\ell, \sigma_{a b}<+\infty\right\}}\right)=\frac{(-1)^{\ell+N-a-1}}{\widetilde{U}(z)} \sum_{k=1}^{2 N} \frac{\widetilde{U}_{k \ell}(z)}{p_{k}(z)} u_{k}(z)^{N-a-1} .
$$

The double generating function defined by

$$
\mathbb{E}\left(z^{\sigma_{a b}} \zeta^{S_{a b}} \mathbb{1}_{\left\{\sigma_{a b}<+\infty\right\}}\right)=\sum_{\ell \in \mathscr{E}} \mathbb{E}\left(z^{\sigma_{a b}} \mathbb{1}_{\left\{S_{a b}=\ell, \sigma_{a b}<+\infty\right\}}\right) \zeta^{\ell}
$$

admits an interesting representation by means of interpolating polynomials that we display in the following theorem. 
Theorem 39. The double generating function of $\left(\sigma_{a b}, S_{a b}\right)$ is given, for $z \in\left(0,1 / M_{1}\right)$ and $\zeta \in \mathbb{C}$, by

$$
\mathbb{E}\left(z^{\sigma_{a b}} \zeta^{S_{a b}} \mathbb{1}_{\left\{\sigma_{a b}<+\infty\right\}}\right)=\sum_{k=1}^{2 N} \widetilde{L}_{k}(z, \zeta)\left(v_{k}(z) \zeta\right)^{a-N+1},
$$

where, for $k \in\{1, \ldots, 2 N\}$

$$
\widetilde{L}_{k}(z, \zeta)=\frac{U\left(u_{1}(z), \ldots, u_{k-1}(z), \zeta, u_{k+1}(z), \ldots, u_{2 N}(z)\right)}{U\left(u_{1}(z), \ldots, u_{2 N}(z)\right)}
$$

The functions $\zeta \mapsto \widetilde{L}_{k}(z, \zeta)$ are interpolating polynomials satisfying $\widetilde{L}_{k}\left(z, u_{j}(z)\right)=\delta_{j k}$ and can be expressed as

$$
\widetilde{L}_{k}(z, \zeta)=P_{k}(z, \zeta) \prod_{\substack{1 \leq j \leq 2 N \\ j \neq k}} \frac{\zeta-u_{j}(z)}{u_{k}(z)-u_{j}(z)}
$$

where $\zeta \mapsto P_{k}(z, \zeta)$ are some polynomials of degree $(b-a-1)$.
Proof. By (231), we have that

$$
\begin{aligned}
& \mathbb{E}\left(z^{\sigma_{a b}} \zeta^{S_{a b}} \mathbb{1}_{\left\{S_{a b} \leq a, \sigma_{a b}<+\infty\right\}}\right) \\
& =\sum_{\ell=a-N+1}^{a} H_{a b, \ell}(z) \zeta^{\ell}=\sum_{\ell=a-N+1}^{a} \frac{U_{\ell}\left(u_{1}(z), \ldots, u_{2 N}(z)\right)}{U\left(u_{1}(z), \ldots, u_{2 N}(z)\right)} \zeta^{\ell} .
\end{aligned}
$$

In order to simplify the text, we omit the variable $z$. We expand the determinant $U_{\ell}\left(u_{1}, \ldots, u_{2 N}\right), a-N+1 \leq \ell \leq a$ with respect to its $(\ell+N-a)$ th column:

$$
\begin{aligned}
& U_{\ell}\left(u_{1}, \ldots, u_{2 N}\right) \\
& \quad=\sum_{k=1}^{2 N} u_{k}^{N-a-1} U_{k \ell}\left(u_{1}, \ldots, u_{k-1}, u_{k+1}, \ldots, u_{2 N}\right),
\end{aligned}
$$

where $U_{k \ell}\left(u_{1}, \ldots, u_{k-1}, u_{k+1}, \ldots, u_{2 N}\right), 1 \leq k \leq 2 N$, is the determinant

$$
\left|\begin{array}{ccccccccccc}
1 & u_{1} & \cdots & u_{1}^{\ell+N-a-2} & 0 & u_{1}^{\ell+N-a} & \cdots & u_{1}^{N-1} & u_{1}^{b-a+N-1} & \cdots & u_{1}^{b-a+2 N-2} \\
\vdots & \vdots & & \vdots & \vdots & \vdots & & \vdots & \vdots & & \vdots \\
1 & u_{k-1} & \cdots & u_{k-1}^{\ell+N-a-2} & 0 & u_{k-1}^{\ell+N-a} & \cdots & u_{k-1}^{N-1} & u_{k-1}^{b-a+N-1} & \cdots & u_{k-1}^{b-a+2 N-2} \\
1 & u_{k} & \cdots & u_{k}^{\ell+N-a-2} & 1 & u_{k}^{\ell+N-a} & \cdots & u_{k}^{N-1} & u_{k}^{b-a+N-1} & \cdots & u_{k}^{b-a+2 N-2} \\
1 & u_{k+1} & \cdots & u_{k+1}^{\ell+N-a-2} & 0 & u_{k+1}^{\ell+N-a} & \cdots & u_{k+1}^{N-1} & u_{k+1}^{b-a+N-1} & \cdots & u_{k+1}^{b-a+2 N-2} \\
\vdots & \vdots & & \vdots & \vdots & \vdots & & \vdots & \vdots & & \vdots \\
1 & u_{2 N} & \cdots & u_{2 N}^{\ell+N-a-2} & 0 & u_{2 N}^{\ell+N-a} & \cdots & u_{2 N}^{N-1} & u_{2 N}^{b-a+N-1} & \cdots & u_{2 N}^{b-a+2 N-2}
\end{array}\right|
$$

which plainly coincides with

$$
\left|\begin{array}{ccccccccccc}
1 & u_{1} & \cdots & u_{1}^{\ell+N-a-2} & u_{1}^{\ell+N-a-1} & u_{1}^{\ell+N-a} & \cdots & u_{1}^{N-1} & u_{1}^{b-a+N-1} & \cdots & u_{1}^{b-a+2 N-2} \\
\vdots & \vdots & & \vdots & \vdots & \vdots & & \vdots & \vdots & & \vdots \\
1 & u_{k-1} & \cdots & u_{k-1}^{\ell+N-a-2} & u_{k-1}^{\ell+N-a-1} & u_{k-1}^{\ell+N-a} & \cdots & u_{k-1}^{N-1} & u_{k-1}^{b-a+N-1} & \cdots & u_{k-1}^{b-a+2 N-2} \\
0 & 0 & \cdots & 0 & 1 & 0 & \cdots & 0 & 0 & \cdots & 0 \\
1 & u_{k+1} & \cdots & u_{k+1}^{\ell+N-a-2} & u_{k+1}^{\ell+N-a-1} & u_{k+1}^{\ell+N-a} & \cdots & u_{k+1}^{N-1} & u_{k+1}^{b-a+N-1} & \cdots & u_{k+1}^{b-a+2 N-2} \\
\vdots & \vdots & & \vdots & \vdots & \vdots & & \vdots & \vdots & & \vdots \\
1 & u_{2 N} & \cdots & u_{2 N}^{\ell+N-a-2} & u_{2 N}^{\ell+N-a-1} & u_{2 N}^{\ell+N-a} & \cdots & u_{2 N}^{N-1} & u_{2 N}^{b-a+N-1} & \cdots & u_{2 N}^{b-a+2 N-2}
\end{array}\right| .
$$

Therefore, we obtain that

$$
\begin{aligned}
& \sum_{\ell=a-N+1}^{a} U_{\ell}\left(u_{1}, \ldots, u_{2 N}\right) \zeta^{\ell} \\
= & \sum_{\ell=a-N+1}^{a}\left(\sum_{k=1}^{2 N} u_{k}^{N-a-1} U_{k \ell}\left(u_{1}, \ldots, u_{k-1}, u_{k+1}, \ldots, u_{2 N}\right)\right) \zeta^{\ell}
\end{aligned}
$$$$
=\zeta^{a-N+1}
$$$$
\times \sum_{k=1}^{2 N}\left(\sum_{\ell=a-N+1}^{a} U_{k \ell}\left(u_{1}, \ldots, u_{k-1}, u_{k+1}, \ldots, u_{2 N}\right) \zeta^{\ell+N-a-1}\right)
$$$$
\times v_{k}^{a-N+1}
$$ 
Next, we can see that the foregoing sum within parentheses is the expansion of the determinant $D_{k}^{-}(z, \zeta)$ below with respect to its $k$ th row (by putting back the variable $z$ ):

$$
\left|\begin{array}{cccccccc}
1 & u_{1}(z) & \cdots & u_{1}^{N-1}(z) & u_{1}^{b-a+N-1}(z) & \cdots & u_{1}^{b-a+2 N-2}(z) \\
\vdots & \vdots & & \vdots & \vdots & & \vdots \\
1 & u_{k-1}(z) & \cdots & u_{k-1}^{N-1}(z) & u_{k-1}^{b-a+N-1}(z) & \cdots & u_{k-1}^{b-a+2 N-2}(z) \\
1 & \zeta & \cdots & \zeta^{N-1} & 0 & \cdots & 0 \\
1 & u_{k+1}(z) & \cdots & u_{k+1}^{N-1}(z) & u_{k+1}^{b-a+N-1}(z) & \cdots & u_{k+1}^{b-a+2 N-2}(z) \\
\vdots & \vdots & & \vdots & \vdots & & \vdots \\
1 & u_{2 N}(z) & \cdots & u_{2 N}^{N-1}(z) & u_{2 N}^{b-a+N-1}(z) & \cdots & u_{2 N}^{b-a+2 N-2}(z)
\end{array}\right| .
$$

As a result, by setting

$D(z)$

$=U\left(u_{1}(z), \ldots, u_{2 N}(z)\right)$

$=\left|\begin{array}{ccccccc}1 & u_{1}(z) & \cdots & u_{1}^{N-1}(z) & u_{1}^{b-a+N-1}(z) & \cdots & u_{1}^{b-a+2 N-2}(z) \\ \vdots & \vdots & & \vdots & \vdots & & \vdots \\ 1 & u_{2 N}(z) & \cdots & u_{2 N}^{N-1}(z) & u_{2 N}^{b-a+N-1}(z) & \cdots & u_{2 N}^{b-a+2 N-2}(z)\end{array}\right|$,

we obtain that

$\mathbb{E}\left(z^{\sigma_{a b}} \zeta^{S_{a b}} \mathbb{1}_{\left\{S_{a b} \leq a, \sigma_{a b}<+\infty\right\}}\right)=\frac{1}{D(z)} \sum_{k=1}^{N} D_{k}^{-}(z, \zeta)\left(v_{k}(z) \zeta\right)^{a-N+1}$.

Similarly, we could check that

$\mathbb{E}\left(z^{\sigma_{a b}} \zeta^{S_{a b}} \mathbb{1}_{\left\{S_{a b} \geq b, \sigma_{a b}<+\infty\right\}}\right)=\frac{1}{D(z)} \sum_{k=1}^{N} D_{k}^{+}(z, \zeta)\left(v_{k}(z) \zeta\right)^{a-N+1}$,

where $D_{k}^{+}(z, \zeta)$ is the determinant

$$
\left|\begin{array}{ccccccc}
1 & u_{1}(z) & \cdots & u_{1}^{N-1}(z) & u_{1}^{b-a+N-1}(z) & \cdots & u_{1}^{b-a+2 N-2}(z) \\
\vdots & \vdots & & \vdots & \vdots & & \vdots \\
1 & u_{k-1}(z) & \cdots & u_{k-1}^{N-1}(z) & u_{k-1}^{b-a+N-1}(z) & \cdots & u_{k-1}^{b-a+2 N-2}(z) \\
0 & 0 & \cdots & 0 & \zeta^{b-a+N-1} & \cdots & \zeta^{b-a+2 N-2} \\
1 & u_{k+1}(z) & \cdots & u_{k+1}^{N-1}(z) & u_{k+1}^{b-a+N-1}(z) & \cdots & u_{k+1}^{b-a+2 N-2}(z) \\
\vdots & \vdots & & \vdots & \vdots & & \vdots \\
1 & u_{2 N}(z) & \cdots & u_{2 N}^{N-1}(z) & u_{2 N}^{b-a+N-1}(z) & \cdots & u_{2 N}^{b-a+2 N-2}(z)
\end{array}\right| .
$$

By adding (258) and (259) and setting

$$
\begin{aligned}
D_{k}(z, \zeta) & =D_{k}^{+}(z, \zeta)+D_{k}^{-}(z, \zeta) \\
& =U\left(u_{1}(z), \ldots, u_{k-1}(z), \zeta, u_{k+1}(z), \ldots, u_{2 N}(z)\right)
\end{aligned}
$$

we obtain that

$$
\mathbb{E}\left(z^{\sigma_{a b}} \zeta^{S_{a b}} \mathbb{1}_{\left\{\sigma_{a b}<+\infty\right\}}\right)=\frac{1}{D(z)} \sum_{k=1}^{2 N} D_{k}(z, \zeta)\left(v_{k}(z) \zeta\right)^{a-N+1} .
$$

We observe that the polynomials $\widetilde{L}_{k}(z, \zeta)=D_{k}(z, \zeta) / D(z)$ with respect to the variable $\zeta$ are of degree $b-a+2 N-2$ and satisfy the equalities $\widetilde{L}_{k}\left(z, u_{j}(z)\right)=\delta_{j k}$ for all $j \in \mathscr{E}$ and $k \in\{1, \ldots, 2 N\}$. Hence they can be expressed by means of the Lagrange fundamental polynomials as displayed in Theorem 39.

Example 40. For $N=1$, (248) reads

$$
\begin{aligned}
\mathbb{E}\left(z^{\sigma_{a b}} \zeta^{S_{a b}} \mathbb{1}_{\left\{\sigma_{a b}<+\infty\right\}}\right)= & \widetilde{L}_{1}(z, \zeta)\left(v_{1}(z) \zeta\right)^{a} \\
& +\widetilde{L}_{2}(z, \zeta)\left(v_{2}(z) \zeta\right)^{a},
\end{aligned}
$$

where

$$
\begin{aligned}
& \widetilde{L}_{1}(z, \zeta)=\frac{U\left(\zeta, u_{2}(z)\right)}{U\left(u_{1}(z), u_{2}(z)\right)}=\frac{u_{2}(z)^{b-a}-\zeta^{b-a}}{u_{2}(z)^{b-a}-u_{1}(z)^{b-a}} \\
& \widetilde{L}_{2}(z, \zeta)=\frac{U\left(u_{1}(z), \zeta\right)}{U\left(u_{1}(z), u_{2}(z)\right)}=\frac{\zeta^{b-a}-u_{1}(z)^{b-a}}{u_{2}(z)^{b-a}-u_{1}(z)^{b-a}}
\end{aligned}
$$

Since $v_{1}(z)=1 / u_{1}(z)$ and $v_{2}(z)=1 / u_{2}(z)$, and also $u_{2}(z)=$ $1 / u_{1}(z)$

$$
\begin{aligned}
\mathbb{E}\left(z^{\sigma_{a b}} \zeta^{S_{a b}} \mathbb{1}_{\left\{\sigma_{a b}<+\infty\right\}}\right)= & \frac{u_{2}(z)^{b}-u_{1}(z)^{b}}{u_{2}(z)^{b-a}-u_{1}(z)^{b-a}} \zeta^{a} \\
& +\frac{u_{2}(z)^{a}-u_{1}(z)^{a}}{u_{2}(z)^{a-b}-u_{1}(z)^{a-b}} \zeta^{b}
\end{aligned}
$$

from which we extract the well-known formulae related to the case of an ordinary random walk:

$$
\begin{aligned}
& \mathbb{E}\left(z^{\sigma_{a b}} \mathbb{1}_{\left\{S_{a b}=a, \sigma_{a b}<+\infty\right\}}\right)=\frac{u_{2}(z)^{b}-u_{1}(z)^{b}}{u_{2}(z)^{b-a}-u_{1}(z)^{b-a}}, \\
& \mathbb{E}\left(z^{\sigma_{a b}} \mathbb{1}_{\left\{S_{a b}=b, \sigma_{a b}<+\infty\right\}}\right)=\frac{u_{2}(z)^{a}-u_{1}(z)^{a}}{u_{2}(z)^{a-b}-u_{1}(z)^{a-b}} .
\end{aligned}
$$

In particular, if $c=1 / 2$ (case of the classical random walk), by Example 10, we have in the above formulae that

$$
u_{1}(z)=\frac{1-\sqrt{1-z^{2}}}{z}, \quad u_{2}(z)=\frac{1+\sqrt{1-z^{2}}}{z} .
$$

For $N=2,(248)$ reads

$$
\begin{aligned}
\mathbb{E}\left(z^{\sigma_{a b}} \zeta^{S_{a b}} \mathbb{1}_{\left\{\sigma_{a b}<+\infty\right\}}\right) & \\
= & \widetilde{L}_{1}(z, \zeta)\left(v_{1}(z) \zeta\right)^{a-1}+\widetilde{L}_{2}(z, \zeta)\left(v_{2}(z) \zeta\right)^{a-1} \\
& +\widetilde{L}_{3}(z, \zeta)\left(v_{3}(z) \zeta\right)^{a-1}+\widetilde{L}_{4}(z, \zeta)\left(v_{4}(z) \zeta\right)^{a-1}
\end{aligned}
$$


where

$$
\begin{aligned}
\widetilde{L}_{1}(z, \zeta) & =\frac{U\left(\zeta, u_{2}(z), u_{3}(z), u_{4}(z)\right)}{U\left(u_{1}(z), u_{2}(z), u_{3}(z), u_{4}(z)\right)}, \\
\widetilde{L}_{2}(z, \zeta) & =\frac{U\left(u_{1}(z), \zeta, u_{3}(z), u_{4}(z)\right)}{U\left(u_{1}(z), u_{2}(z), u_{3}(z), u_{4}(z)\right)}, \\
\widetilde{L}_{3}(z, \zeta) & =\frac{U\left(u_{1}(z), u_{2}(z), \zeta, u_{4}(z)\right)}{U\left(u_{1}(z), u_{2}(z), u_{3}(z), u_{4}(z)\right)}, \\
\widetilde{L}_{4}(z, \zeta) & =\frac{U\left(u_{1}(z), u_{2}(z), u_{3}(z), \zeta\right)}{U\left(u_{1}(z), u_{2}(z), u_{3}(z), u_{4}(z)\right)} .
\end{aligned}
$$

All the polynomials $\widetilde{L}_{k}(z, \zeta), 1 \leq k \leq 4$, have the form $A_{k, a-1}(z)+A_{k, a}(z) \zeta+A_{k, b}(z) \zeta^{b-a+1}+A_{k, b+1}(z) \zeta^{b-a+2}$.

Remark 41. By expanding the determinant $D_{k}(z, \zeta)$ with respect to its $k$ th raw, we obtain an expansion for the polynomial $\widetilde{L}(z, \zeta)$ as a linear combination of $1, \zeta, \ldots, \zeta^{N-1}$, $\zeta^{b-a+N-1}, \zeta^{b-a+N}, \ldots, \zeta^{b-a+2 N-2}$, that is, an expansion of the form

$$
\begin{aligned}
\tilde{L}_{k}(z, \zeta) & =\sum_{\ell=0}^{N-1} A_{k, \ell+a-N+1}(z) \zeta^{\ell}+\sum_{\ell=b-a+N-1}^{b-a+2 N-2} A_{k, \ell+a-N+1}(z) \zeta^{\ell} \\
& =\zeta^{N-a-1} \sum_{\ell \in \mathscr{E}} A_{k \ell}(z) \zeta^{\ell} .
\end{aligned}
$$

Hence,

$$
\begin{aligned}
\mathbb{E}\left(z^{\sigma_{a b}} \zeta^{S_{a b}} \mathbb{1}_{\left\{\sigma_{a b}<+\infty\right\}}\right) & =\sum_{k=1}^{2 N}\left(\sum_{\ell \in \mathscr{E}} A_{k \ell}(z) \zeta^{\ell}\right) v_{k}(z)^{a-N+1} \\
& =\sum_{\ell \in \mathscr{E}}\left(\sum_{k=1}^{2 N} A_{k \ell}(z) v_{k}(z)^{a-N+1}\right) \zeta^{\ell}
\end{aligned}
$$

from which we extract, for any $\ell \in \mathscr{E}$, that

$$
\mathbb{E}\left(z^{\sigma_{a b}} \mathbb{1}_{\left\{S_{a b}=\ell, \sigma_{a b}<+\infty\right\}}\right)=\sum_{k=1}^{2 N} A_{k \ell}(z) u_{k}(z)^{N-a-1} .
$$

Actually, the foregoing sum comes from the quotient $U_{\ell}\left(u_{1}(z), \ldots, u_{2 N}(z)\right) / U\left(u_{1}(z), \ldots, u_{2 N}(z)\right)$ given by (231) by expanding the determinant $U_{\ell}\left(u_{1}(z), \ldots, u_{2 N}(z)\right)$ with respect to the $(\ell+N-a)$ th column or $(\ell+N-b+1)$ th column according as the number $\ell$ satisfies $a-N+1 \leq \ell \leq a$ or $b \leq \ell \leq b+N-1$.

4.1.2. Pseudodistribution of $S_{a b}$. In order to derive the pseudodistribution of $S_{a b}$ which is characterized by the numbers $H_{a b, \ell}(1), \ell \in \mathscr{E}$, we solve the systems obtained by taking the limit in (262) as $z \rightarrow 1^{-}$.
Lemma 42. The following identities hold: for $N \leq k \leq 2 N-1$,

$$
\begin{gathered}
\sum_{\ell=b}^{b+N-1}\left(\begin{array}{c}
\ell+N-a-1 \\
k
\end{array}\right) H_{a b, \ell}(1)=\left(\begin{array}{c}
N-a-1 \\
k
\end{array}\right), \\
\sum_{\ell=a-N+1}^{a}\left(\begin{array}{c}
b+N-1-\ell \\
k
\end{array}\right) H_{a b, \ell}(1)=\left(\begin{array}{c}
b+N-1 \\
k
\end{array}\right) .
\end{gathered}
$$

Proof. By (85), we have the expansion $u_{j}(z)=1+\varepsilon_{j}(z)$, where $\varepsilon_{j}(z) \underset{z \rightarrow 1^{-}}{=} \mathcal{O}(\sqrt[2 N]{1-z})$ for any $j \in\{1, \ldots, N\}$. Actually such asymptotics holds true for any $j \in\{1, \ldots, 2 N\}$ because of the equality $u_{j}=1 / u_{j-N}$ for $j \in\{N+1, \ldots, 2 N\}$. We put this into systems (240) and (241). For doing this, it is convenient to rewrite the latter as

$$
\begin{array}{ll}
\sum_{\ell \in \mathscr{E}} H_{a b, \ell}(z) u_{j}(z)^{\ell+N-a-1}=u_{j}(z)^{N-a-1}, & 1 \leq j \leq 2 N, \\
\sum_{\ell \in \mathscr{E}} H_{a b, \ell}(z) u_{j}(z)^{b+N-1-\ell}=u_{j}(z)^{b+N-1}, & 1 \leq j \leq 2 N .
\end{array}
$$

We obtain that

$$
\begin{aligned}
\sum_{\ell \in \mathscr{E}}(1 & \left.+\varepsilon_{j}(z)\right)^{\ell+N-a-1} H_{a b, \ell}(z) \\
& =\left(1+\varepsilon_{j}(z)\right)^{N-a-1}, \quad 1 \leq j \leq 2 N, \\
\sum_{\ell \in \mathscr{E}}(1 & \left.+\varepsilon_{j}(z)\right)^{b+N-1-\ell} H_{a b, \ell}(z) \\
= & \left(1+\varepsilon_{j}(z)\right)^{b+N-1}, \quad 1 \leq j \leq 2 N .
\end{aligned}
$$

System (276) writes

$$
\begin{gathered}
\sum_{k=0}^{b-a+2 N-2}\left(\sum_{\substack{\ell \in \mathscr{E}: \\
\ell \geq k+a-N+1}}\left(\begin{array}{c}
\ell+N-a-1 \\
k
\end{array}\right) H_{a b, \ell}(z)\right) \varepsilon_{j}(z)^{k} \\
=\sum_{k=0}^{N-a-1}\left(\begin{array}{c}
N-a-1 \\
k
\end{array}\right) \varepsilon_{j}(z)^{k} .
\end{gathered}
$$

Set

$$
\begin{gathered}
M_{k}(z)=\sum_{\substack{\ell \in \mathscr{E}: \\
\ell \geq k+a-N+1}}\left(\begin{array}{c}
\ell+N-a-1 \\
k
\end{array}\right) H_{a b, \ell}(z)-\left(\begin{array}{c}
N-a-1 \\
k
\end{array}\right), \\
R_{j}(z)=-\sum_{k=2 N}^{b-a+2 N-2} M_{k}(z) \varepsilon_{j}(z)^{k} .
\end{gathered}
$$

Then, equality (278) reads

$$
\sum_{k=0}^{2 N-1} M_{k}(z) \varepsilon_{j}(z)^{k}=R_{j}(z), \quad 1 \leq j \leq 2 N .
$$


This is a Vandermonde system which can be solved as in the proof of Lemma 21 upon changing $N$ into $2 N$. We can check that $\lim _{z \rightarrow 1^{-}} M_{k}(z)=0$, which entails that

$$
\begin{aligned}
& \sum_{\substack{\ell \in \mathscr{E}: \\
\ell \geq k+a-N+1}}\left(\begin{array}{c}
\ell+N-a-1 \\
k
\end{array}\right) H_{a b, \ell}(1) \\
& \quad=\left(\begin{array}{c}
N-a-1 \\
k
\end{array}\right), \quad 0 \leq k \leq 2 N-1 .
\end{aligned}
$$

Similarly, using (277), we can prove that

$$
\begin{aligned}
& \sum_{\substack{\ell \in \mathscr{E}: \\
\ell \leq b+N-1-k}}\left(\begin{array}{c}
b+N-\ell-1 \\
k
\end{array}\right) H_{a b, \ell}(1) \\
& =\left(\begin{array}{c}
N+b-1 \\
k
\end{array}\right), \quad 0 \leq j \leq 2 N-1 .
\end{aligned}
$$

Actually, we will only use (281) and (282) restricted to $j \in$ $\{N, \ldots, 2 N-1\}$ which immediately yields system (273) and (274).

Now, we state one of the most important result of this work. We solve the famous problem of the "gambler's ruin" in the context of the pseudorandom walk.

Theorem 43. The pseudodistribution of $S_{a b}$ is given, for $\ell \in$ $\{0,1, \ldots, N-1\}$, by

$$
\begin{aligned}
& \mathbb{P}\left\{S_{a b}=a-\ell, \sigma_{a b}<+\infty\right\}=\frac{(-1)^{\ell}(K N /(\ell-a))\left(\begin{array}{c}
N-1 \\
\ell
\end{array}\right)}{\left(\begin{array}{c}
\ell+b-a+N-1 \\
N
\end{array}\right)}, \\
& \mathbb{P}\left\{S_{a b}=b+\ell, \sigma_{a b}<+\infty\right\}=\frac{(-1)^{\ell}(K N /(\ell+b))\left(\begin{array}{c}
N-1 \\
\ell
\end{array}\right)}{\left(\begin{array}{c}
\ell+b-a+N-1 \\
N
\end{array}\right)},
\end{aligned}
$$

where

$$
\begin{aligned}
K & =\left(\begin{array}{c}
N-a-1 \\
N
\end{array}\right)\left(\begin{array}{c}
N+b-1 \\
N
\end{array}\right) \\
& =\frac{(-1)^{N}}{N !} a(a-1) \cdots(a-N+1) b(b+1) \cdots(b+N-1) .
\end{aligned}
$$

$$
\begin{aligned}
& \text { Moreover, } \mathbb{P}\left\{\sigma_{a b}<+\infty\right\}=1 \text { and } \\
& \begin{aligned}
\mathbb{P}\left\{\sigma_{b}^{+}<\sigma_{a}^{-}\right\} \\
\quad=K N^{2} \iint_{D^{+}} u^{-a-1}(1-u)^{N-1} v^{b-1}(1-v)^{N-1} \mathrm{~d} u \mathrm{~d} v, \\
\mathbb{P}\left\{\sigma_{a}^{-}<\sigma_{b}^{+}\right\} \\
\quad=K N^{2} \iint_{D^{-}} u^{-a-1}(1-u)^{N-1} v^{b-1}(1-v)^{N-1} \mathrm{~d} u \mathrm{~d} v,
\end{aligned}
\end{aligned}
$$

where

$$
\begin{aligned}
& \mathscr{D}^{+}=\left\{(u, v) \in \mathbb{R}^{2}: 0 \leq v \leq u \leq 1\right\}, \\
& \mathscr{D}^{-}=\left\{(u, v) \in \mathbb{R}^{2}: 0 \leq u \leq v \leq 1\right\} .
\end{aligned}
$$

Proof. We have to solve system (273) and (274). For (273), for instance, the principal matrix and the right-hand side matrix are

$$
\left[\left(\begin{array}{c}
\ell+b-a+N-1 \\
k+N
\end{array}\right)\right]_{0 \leq k, \ell \leq N-1}, \quad\left[\left(\begin{array}{c}
N-a-1 \\
k+N
\end{array}\right)\right]_{0 \leq k \leq N-1}
$$

and the matrix form of the solution is given by

$$
\left[\left(\begin{array}{c}
\ell+b-a+N-1 \\
k+N
\end{array}\right)\right]_{0 \leq k, \ell \leq N-1}^{-1}\left[\left(\begin{array}{c}
N-a-1 \\
k+N
\end{array}\right)\right]_{0 \leq k \leq N-1} .
$$

The computation of this product being quite fastidious, we postponed it to Appendix A.3. The result is given by Theorem A.8:

$$
\left[\frac{(-1)^{\ell}(K N /(\ell+b))\left(\begin{array}{c}
N-1 \\
\ell
\end{array}\right)}{\left(\begin{array}{c}
\ell+b-a+N-1 \\
N
\end{array}\right)}\right]_{0 \leq \ell \leq N-1} .
$$

The entries of this matrix provide the pseudo-probabilities $\mathbb{P}\left\{S_{a b}=b+\ell, \sigma_{a b}<+\infty\right\}, 0 \leq \ell \leq N-1$, which are exhibited in Theorem 43. The analogous formula for $\mathbb{P}\left\{S_{a b}=\right.$ $\left.a-\ell, \sigma_{a b}<+\infty\right\}$ holds true in the same way.

Next, by observing that $\sigma_{a b}=\min \left(\sigma_{a}^{-}, \sigma_{b}^{+}\right)$and that $\left\{\sigma_{a b}<\right.$ $+\infty\}=\left\{\sigma_{a}^{-}<+\infty\right\} \cup\left\{\sigma_{b}^{+}<+\infty\right\}$, we have that

$$
\begin{aligned}
\mathbb{P}\left\{\sigma_{b}^{+}\right. & \left.<\sigma_{a}^{-}\right\} \\
& =\mathbb{P}\left\{\sigma_{b}^{+}<\sigma_{a}^{-}, \sigma_{a b}<+\infty\right\}=\mathbb{P}\left\{S_{a b} \geq b, \sigma_{a b}<+\infty\right\}
\end{aligned}
$$

$$
\begin{aligned}
& =\sum_{\ell=0}^{N-1} \mathbb{P}\left\{S_{a b}=b+\ell, \sigma_{a b}<+\infty\right\} \\
& =K \sum_{\ell=0}^{N-1} \frac{(-1)^{\ell}(N /(\ell+b))\left(\begin{array}{c}
N-1 \\
\ell
\end{array}\right)}{\left(\begin{array}{c}
\ell+b-a+N-1 \\
N
\end{array}\right)} .
\end{aligned}
$$

Noticing that $1 /(\ell+b)=\int_{0}^{1} y^{\ell+b-1} \mathrm{~d} y$ and $1 /\left(\begin{array}{c}\ell+b-a+N-1 \\ N\end{array}\right)=$ $N \int_{0}^{1} x^{\ell+b-a-1}(1-x)^{N-1} \mathrm{~d} x$, we get that

$$
\begin{gathered}
\sum_{\ell=0}^{N-1} \frac{(-1)^{\ell}(N /(\ell+b))\left(\begin{array}{c}
N-1 \\
\ell
\end{array}\right)}{\left(\begin{array}{c}
\ell+b-a+N-1 \\
N
\end{array}\right)} \\
=N^{2} \int_{0}^{1} \int_{0}^{1}\left(\begin{array}{c}
N-1 \\
\ell=0
\end{array}\left(\begin{array}{c}
N-1 \\
\ell
\end{array}\right)(x y)^{\ell}\right) \\
\times x^{b-a-1}(1-x)^{N-1} y^{b-1} \mathrm{~d} x \mathrm{~d} y \\
=N^{2} \int_{0}^{1} \int_{0}^{1} x^{b-a-1}(1-x)^{N-1} y^{b-1}(1-x y)^{N-1} \mathrm{~d} x \mathrm{~d} y .
\end{gathered}
$$


The computations can be pursued by performing the change of variables $(u, v)=(x, x y)$ in the above integral:

$$
\begin{aligned}
& \sum_{\ell=0}^{N-1} \frac{(-1)^{\ell}(N /(\ell+b))\left(\begin{array}{c}
N-1 \\
\ell
\end{array}\right)}{\left(\begin{array}{c}
\ell+b-a+N-1 \\
N
\end{array}\right)} \\
& \quad=N^{2} \iint_{\mathscr{D}^{+}} u^{-a-1}(1-u)^{N-1} v^{b-1}(1-v)^{N-1} \mathrm{~d} u \mathrm{~d} v .
\end{aligned}
$$

Putting (293) into (291) yields the expression of $\mathbb{P}\left\{\sigma_{b}^{+}<\sigma_{a}^{-}\right\}$ displayed in Theorem 43. The similar expression for $\mathbb{P}\left\{\sigma_{a}^{-}<\right.$ $\left.\sigma_{b}^{+}\right\}$holds true. Finally,

$$
\begin{aligned}
\mathbb{P}\left\{\sigma_{a b}\right. & <+\infty\} \\
& =\mathbb{P}\left\{\sigma_{a}^{-}<\sigma_{b}^{+}, \sigma_{a b}<+\infty\right\}+\mathbb{P}\left\{\sigma_{b}^{+}<\sigma_{a}^{-}, \sigma_{a b}<+\infty\right\} \\
& =\mathbb{P}\left\{\sigma_{a}^{-}<\sigma_{b}^{+}\right\}+\mathbb{P}\left\{\sigma_{b}^{+}<\sigma_{a}^{-}\right\} \\
& =K N^{2} \int_{0}^{1} \int_{0}^{1} u^{-a-1}(1-u)^{N-1} v^{b-1}(1-v)^{N-1} \mathrm{~d} u \mathrm{~d} v .
\end{aligned}
$$

The foregoing integral is quite elementary:

$$
\begin{aligned}
& \int_{0}^{1} \int_{0}^{1} u^{-a-1}(1-u)^{N-1} v^{b-1}(1-v)^{N-1} \mathrm{~d} u \mathrm{~d} v \\
& \quad=\int_{0}^{1} u^{-a-1}(1-u)^{N-1} \mathrm{~d} u \int_{0}^{1} v^{b-1}(1-v)^{N-1} \mathrm{~d} v \\
& \quad=B(N,-a) B(N, b) \\
& \quad=\frac{1}{N^{2}\left(\begin{array}{c}
N-a-1 \\
N
\end{array}\right)\left(\begin{array}{c}
b+N-1 \\
N
\end{array}\right)}=\frac{1}{K N^{2}},
\end{aligned}
$$

which entails that $\mathbb{P}\left\{\sigma_{a b}<+\infty\right\}=1$.

In the sequel, when considering $S_{a b}$, we will omit the condition $\sigma_{a b}<+\infty$.

Example 44. Let us have a look on the particular values 1, 2, 3 of $N$.

(i) Case $N=1$. In this case $S_{a b} \in\{a, b\}$ and

$$
\begin{gathered}
\mathbb{P}\left\{S_{a b}=a\right\}=\mathbb{P}\left\{\sigma_{a}^{-}<\sigma_{b}^{+}\right\}=\frac{b}{b-a}, \\
\mathbb{P}\left\{S_{a b}=b\right\}=\mathbb{P}\left\{\sigma_{b}^{+}<\sigma_{a}^{-}\right\}=-\frac{a}{b-a} .
\end{gathered}
$$

We retrieve one of the most well-known and important results for the ordinary random walk: this is the famous problem of the gambler's ruin!

(ii) Case $N=2$. In this case, the pseudorandom variables $\xi_{n}, n \in \mathbb{N}^{*}$, have two-valued upward jumps and two-valued downward jumps. Hence, the exit place must be either $a-1$, $a, b$ or $b+1: S_{a b} \in\{a-1, a, b, b+1\}$. We have that

$$
\begin{gathered}
\mathbb{P}\left\{S_{a b}=a-1\right\}=\frac{a b(b+1)}{(b-a+1)(b-a+2)}, \\
\mathbb{P}\left\{S_{a b}=a\right\}=-\frac{(a-1) b(b+1)}{(b-a)(b-a+1)}, \\
\mathbb{P}\left\{S_{a b}=b\right\}=\frac{a(a-1)(b+1)}{(b-a)(b-a+1)}, \\
\mathbb{P}\left\{S_{a b}=b+1\right\}=-\frac{a(a-1) b}{(b-a+1)(b-a+2)}, \\
\mathbb{P}\left\{\sigma_{a}^{-}<\sigma_{b}^{+}\right\}=\frac{b(b+1)(b-3 a+2)}{(b-a)(b-a+1)(b-a+2)}, \\
\mathbb{P}\left\{\sigma_{b}^{+}<\sigma_{a}^{-}\right\}=\frac{a(a-1)(3 b-a+2)}{(b-a)(b-a+1)(b-a+2)} .
\end{gathered}
$$

We can easily check that $\mathbb{P}\left\{S_{a b}=a\right\}+\mathbb{P}\left\{S_{a b}=a-1\right\}+\mathbb{P}\left\{S_{a b}=\right.$ $b\}+\mathbb{P}\left\{S_{a b}=b+1\right\}=1$ as well as $\mathbb{P}\left\{\sigma_{a}^{-}<\sigma_{b}^{+}\right\}+\mathbb{P}\left\{\sigma_{b}^{+}<\sigma_{a}^{-}\right\}=1$.

(iii) Case $N=3$. In this case $S_{a b} \in\{a-2, a-1, a, b, b+1, b+2\}$ and

$$
\begin{gathered}
\mathbb{P}\left\{S_{a b}=a-2\right\}=\frac{a(a-1) b(b+1)(b+2)}{(b-a+2)(b-a+3)(b-a+4)}, \\
\mathbb{P}\left\{S_{a b}=a-1\right\}=-\frac{a(a-2) b(b+1)(b+2)}{(b-a+1)(b-a+2)(b-a+3)}, \\
\mathbb{P}\left\{S_{a b}=a\right\}=\frac{(a-1)(a-2) b(b+1)(b+2)}{(b-a)(b-a+1)(b-a+2)} \\
\mathbb{P}\left\{S_{a b}=b\right\}=-\frac{a(a-1)(a-2)(b+1)(b+2)}{(b-a)(b-a+1)(b-a+2)} \\
\mathbb{P}\left\{S_{a b}=b+1\right\}=\frac{a(a-1)(a-2) b(b+2)}{(b-a+1)(b-a+2)(b-a+3)}, \\
\mathbb{P}\left\{\sigma_{b}^{-}<\sigma_{a}^{+}\right\} \\
=\frac{b(b+1)(b+2)\left(10 a^{2}-5 a b+b^{2}-25 a+7 b+12\right)}{(b-a)(b-a+1)(b-a+2)(b-a+3)(b-a+4)}, \\
\mathbb{P}\left\{\sigma_{b}^{+}<\sigma_{a}^{-}\right\} \\
a(a-1)(a-2)\left(a^{2}-5 a b+10 b^{2}-7 a+25 b+12\right) \\
(b-a)(b-a+1)(b-a+2)(b-a+3)(b-a+4)
\end{gathered}
$$

4.1.3. Pseudomoments of $S_{a b}$. Let us recall the notation we previously introduced in Section 3.1.3: $(i)_{n}=i(i-1)(i-$ $2) \cdots(i-n+1)$ for any $i \in \mathbb{Z}$ and any $n \in \mathbb{N}^{*}$ and $(i)_{0}=1$, as well as the conventions $1 / i !=0$ for any negative integer $i$ and $\sum_{k=i}^{j}=0$ if $i>j$. 
In this section, we compute several functionals related to the pseudomoments of $S_{a b}$. Namely, we provide formulae for $\mathbb{E}\left[S_{a b}\left(S_{a b}-b\right)_{n-1}\right]$ (Theorem 46), $\mathbb{E}\left[\left(S_{a b}\right)^{n}\right]$ (Corollary 47), and $\mathbb{E}\left[\left(S_{a b}-b\right)_{n}\right]$ (Theorem 48 ). This schedule may seem surprising; actually, we have been able to carry out the calculations by following this chronology.

Putting the identities $1 /(\stackrel{\ell+b-a+N-1}{N})=N \int_{0}^{1} x^{\ell+b-a-1}(1-$ $x)^{N-1} \mathrm{~d} x$ and $1 /(\ell+b)=\int_{0}^{1} y^{\ell+b-1} \mathrm{~d} y$ into the equality

$$
\begin{aligned}
\mathbb{E}\left[f\left(S_{a b}\right) \mathbb{1}_{\left\{S_{a b} \geq b\right\}}\right] & \\
& =K N \sum_{\ell=0}^{N-1} \frac{(-1)^{\ell}(f(\ell+b) /(\ell+b))\left(\begin{array}{c}
N-1 \\
\ell
\end{array}\right)}{\left(\begin{array}{c}
\ell+b-a+N-1 \\
N
\end{array}\right)},
\end{aligned}
$$

we immediately get the following integral representations for $\mathbb{E}\left[f\left(S_{a b}\right) \mathbb{1}_{\left\{S_{a b} \geq b\right\}}\right]$, and the analogous ones hold true for $\mathbb{E}\left[f\left(S_{a b}\right) \mathbb{1}_{\left\{S_{a b} \leq a\right\}}\right]$.

Theorem 45. For any function $f$ defined on $\mathscr{E}$,

$$
\begin{aligned}
& \mathbb{E}\left[f\left(S_{a b}\right) \mathbb{1}_{\left\{S_{a b} \geq b\right\}}\right] \\
& =K N^{2} \int_{0}^{1}\left[\sum_{\ell=0}^{N-1}(-1)^{\ell}\left(\begin{array}{c}
N-1 \\
\ell
\end{array}\right) \frac{f(\ell+b)}{\ell+b} x^{\ell}\right] \\
& \times x^{b-a-1}(1-x)^{N-1} \mathrm{~d} x \\
& =K N^{2} \int_{0}^{1} \int_{0}^{1}\left[\sum_{\ell=0}^{N-1}(-1)^{\ell}\left(\begin{array}{c}
N-1 \\
\ell
\end{array}\right) f(\ell+b)(x y)^{\ell}\right] \\
& \times x^{b-a-1}(1-x)^{N-1} y^{b-1} \mathrm{~d} x \mathrm{~d} y, \\
& \mathbb{E}\left[f\left(S_{a b}\right) \mathbb{1}_{\left\{S_{a b} \geq a\right\}}\right] \\
& =K N^{2} \int_{0}^{1}\left[\sum_{\ell=0}^{N-1}(-1)^{\ell}\left(\begin{array}{c}
N-1 \\
\ell
\end{array}\right) \frac{f(a-\ell)}{\ell-a} x^{\ell}\right] \\
& \times x^{b-a-1}(1-x)^{N-1} \mathrm{~d} x \\
& =K N^{2} \int_{0}^{1} \int_{0}^{1}\left[\sum_{\ell=0}^{N-1}(-1)^{\ell}\left(\begin{array}{c}
N-1 \\
\ell
\end{array}\right) f(a-\ell)(x y)^{\ell}\right] \\
& \times x^{b-a-1}(1-x)^{N-1} y^{-a-1} \mathrm{~d} x \mathrm{~d} y .
\end{aligned}
$$

In view of (300) and (302) and in order to compute the pseudomoments of $S_{a b}$, it is convenient to introduce the function $f_{n}$ defined by $f_{n}(i)=i(i-b)_{n-1}$ for any integers $i$ and $n$ such that $n \geq 1$. In particular, $f_{1}(i)=i$. We immediately see that, by choosing $f=f_{1}$ in Theorem 45 , quantities (300) and (302) are opposite. As a byproduct, $\mathbb{E}\left[S_{a b}\right]=0$. More generally, we have the results below.
Theorem 46. For any positive integer $n$,

$$
\begin{aligned}
& \mathbb{E}\left[S_{a b}\left(S_{a b}-b\right)_{n-1} \mathbb{1}_{\left\{S_{a b} \geq b\right\}}\right] \\
& =\left\{\begin{array}{c}
\frac{(-1)^{n-1}(K N /(2 N-n))(N ! /(N-n) !)}{\left(\begin{array}{c}
2 N+b-a-2 \\
2 N-n
\end{array}\right)} \\
\text { if } 1 \leq n \leq N, \\
\text { if } n \geq N+1,
\end{array}\right. \\
& \mathbb{E}\left[S_{a b}\left(S_{a b}-b\right)_{n-1} \mathbb{1}_{\left\{S_{a b} \leq a\right\}}\right] \\
& =\left\{\begin{array}{c}
\frac{(-1)^{n}(K N /(2 N-n))(N ! /(N-n) !)}{(2 N+b-a-2}{ }_{2 N-n} \text { if } 1 \leq n \leq N, \\
\text { if } N+1 \leq n \leq 2 N-1, \\
(-1)^{N+n-1} K N N !(n-N-1) !\left(\begin{array}{c}
n+b-a-2 \\
n-2 N
\end{array}\right)
\end{array}\right.
\end{aligned}
$$

In particular, for any $n \in\{1, \ldots, 2 N-1\}$,

$$
\mathbb{E}\left[S_{a b}\left(S_{a b}-b\right)_{n-1}\right]=0 .
$$

Proof. By (300), we get that

$$
\begin{aligned}
& \mathbb{E}\left[f_{n}\left(S_{a b}\right) \mathbb{1}_{\left\{S_{a b} \geq b\right\}}\right] \\
&=K N^{2} \int_{0}^{1}\left[\sum_{\ell=0}^{N-1}(-1)^{\ell}\left(\begin{array}{c}
N-1 \\
\ell
\end{array}\right)(\ell)_{n-1} x^{\ell+b-a-1}\right] \\
& \times(1-x)^{N-1} \mathrm{~d} x .
\end{aligned}
$$

By noticing that $(\ell)_{n-1}=0$ for $\ell \in\{0, \ldots, n-2\}$ and $\left(\begin{array}{c}N-1 \\ \ell\end{array}\right)(\ell)_{n-1}=\left(\begin{array}{c}N-n \\ \ell-n+1\end{array}\right)(N-1)_{n-1}$ for $\ell \geq n-1$, we obtain that

$$
\begin{aligned}
& \sum_{\ell=0}^{N-1}(-1)^{\ell}\left(\begin{array}{c}
N-1 \\
\ell
\end{array}\right)(\ell)_{n-1} x^{\ell} \\
& =(N-1)_{n-1} \mathbb{1}_{\{1 \leq n \leq N\}} \sum_{\ell=n-1}^{N-1}(-1)^{\ell}\left(\begin{array}{c}
N-n \\
\ell-n+1
\end{array}\right) x^{\ell} \\
& =(N-1)_{n-1} \mathbb{1}_{\{1 \leq n \leq N\}} x^{n-1} \sum_{\ell=0}^{N-n}(-1)^{\ell+n-1}\left(\begin{array}{c}
N-n \\
\ell
\end{array}\right) x^{\ell} \\
& = \begin{cases}(-1)^{n-1} \frac{(N-1) !}{(N-n) !} x^{n-1}(1-x)^{N-n} & \text { if } 1 \leq n \leq N, \\
0 & \text { if } n \geq N+1 .\end{cases}
\end{aligned}
$$


Hence, if $1 \leq n \leq N$,

$$
\begin{aligned}
\mathbb{E} & {\left[f_{n}\left(S_{a b}\right) \mathbb{1}_{\left\{S_{a b} \geq b\right\}}\right] } \\
& =(-1)^{n-1} K N^{2} \frac{(N-1) !}{(N-n) !} \int_{0}^{1} x^{n+b-a-2}(1-x)^{2 N-n-1} \mathrm{~d} x \\
& =(-1)^{n-1} \frac{K N N !}{(N-n) !} \frac{(n+b-a-2) !(2 N-n-1) !}{(2 N+b-a-2) !}
\end{aligned}
$$

and we arrive at (304). Moreover, if $n \geq N+1$ and $S_{a b} \geq b$, we have $S_{a b} \in\{b, b+1, \ldots, b+N-1\}$. Then, it is clear that $f_{n}\left(S_{a b}\right)=0$ and (304) still holds in this case.

On the other hand, by Theorem 43, we get that

$$
\begin{aligned}
\mathbb{E} & {\left[f_{n}\left(S_{a b}\right) \mathbb{1}_{\left\{S_{a b} \leq a\right\}}\right] } \\
& =\sum_{\ell=0}^{N-1} \mathbb{P}\left\{S_{a b}=a-\ell\right\} f_{n}(a-\ell) \\
& =K N \sum_{\ell=0}^{N-1} \frac{(-1)^{\ell-1}(a-b-\ell)_{n-1}\left(\begin{array}{c}
N-1 \\
\ell
\end{array}\right)}{\left(\begin{array}{c}
\ell+b-a+N-1 \\
N
\end{array}\right)} \\
& =(-1)^{n} K N N ! \sum_{\ell=0}^{N-1}(-1)^{\ell}\left(\begin{array}{c}
N-1 \\
\ell
\end{array}\right) \frac{(\ell+b-a+n-2) !}{(\ell+b-a+N-1) !} .
\end{aligned}
$$

For $n \leq N$, we can write that

$$
\begin{aligned}
& \frac{(\ell+b-a+n-2) !}{(\ell+b-a+N-1) !} \\
& \quad=\frac{1}{(N-n) !} \frac{1}{(\ell+b-a+N-1)\left(\begin{array}{l}
\ell+b-a+N-2 \\
N-n
\end{array}\right)} \\
& \quad=\frac{1}{(N-n) !} \int_{0}^{1} x^{\ell+n+b-a-2}(1-x)^{N-n} \mathrm{~d} x .
\end{aligned}
$$

Then,

$$
\begin{aligned}
\mathbb{E}\left[f_{n}\left(S_{a b}\right) \mathbb{1}_{\left\{S_{a b} \leq a\right\}}\right] \\
=(-1)^{n} \frac{K N N !}{(N-n) !} \\
\quad \times \int_{0}^{1} \sum_{\ell=0}^{N-1}(-1)^{\ell}\left(\begin{array}{c}
N-1 \\
\ell
\end{array}\right) x^{\ell+n+b-a-2}(1-x)^{N-n} \mathrm{~d} x \\
=(-1)^{n} \frac{K N N !}{(N-n) !} \int_{0}^{1} x^{n+b-a-2}(1-x)^{2 N-n-1} \mathrm{~d} x \\
=(-1)^{n} \frac{K N N !}{(N-n) !} \times \frac{(n+b-a-2) !(2 N-n-1) !}{(2 N+b-a-2) !}
\end{aligned}
$$

which proves (305). For $n \geq N+1$, we write instead that

$$
\frac{(\ell+b-a+n-2) !}{(\ell+b-a+N-1) !}=\left.\frac{\mathrm{d}^{n-N-1}}{\mathrm{~d} x^{n-N-1}}\left(x^{\ell+n+b-a-2}\right)\right|_{x=1} .
$$

Therefore,

$$
\begin{aligned}
\mathbb{E}[ & \left.f_{n}\left(S_{a b}\right) \mathbb{1}_{\left\{S_{a b} \leq a\right\}}\right] \\
= & (-1)^{n} K N N ! \\
& \times\left.\frac{\mathrm{d}^{n-N-1}}{\mathrm{~d} x^{n-N-1}}\left(\sum_{\ell=0}^{N-1}(-1)^{\ell}\left(\begin{array}{c}
N-1 \\
\ell
\end{array}\right) x^{\ell+n+b-a-2}\right)\right|_{x=1} \\
= & \left.(-1)^{n} K N N ! \frac{\mathrm{d}^{n-N-1}}{\mathrm{~d} x^{n-N-1}}\left(x^{n+b-a-2}(1-x)^{N-1}\right)\right|_{x=1} .
\end{aligned}
$$

If $N+1 \leq n \leq 2 N-1$, the above derivative vanishes since 1 is a root of multiplicity $N-1$ of the polynomial $x^{\ell+n+b-a-2}(1-x)^{N-1}$. Finally, for $n \geq 2 N$, we appeal to Leibniz rule for evaluating the derivative of interest:

$$
\begin{gathered}
\left.\frac{\mathrm{d}^{n-N-1}}{\mathrm{~d} x^{n-N-1}}\left(x^{n+b-a-2}(1-x)^{N-1}\right)\right|_{x=1} \\
=\sum_{k=0}^{n-N-1}(-1)^{k}\left(\begin{array}{c}
n-N-1 \\
k
\end{array}\right) \\
\quad \times \frac{(n+b-a-2) !}{(k+N+b-a-1) !}(N-1)_{k} \delta_{k, N-1} \\
=(-1)^{N-1}(N-1) ! \frac{(n+b-a-2) !}{(2 N+b-a-2) !}\left(\begin{array}{c}
n-N-1 \\
N-1
\end{array}\right) \\
=(-1)^{N-1}(n-N-1) !\left(\begin{array}{c}
n+b-a-2 \\
n-2 N
\end{array}\right) .
\end{gathered}
$$

This proves (305) in this case.

Corollary 47. For $n \in\{1, \ldots, 2 N-1\}$, the pseudo-moment of $S_{a b}$ of order $n$ vanishes:

$$
\mathbb{E}\left[\left(S_{a b}\right)^{n}\right]=0 .
$$

Moreover,

$$
\begin{aligned}
& \mathbb{E}\left[\left(S_{a b}\right)^{2 N}\right] \\
& \quad=-a(a-1) \cdots(a-N+1) b(b+1) \cdots(b+N-1) .
\end{aligned}
$$

Proof. As in the proof of Theorem 27, we appeal to the following argument: the polynomial $X^{n}$ is a linear combination of $f_{1}(X), \ldots, f_{n}(X)$. Then $\mathbb{E}\left[\left(S_{a b}\right)^{n}\right]$ can be written as a linear combination of $\mathbb{E}\left[f_{1}\left(S_{a b}\right)\right], \ldots, \mathbb{E}\left[f_{n}\left(S_{a b}\right)\right]$ which vanish when $1 \leq n \leq 2 N-1$. Thus, $\mathbb{E}\left[\left(S_{a b}\right)^{n}\right]=0$. The same argument entails that

$$
\mathbb{E}\left[\left(S_{a b}\right)^{2 N}\right]=\mathbb{E}\left[f_{2 N}\left(S_{a b}\right)\right]=(-1)^{N-1} K N !^{2}
$$

which proves (317). 
In the following theorem, we provide an integral representation for certain factorial pseudomoments of $\left(S_{a b}-a\right)$ and $\left(S_{a b}-b\right)$ which will be used in the next section.

Theorem 48. For any integer $n \in\{0, \ldots, N-1\}$,

$$
\begin{aligned}
& \mathbb{E}\left[\left(S_{a b}-b\right)_{n} \mathbb{1}_{\left\{S_{a b} \geq b\right\}}\right] \\
& =(-1)^{n} K N^{2}(N-1)_{n} \\
& \quad \times \iint_{\mathscr{D}^{+}} u^{-a-1}(1-u)^{N-1} v^{n+b-1}(1-v)^{N-n-1} \mathrm{~d} u \mathrm{~d} v \\
& \mathbb{E}\left[\left(a-S_{a b}\right)_{n} \mathbb{1}_{\left\{S_{a b} \leq a\right\}}\right] \\
& =(-1)^{n} K N^{2}(N-1)_{n} \\
& \quad \times \iint_{\mathscr{D}^{-}} u^{n-a-1}(1-u)^{N-n-1} v^{b-1}(1-v)^{N-1} \mathrm{~d} u \mathrm{~d} v .
\end{aligned}
$$

The above identities can be rewritten as

$$
\begin{aligned}
& \mathbb{E}\left[\left(\begin{array}{c}
S_{a b}-b \\
n
\end{array}\right) \mathbb{1}_{\left\{S_{a b} \geq b\right\}}\right] \\
& =(-1)^{n} K N^{2}\left(\begin{array}{c}
N-1 \\
n
\end{array}\right) \\
& \quad \times \iint_{\mathscr{D}^{+}} u^{-a-1}(1-u)^{N-1} v^{n+b-1}(1-v)^{N-n-1} \mathrm{~d} u \mathrm{~d} v, \\
& \mathbb{E}\left[\left(\begin{array}{c}
a-S_{a b} \\
n
\end{array}\right) \mathbb{1}_{\left\{S_{a b} \leq a\right\}}\right] \\
& =(-1)^{n} K N^{2}\left(\begin{array}{c}
N-1 \\
n
\end{array}\right) \\
& \quad \times \iint_{\mathscr{D}^{-}} u^{n-a-1}(1-u)^{N-n-1} v^{b-1}(1-v)^{N-1} \mathrm{~d} u \mathrm{~d} v .
\end{aligned}
$$

Proof. By (301), we have that

$$
\begin{aligned}
\mathbb{E}\left[\left(S_{a b}-b\right)_{n} \mathbb{1}_{\left\{S_{a b} \geq b\right\}}\right] \\
=K N^{2} \int_{0}^{1} \int_{0}^{1}\left[\sum_{\ell=0}^{N-1}(-1)^{\ell}\left(\begin{array}{c}
N-1 \\
\ell
\end{array}\right)(\ell)_{n}(x y)^{\ell}\right] \\
\quad \times x^{b-a-1}(1-x)^{N-1} y^{b-1} \mathrm{~d} x \mathrm{~d} y .
\end{aligned}
$$

The sum lying in the above integral can be easily calculated:

$$
\begin{aligned}
& \sum_{\ell=0}^{N-1}(-1)^{\ell}\left(\begin{array}{c}
N-1 \\
\ell
\end{array}\right)(\ell)_{n}(x y)^{\ell} \\
& \quad=(N-1)_{n} \sum_{\ell=n}^{N-1}(-1)^{\ell}\left(\begin{array}{c}
N-n-1 \\
\ell-n
\end{array}\right)(x y)^{\ell} \\
& \quad=(-1)^{n}(N-1)_{n}(x y)^{n}(1-x y)^{N-n-1} .
\end{aligned}
$$

Hence,

$$
\begin{aligned}
\mathbb{E} & {\left[\left(S_{a b}-b\right)_{n} \mathbb{1}_{\left\{S_{a b} \geq b\right\}}\right] } \\
= & (-1)^{n}(N-1)_{n} K N^{2} \\
& \times \int_{0}^{1} \int_{0}^{1} x^{n+b-a-1}(1-x)^{N-1} y^{n+b-1}(1-x y)^{N-n-1} \mathrm{~d} x \mathrm{~d} y .
\end{aligned}
$$

Performing the change of variables $(u, v)=(x, x y)$ in the foregoing integral immediately yields (319). Formula (320) can be deduced from (303) exactly in the same way.

4.2. Link with High-Order Finite-Difference Equations. Set $\Delta^{+} f(i)=f(i+1)-f(i)$ and $\Delta^{-} f(i)=f(i)-f(i-1)$ for any $i \in \mathbb{Z}$ and $\left(\Delta^{+}\right)^{j}=\underbrace{\Delta^{+} \circ \ldots \circ \Delta^{+}}_{j \text { times }}$ and $\left(\Delta^{-}\right)^{j}=\underbrace{\Delta^{-} \circ \ldots \circ \Delta^{-}}_{j \text { times }}$ for any $j \in \mathbb{N}^{*}$. Set also $\left(\Delta^{+}\right)^{0} f=\left(\Delta^{-}\right)^{0} f=f$. The quantities $\left(\Delta^{+}\right)^{j}$ and $\left(\Delta^{-}\right)^{j}$ are the iterated forward and backward finitedifference operators given by

$$
\begin{aligned}
\left(\Delta^{+}\right)^{j} f(i) & =\sum_{k=0}^{j}(-1)^{j+k}\left(\begin{array}{l}
j \\
k
\end{array}\right) f(i+k), \\
\left(\Delta^{-}\right)^{j} f(i) & =\sum_{k=0}^{j}(-1)^{k}\left(\begin{array}{l}
j \\
k
\end{array}\right) f(i-k) .
\end{aligned}
$$

Conversely, $f(i+k)$ and $f(i-k)$ can be expressed by means of $\left(\Delta^{+}\right)^{j} f(i),\left(\Delta^{-}\right)^{j} f(i), 0 \leq j \leq k$, according to

$$
\begin{gathered}
f(i+k)=\sum_{j=0}^{k}\left(\begin{array}{l}
k \\
j
\end{array}\right)\left(\Delta^{+}\right)^{j} f(i), \\
f(i-k)=\sum_{j=0}^{k}(-1)^{j}\left(\begin{array}{l}
k \\
j
\end{array}\right)\left(\Delta^{-}\right)^{j} f(i) .
\end{gathered}
$$

We have the following expression for any functional of the pseudorandom variable $S_{a b}$.

Theorem 49. One has, for any function $f$ defined on $\mathscr{E}$, that

$$
\mathbb{E}\left[f\left(S_{a b}\right)\right]=\sum_{j=0}^{N-1} I_{a b, j}^{-}\left(\Delta^{-}\right)^{j} f(a)+\sum_{j=0}^{N-1} I_{a b, j}^{+}\left(\Delta^{+}\right)^{j} f(b)
$$


with

$$
\begin{aligned}
I_{a b, j}^{-}= & K N^{2}\left(\begin{array}{c}
N-1 \\
j
\end{array}\right) \\
& \times \iint_{\mathscr{D}^{-}} u^{j-a-1}(1-u)^{N-j-1} v^{b-1}(1-v)^{N-1} \mathrm{~d} u \mathrm{~d} v, \\
I_{a b, j}^{+}= & (-1)^{j} K N^{2}\left(\begin{array}{c}
N-1 \\
j
\end{array}\right) \\
& \times \iint_{\mathscr{D}^{+}} u^{-a-1}(1-u)^{N-1} v^{j+b-1}(1-v)^{N-j-1} \mathrm{~d} u \mathrm{~d} v .
\end{aligned}
$$

Proof. By (327), we see that

$$
\begin{aligned}
\mathbb{E}\left[f\left(S_{a b}\right)\right]= & \mathbb{E}\left(\mathbb{1}_{\left\{S_{a b} \leq a\right\}} \sum_{j=0}^{a-S_{a b}}(-1)^{j}\left(\begin{array}{c}
a-S_{a b} \\
j
\end{array}\right)\left(\Delta^{-}\right)^{j} f(a)\right) \\
& +\mathbb{E}\left(\mathbb{1}_{\left\{S_{a b} \geq b\right\}} \sum_{j=0}^{S_{a b}-b}\left(\begin{array}{c}
S_{a b}-b \\
j
\end{array}\right)\left(\Delta^{+}\right)^{j} f(b)\right) \\
= & \sum_{j=0}^{N-1}(-1)^{j} \mathbb{E}\left[\mathbb{1}_{\left\{S_{a b} \leq a\right\}}\left(\begin{array}{c}
a-S_{a b} \\
j
\end{array}\right)\right]\left(\Delta^{-}\right)^{j} f(a) \\
& +\sum_{j=0}^{N-1} \mathbb{E}\left[\mathbb{1}_{\left\{S_{a b} \geq b\right\}}\left(\begin{array}{c}
S_{a b}-b \\
j
\end{array}\right)\right]\left(\Delta^{+}\right)^{j} f(b)
\end{aligned}
$$

which immediately yields (328) thanks to (321) and (322).

Corollary 50. The generating function of $S_{a b}$ is given by

$$
\mathbb{E}\left(\zeta^{S_{a b}}\right)=\zeta^{a} \sum_{j=0}^{N-1} I_{a b, j}^{-}\left(1-\frac{1}{\zeta}\right)^{j}+\zeta^{b} \sum_{j=0}^{N-1} I_{a b, j}^{+}(\zeta-1)^{j} .
$$

Proof. Let us apply Theorem 49 to the function $f(i)=\zeta^{i}$ for which we plainly have $\left(\Delta^{+}\right)^{j} f(i)=\zeta^{i}(\zeta-1)^{j}$ and $\left(\Delta^{-}\right)^{j} f(i)=$ $\zeta^{i}(1-1 / \zeta)^{j}$. This immediately yields (331).

Of special interest is the case when the starting point of the pseudorandom walk is some point $x \in \mathbb{Z}$. By translating $a, b$ into $a-x, b-x$ and the function $f$ into the shifted function $f(\cdot+x)$, we have that

$$
\begin{aligned}
\mathbb{E}_{x}\left[f\left(S_{a b}\right)\right]= & \mathbb{E}\left[f\left(x+S_{a-x, b-x}\right)\right] \\
= & \sum_{j=0}^{N-1} I_{a-x, b-x, j}^{-}\left(\Delta^{-}\right)^{j} f(a) \\
& +\sum_{j=0}^{N-1} I_{a-x, b-x, j}^{+}\left(\Delta^{+}\right)^{j} f(b) .
\end{aligned}
$$

More precisely, we have the following result.
Theorem 51. One has, for any function $f$ defined on $\mathscr{E}$, that

$$
\begin{aligned}
\mathbb{E}_{x}\left[f\left(S_{a b}\right)\right]= & \sum_{j=0}^{N-1} P_{a b, j}^{-}(x)\left(\Delta^{-}\right)^{j} f(a) \\
& +\sum_{j=0}^{N-1} P_{a b, j}^{+}(x)\left(\Delta^{+}\right)^{j} f(b),
\end{aligned}
$$

where $P_{a b, j}^{-}$and $P_{a b, j}^{+}, 0 \leq j \leq N-1$, are polynomials of degree not greater than $2 N-1$ characterized, for any $k \in\{0, \ldots$, $N-1\}$, by

$$
\begin{array}{r}
\left(\Delta^{-}\right)^{k} P_{a b, j}^{-}(a)=\left(\Delta^{+}\right)^{k} P_{a b, j}^{+}(b)=\delta_{j k}, \\
\left(\Delta^{-}\right)^{k} P_{a b, j}^{+}(a)=\left(\Delta^{+}\right)^{k} P_{a b, j}^{-}(b)=0 .
\end{array}
$$

Proof. By setting $P_{a b, j}^{-}(x)=I_{a-x, b-x, j}^{-}$and $P_{a b, j}^{+}(x)=I_{a-x, b-x, j}^{+}$, (330) immediately yields (333). By observing that $P_{a b, j}^{-}(x)=$ $(-1)^{j} P_{a b, j}^{+}(a+b-x)$, it is enough to work with, for example, $P_{a b, j}^{+}$. Coming back to the proof of Theorem 49 and appealing to Theorem 43 , we write that

$$
\begin{aligned}
P_{a b, j}^{+}(x)= & \mathbb{E}_{x}\left[\mathbb{1}_{\left\{S_{a b} \geq b\right\}}\left(\begin{array}{c}
S_{a b}-b \\
j
\end{array}\right)\right] \\
= & \sum_{m=j}^{N-1}\left(\begin{array}{c}
m \\
j
\end{array}\right) \mathbb{P}_{x}\left\{S_{a b}=b+m\right\} \\
= & \frac{1}{(N-1) ! N !} \\
& \times \sum_{m=j}^{N-1}(-1)^{m}\left[\frac{\left(\begin{array}{c}
m \\
j
\end{array}\right)\left(\begin{array}{c}
N-1 \\
m
\end{array}\right)}{\left(\begin{array}{c}
m+b-a+N-1 \\
N
\end{array}\right)}\right] \widetilde{K}_{m}(x),
\end{aligned}
$$

where, for any $m \in\{0, \ldots, N-1\}$,

$$
\begin{aligned}
\widetilde{K}_{m}(x) & =\frac{\left(\prod_{k=0}^{N-1}[(x-a+k)(b-x+k)]\right)}{(b-x+m)} \\
& =\prod_{k=0}^{N-1}(x-a+k) \prod_{\substack{0 \leq k \leq N-1 \\
k \neq m}}(b-x+k) .
\end{aligned}
$$

The expression $\widetilde{K}_{m}(x)$ defines a polynomial of the variable $x$ of degree $2 N-1$, so $P_{a b, j}^{+}$is a polynomial of degree not greater than $2 N-1$.

It is obvious that $\widetilde{K}_{m}(a-\ell)=0$ for $\ell, m \in\{0, \ldots, N-1\}$. Then, $P_{a b, j}^{+}(a-\ell)=0$ which implies that $\left(\Delta^{-}\right)^{k} P_{a b, j}^{+}(a)=0$ for any $k \in\{0, \ldots, N-1\}$. Now, let us evaluate $P_{a b, j}^{+}(b+\ell)$ for $\ell \in\{0, \ldots, N-1\}$. We plainly have that $\widetilde{K}_{m}(b+\ell)=0$ for $\ell \neq m$ and that

$$
\widetilde{K}_{\ell}(b+\ell)=\frac{(-1)^{\ell}(N-1) ! N !\left({ }^{\ell+b-a+N-1}\right)}{\left(\begin{array}{c}
N-1 \\
\ell
\end{array}\right)} .
$$


By putting this into (335), we get that

$$
P_{a b, j}^{+}(b+\ell)=\left(\begin{array}{l}
\ell \\
j
\end{array}\right) \mathbb{1}_{\{j \leq \ell\}} .
$$

Next, we obtain, for any $k \in\{0, \ldots, N-1\}$, that

$$
\begin{aligned}
\left(\Delta^{+}\right)^{k} P_{a b, j}^{+}(b) & =\sum_{\ell=0}^{k}(-1)^{k+\ell}\left(\begin{array}{l}
k \\
\ell
\end{array}\right) P_{a b, j}^{+}(b+\ell) \\
& =\sum_{\ell=j}^{k}(-1)^{k+\ell}\left(\begin{array}{l}
k \\
\ell
\end{array}\right)\left(\begin{array}{l}
\ell \\
j
\end{array}\right) \\
& =(-1)^{j+k}\left(\begin{array}{c}
k \\
j
\end{array}\right) \sum_{\ell=0}^{k-j}(-1)^{\ell}\left(\begin{array}{c}
k-j \\
\ell
\end{array}\right)=\delta_{j k} .
\end{aligned}
$$

The proof of Theorem 51 is finished.

Example 52. In the case where $N=2$, (333) writes as

$$
\begin{aligned}
\mathbb{E}_{x}\left[f\left(S_{a b}\right)\right]= & P_{a b, 0}^{-}(x) f(a)+P_{a b, 1}^{-}(x) \Delta^{-} f(a) \\
& +P_{a b, 0}^{+}(x) f(b)+P_{a b, 1}^{+}(x) \Delta^{+} f(b)
\end{aligned}
$$

with

$$
\begin{aligned}
& P_{a b, 0}^{-}(x)=\frac{(x-b)(x-b-1)(2 x-3 a+b+2)}{(b-a)(b-a+1)(b-a+2)}, \\
& P_{a b, 1}^{-}(x)=\frac{(x-a)(x-b)(x-b-1)}{(b-a+1)(b-a+2)}, \\
& P_{a b, 0}^{+}(x)=-\frac{(x-a)(x-a+1)(2 x+a-3 b-2)}{(b-a)(b-a+1)(b-a+2)}, \\
& P_{a b, 1}^{+}(x)=\frac{(x-a)(x-a+1)(x-b)}{(b-a+1)(b-a+2)} .
\end{aligned}
$$

Below, we state a strong pseudo-Markov property related to time $\sigma_{a b}$.

Theorem 53. One has, for any function $f$ defined on $\mathbb{Z}$ and any $n \in \mathbb{N}$, that

$$
\begin{aligned}
\mathbb{E}_{x}\left[f\left(S_{\sigma_{a b}+n}\right)\right]= & \sum_{j=0}^{N-1} P_{a b, j}^{-}(x)\left(\Delta^{-}\right)^{j} \mathbb{E}_{a}\left[f\left(S_{n}\right)\right] \\
& +\sum_{j=0}^{N-1} P_{a b, j}^{+}(x)\left(\Delta^{+}\right)^{j} \mathbb{E}_{b}\left[f\left(S_{n}\right)\right] .
\end{aligned}
$$

In (342), the operators $\left(\Delta^{-}\right)^{j}$ and $\left(\Delta^{+}\right)^{j}$ act on the variables a and $b$.

Proof. Formula (342) can be proved exactly in the same way as (206): by setting $g(x)=\mathbb{E}_{x}\left[f\left(S_{n}\right)\right]$, we have that $\mathbb{E}_{x}\left[f\left(S_{\sigma_{a b}+n}\right)\right]=\mathbb{E}_{x}\left[g\left(S_{a b}\right)\right]$. This proves (342) thanks to (333).

Example 54. Below, we display the form of (342) for the particular values 1,2 of $N$. (i) For $N=1$, (206) reads as

$$
\mathbb{E}_{x}\left[f\left(S_{\sigma_{a b}+n}\right)\right]=\frac{b-x}{b-a} \mathbb{E}_{a}\left[f\left(S_{n}\right)\right]+\frac{x-a}{b-a} \mathbb{E}_{b}\left[f\left(S_{n}\right)\right]
$$

which is of course well known! This is the strong Markov property for the ordinary random walk.

(ii) For $N=2$, (206) reads as

$$
\begin{aligned}
\mathbb{E}_{x}[ & \left.f\left(S_{\sigma_{a b}+n}\right)\right] \\
= & \frac{(x-b)(x-b-1)(2 x-3 a+b+2)}{(b-a)(b-a+1)(b-a+2)} \mathbb{E}_{a}\left[f\left(S_{n}\right)\right] \\
& +\frac{(x-a)(x-b)(x-b-1)}{(b-a+1)(b-a+2)} \Delta^{-} \mathbb{E}_{a}\left[f\left(S_{n}\right)\right] \\
& -\frac{(x-a)(x-a+1)(2 x+a-3 b-2)}{(b-a)(b-a+1)(b-a+2)} \mathbb{E}_{b}\left[f\left(S_{n}\right)\right] \\
& +\frac{(x-a)(x-a+1)(x-b)}{(b-a+1)(b-a+2)} \Delta^{+} \mathbb{E}_{b}\left[f\left(S_{n}\right)\right] \\
= & \frac{(x-a+1)(x-b)(x-b-1)}{(b-a)(b-a+1)} \mathbb{E}_{a}\left[f\left(S_{n}\right)\right] \\
& -\frac{(x-a)(x-b)(x-b-1)}{(b-a+1)(b-a+2)} \mathbb{E}_{a-1}\left[f\left(S_{n}\right)\right] \\
& -\frac{(x-a)(x-a+1)(x-b-1)}{(b-a)(b-a+1)} \mathbb{E}_{b}\left[f\left(S_{n}\right)\right] \\
& +\frac{(x-a)(x-a+1)(x-b)}{(b-a+1)(b-a+2)} \mathbb{E}_{b+1}\left[f\left(S_{n}\right)\right] .
\end{aligned}
$$

Now, we consider the discrete Laplacian $\Delta=\Delta^{+} \circ \Delta^{-}=$ $\Delta^{-} \circ \Delta^{+}$. It is explicitly defined by $\Delta f(i)=f(i+1)-2 f(i)+$ $f(i-1)$. Let us introduce the iterated Laplacian $\Delta^{N}=\left(\Delta^{+}\right)^{N}$ 。 $\left(\Delta^{-}\right)^{N}=\left(\Delta^{-}\right)^{N} \circ\left(\Delta^{+}\right)^{N}$. We compute $\Delta^{N} f(i)$ for any function $f$ and any $i \in \mathbb{Z}$ :

$\Delta^{N} f(i)$

$$
\begin{aligned}
& =\left(\Delta^{-}\right)^{N}\left(\sum_{j=0}^{N}(-1)^{j+N}\left(\begin{array}{c}
N \\
j
\end{array}\right) f(\cdot+j)\right)(i) \\
& =\sum_{j=0}^{N}(-1)^{j+N}\left(\begin{array}{c}
N \\
j
\end{array}\right)\left(\sum_{k=0}^{N}(-1)^{k}\left(\begin{array}{l}
N \\
k
\end{array}\right) f(i+j-k)\right) \\
& =(-1)^{N} \sum_{0 \leq j, k \leq N}(-1)^{j-k}\left(\begin{array}{c}
N \\
j
\end{array}\right)\left(\begin{array}{c}
N \\
k
\end{array}\right) f(i+j-k) \\
& =(-1)^{N} \sum_{\ell=-N}^{N}(-1)^{\ell}\left(\sum_{k=(-\ell) \vee 0}^{(N-\ell) \wedge N}\left(\begin{array}{c}
N \\
k
\end{array}\right)\left(\begin{array}{c}
N \\
k+\ell
\end{array}\right)\right) f(i+\ell) .
\end{aligned}
$$


By using the elementary identity $\sum_{k=(\ell-q) \vee 0}^{p \wedge \ell}\left(\begin{array}{l}p \\ k\end{array}\right)\left(\begin{array}{c}q \\ \ell-k\end{array}\right)=$ $\left(\begin{array}{c}p+q \\ \ell\end{array}\right)$, we get that

$$
\begin{aligned}
\sum_{k=(-\ell) \vee 0}^{(N-\ell) \wedge N}\left(\begin{array}{l}
N \\
k
\end{array}\right)\left(\begin{array}{c}
N \\
k+\ell
\end{array}\right) & =\sum_{k=(-\ell) \vee 0}^{(N-\ell) \wedge N}\left(\begin{array}{l}
N \\
k
\end{array}\right)\left(\begin{array}{c}
N \\
N-k-\ell
\end{array}\right) \\
& =\left(\begin{array}{c}
2 N \\
\ell+N
\end{array}\right) .
\end{aligned}
$$

As a result, we obtain the expression of $\Delta^{N} f(i)$ announced in the introduction, namely,

$$
\Delta^{N} f(i)=\sum_{\ell=-N}^{N}(-1)^{\ell+N}\left(\begin{array}{c}
2 N \\
\ell+N
\end{array}\right) f(i+\ell) .
$$

Example 55. Fix a nonnegative integer $j$ and put $f_{j}(i)=(i)_{j}$ for any $i \in \mathbb{Z}$. It is plain that, if $k \leq j,\left(\Delta^{+}\right)^{k} f_{j}(i)=$ $(j)_{k} f_{j-k}(i),\left(\Delta^{-}\right)^{k} f_{j}(i)=(j)_{k} f_{j-k}(i-1)$ and if $k>j$, $\left(\Delta^{+}\right)^{k} f_{j}(i)=\left(\Delta^{-}\right)^{k} f_{j}(i)=0$. Therefore, if $2 k \leq j, \Delta^{k} f_{j}(i)=$ $(j)_{k}(j-k)_{k} f_{j-2 k}(i-1)$ and if $2 k>j, \Delta^{k} f_{j}(i)=0$. By using a linear algebra argument, we deduce that $\Delta^{N} P=0$ for any polynomial $P$ of degree not greater than $2 N-1$. As a byproduct,

$$
\Delta^{N} P_{a b, j}^{+}=\Delta^{N} P_{a b, j}^{-}=0 .
$$

Now, the main link between time $\sigma_{a b}$ and finite-difference equations is the following one.

Theorem 56. Let $\varphi$ be a function defined on $\mathscr{E}$. The function $\Phi$ defined on $\mathbb{Z}$ by $\Phi(x)=\mathbb{E}_{x}\left[\varphi\left(S_{a b}\right)\right]$ is the solution to the discrete Lauricella's problem

$$
\begin{array}{cc}
\Delta^{N} \Phi(x)=0 & \text { for } x \in \mathbb{Z}, \\
\left(\Delta^{-}\right)^{k} \Phi(a)=\left(\Delta^{-}\right)^{k} \varphi(a) & \text { for } j \in\{0, \ldots, N-1\}, \\
\left(\Delta^{+}\right)^{k} \Phi(b)=\left(\Delta^{+}\right)^{k} \varphi(b) & \text { for } j \in\{0, \ldots, N-1\} .
\end{array}
$$

Proof. By (333) we write that

$$
\Phi(x)=\sum_{j=0}^{N-1} P_{a b, j}^{-}(x)\left(\Delta^{-}\right)^{j} \varphi(a)+\sum_{j=0}^{N-1} P_{a b, j}^{+}(x)\left(\Delta^{+}\right)^{j} \varphi(b) .
$$

With this representation at hand, identities (334) and (348) immediately yield equations (349).

4.3. Joint Pseudodistribution of $\left(\tau_{a b}, X_{a b}\right)$. As in Section 3.3, we choose for the family $\left(\left(X_{t}^{\varepsilon}\right)_{t \geq 0}\right)_{\varepsilon>0}$ the pseudoprocesses defined, for any $\varepsilon>0$, by

$$
X_{t}^{\varepsilon}=\varepsilon S_{\left\lfloor t / \varepsilon^{2 N}\right\rfloor}, \quad t \geq 0
$$

and for the pseudoprocess $\left(X_{t}\right)_{t \geq 0}$ the pseudo-Brownian motion. In Definition 34, we choose for $I$ the interval $(a, b)$; then $\tau_{I}^{\varepsilon}=\tau_{a b}^{\varepsilon}, X_{I}^{\varepsilon}=X_{a b}^{\varepsilon}$ and $\tau_{I}=\tau_{a b}, X_{I}=X_{a b}$. Set $a_{\varepsilon}=\lfloor a / \varepsilon\rfloor$ and $b_{\varepsilon}=\lfloor b / \varepsilon\rfloor$, where $\lfloor\cdot\rfloor$ and $\lceil\cdot\rceil$, respectively stand for the usual floor and ceiling functions. We have $\tau_{a b}^{\varepsilon}=\varepsilon^{2 N} \sigma_{a_{\varepsilon}, b_{\varepsilon}}$ and $X_{a b}^{\varepsilon}=\varepsilon S_{a_{\varepsilon}, b_{\varepsilon}}$.

Theorem 57. The following convergence holds:

$$
\left(\tau_{a b}^{\varepsilon}, X_{a b}^{\varepsilon}\right) \underset{\varepsilon \rightarrow 0^{+}}{\longrightarrow}\left(\tau_{a b}, X_{a b}\right)
$$

where, for any $\lambda>0$ and any $\mu \in \mathbb{R}$,

$$
\begin{aligned}
& \mathbb{E}\left(\mathrm{e}^{-\lambda \tau_{a b}+i \mu X_{a b}} \mathbb{1}_{\left\{\tau_{a b}<+\infty\right\}}\right) \\
& =\mathrm{e}^{i \mu a} \sum_{k=1}^{N} \frac{\mathbf{D}_{k}^{-}(\lambda, \mu)}{\mathbf{D}(\lambda)}+\mathrm{e}^{i \mu b} \sum_{k=1}^{N} \frac{\mathbf{D}_{k}^{+}(\lambda, \mu)}{\mathbf{D}(\lambda)} .
\end{aligned}
$$

In the foregoing formula, $\mathbf{D}(\lambda), \mathbf{D}_{k}^{-}(\lambda, \mu)$, and $\mathbf{D}_{k}^{+}(\lambda, \mu)$ are the respective determinants

$$
\begin{gathered}
\left|\begin{array}{cccccccc}
1 & \varphi_{1} & \cdots & \varphi_{1}^{N-1} & \mathrm{e}^{-\varphi_{1}} \sqrt[2 N]{\lambda / c}(b-a) & \mathrm{e}^{-\varphi_{1} \sqrt[2 N]{\lambda / c}(b-a)} \varphi_{1} & \cdots & \mathrm{e}^{-\varphi_{1} \sqrt[2 N]{\lambda / c}(b-a)} \varphi_{1}^{N-1} \\
\vdots & \vdots & & \vdots & \vdots & \vdots & & \vdots \\
1 & \varphi_{2 N} & \cdots & \varphi_{2 N}^{N-1} & \mathrm{e}^{-\varphi_{2 N} \sqrt[2 N]{\lambda / c}(b-a)} & \mathrm{e}^{-\varphi_{2 N} \sqrt[2 N]{\lambda / c}(b-a)} \varphi_{2 N} & \cdots & \mathrm{e}^{-\varphi_{2 N} \sqrt[2 N]{\lambda / c}(b-a)} \varphi_{2 N}^{N-1}
\end{array}\right|, \\
\left|\begin{array}{cccccccc}
1 & \varphi_{1} & \cdots & \varphi_{1}^{N-1} & \mathrm{e}^{-\varphi_{1}} \sqrt[2 N]{\lambda / c}(b-a) & \mathrm{e}^{-\varphi_{1} \sqrt[2 N]{\lambda / c}(b-a)} \varphi_{1} & \cdots & \mathrm{e}^{-\varphi_{1} \sqrt[2 N]{\lambda / c}(b-a)} \varphi_{1}^{N-1} \\
\vdots & \vdots & & \vdots & \vdots & \vdots & & \vdots \\
1 & \varphi_{k-1} & \cdots & \varphi_{k-1}^{N-1} & \mathrm{e}^{-\varphi_{k-1} \sqrt[2 N]{\lambda / c}(b-a)} & \mathrm{e}^{-\varphi_{k-1} \sqrt[2 N]{\lambda / c}(b-a)} \varphi_{k-1} & \cdots & \mathrm{e}^{-\varphi_{k-1} \sqrt[2 N]{\lambda / c}(b-a)} \varphi_{k-1}^{N-1} \\
1 & \delta & \cdots & \delta^{N-1} & 0 & 0 & \cdots & 0 \\
1 & \varphi_{k+1} & \cdots & \varphi_{k+1}^{N-1} & \mathrm{e}^{-\varphi_{k+1}} \sqrt[2 N]{\lambda / c}(b-a) & \mathrm{e}^{-\varphi_{k+1} \sqrt[2 N]{\lambda / c}(b-a)} \varphi_{k+1} & \cdots & \mathrm{e}^{-\varphi_{k+1} \sqrt[2 N]{\lambda / c}(b-a)} \varphi_{k+1}^{N-1} \\
\vdots & \vdots & & \vdots & \vdots & \vdots & & \vdots \\
1 & \varphi_{2 N} & \cdots & \varphi_{2 N}^{N-1} & \mathrm{e}^{-\varphi_{2 N} \sqrt[2 N]{\lambda / c}(b-a)} & \mathrm{e}^{-\varphi_{2 N} \sqrt[2 N]{\lambda / c}(b-a)} \varphi_{2 N} & \cdots & \mathrm{e}^{-\varphi_{2 N} \sqrt[2 N]{\lambda / c}(b-a)} \varphi_{2 N}^{N-1}
\end{array}\right|,
\end{gathered}
$$




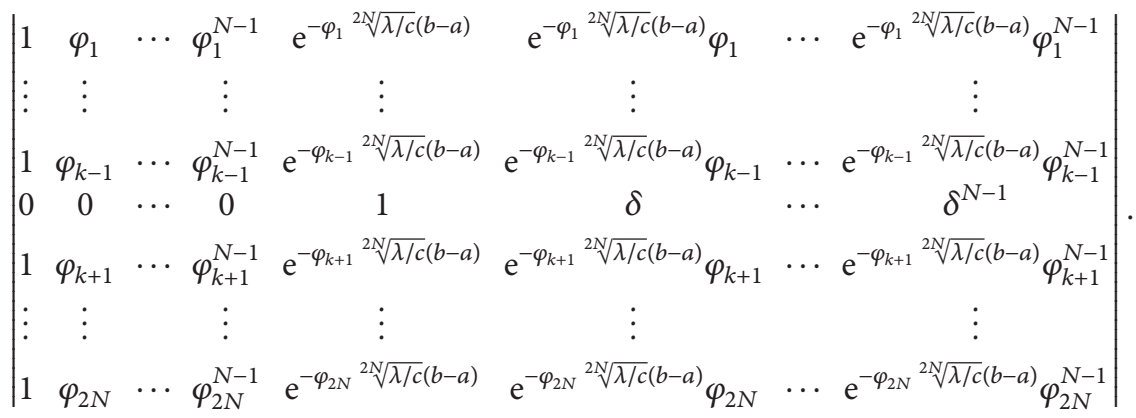

In the two last determinants, we have put $\delta=-i \mu / \sqrt[2 N]{\lambda / c}$.

In Theorem 57, we obtain the joint pseudodistribution of $\left(\tau_{a b}, X_{a b}\right)$ characterized by its Laplace-Fourier transform. This is a new result for pseudo-Brownian motion that we will develop in a forthcoming paper [14].

Proof. By Definition 34 and Theorem 39, we have that

$$
\begin{aligned}
& \mathbb{E}\left(\mathrm{e}^{-\lambda \tau_{a b}+i \mu X_{a b}} \mathbb{1}_{\left\{\tau_{a b}<+\infty\right\}}\right) \\
& =\lim _{\varepsilon \rightarrow 0^{+}} \mathbb{E}\left(\mathrm{e}^{-\lambda \tau_{a b}^{\varepsilon}+i \mu X_{a b}^{\varepsilon}} \mathbb{1}_{\left\{\tau_{a b}^{\varepsilon}<+\infty\right\}}\right)
\end{aligned}
$$

$$
\begin{aligned}
& =\lim _{\varepsilon \rightarrow 0^{+}} \mathbb{E}\left(\mathrm{e}^{-\lambda \varepsilon^{2 N} \sigma_{a_{\varepsilon}, b_{\varepsilon}}+i \mu \varepsilon S_{a_{\varepsilon}, b_{\varepsilon}}} \mathbb{1}_{\left\{\sigma_{a_{\varepsilon}, b_{\varepsilon}}<+\infty\right\}}\right) \\
& =\lim _{\varepsilon \rightarrow 0^{+}} \sum_{k=1}^{2 N} \widetilde{L}_{k}\left(\mathrm{e}^{-\lambda \varepsilon^{2 N}}, \mathrm{e}^{i \mu \varepsilon}\right)\left(\mathrm{e}^{i \mu \varepsilon} v_{k}(\lambda, \varepsilon)\right)^{a_{\varepsilon}-N} \\
& =\mathrm{e}^{i \mu a} \sum_{k=1}^{2 N} \lim _{\varepsilon \rightarrow 0^{+}} \widetilde{L}_{k}\left(\mathrm{e}^{-\lambda \varepsilon^{2 N}}, \mathrm{e}^{i \mu \varepsilon}\right) v_{k}(\lambda, \varepsilon)^{a_{\varepsilon}} .
\end{aligned}
$$

Recall that $\widetilde{L}_{k}(z, \zeta)=D_{k}(z, \zeta) / D(z)$ and that the quantities $D$ and $D_{k}$ are expressed by means of the determinant

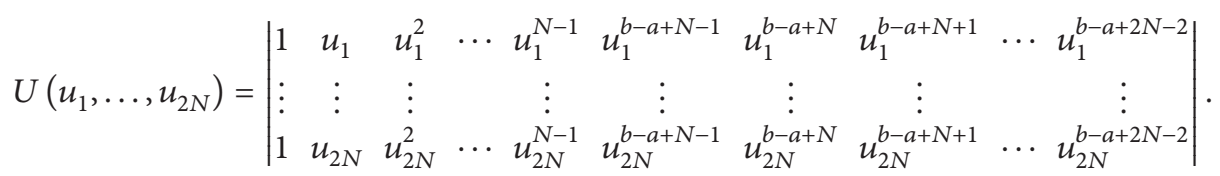

By replacing the columns labeled as $C_{j}, 1 \leq j \leq 2 N$, by the linear combinations $C_{j}^{\prime}=\sum_{k=0}^{j}(-1)^{j+k}\left(\begin{array}{l}j \\ k\end{array}\right) C_{k}$ if $1 \leq j \leq N$, and $C_{j}^{\prime}=\sum_{k=N}^{j}(-1)^{j+k}\left(\begin{array}{l}j \\ k\end{array}\right) C_{k}$ if $N+1 \leq j \leq 2 N$, the foregoing determinant remains invariant and can be rewritten as

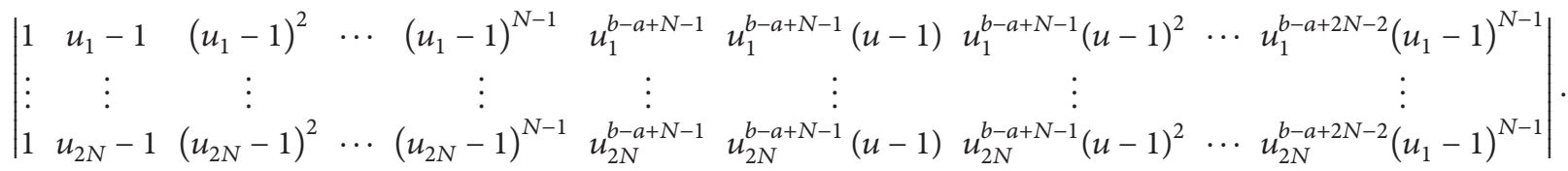

Then, by replacing the $u_{j}$ 's by $u_{j}(\lambda, \varepsilon), b-a$ by $b_{\varepsilon}-a_{\varepsilon}$ and by using the asymptotics $\left(u_{j}(\lambda, \varepsilon)-1\right)^{k} \underset{\varepsilon \rightarrow 0^{+}}{\sim}\left(-\varphi_{j} \sqrt[2 N]{\lambda / c}\right)^{k} \varepsilon^{k}$ and $u_{j}(\lambda, \varepsilon)^{b_{\varepsilon}-a_{\varepsilon}+N-1} \underset{\varepsilon \rightarrow 0^{+}}{\rightarrow} e^{-\varphi_{j} \sqrt[2 N]{\lambda / c}(b-a)}$ coming from (119), we get that 


$$
\begin{aligned}
& D\left(\mathrm{e}^{-\lambda \varepsilon^{2 N}}\right)=U\left(u_{1}(\lambda, \varepsilon), \ldots, u_{2 N}(\lambda, \varepsilon)\right) \\
& \underset{\varepsilon \rightarrow 0^{+}}{\sim} \mid \begin{array}{ccccc}
1 & \left(-\varphi_{1} \sqrt[2 N]{\frac{\lambda}{c}}\right) \varepsilon & \cdots & \left(-\varphi_{1} \sqrt[2 N]{\frac{\lambda}{c}}\right)^{N-1} \varepsilon^{N-1} & \mathrm{e}^{-\varphi_{1} \sqrt[2 N]{\lambda / c}(b-a)} \\
\vdots & \left(-\varphi_{2 N} \sqrt[2 N]{\frac{\lambda}{c}}\right) \varepsilon & \cdots & \left(-\varphi_{2 N} \sqrt[2 N]{\frac{\lambda}{c}}\right)^{N-1} \varepsilon^{N-1} & \mathrm{e}^{-\varphi_{2 N} \sqrt[2 N]{\lambda / c}(b-a)}
\end{array}
\end{aligned}
$$

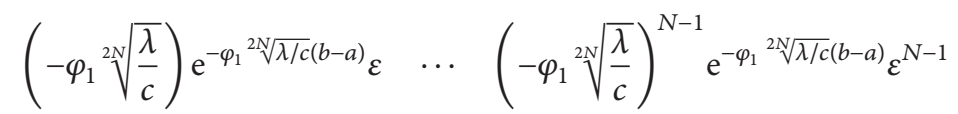

$$
\begin{aligned}
& \left(-\varphi_{2 N} \sqrt[2 N]{\frac{\lambda}{c}}\right) \mathrm{e}^{-\varphi_{2 N} \sqrt[2 N]{\lambda / c}(b-a)} \varepsilon \cdots\left(-\varphi_{2 N} \sqrt[2 N]{\frac{\lambda}{c}}\right)^{N-1} \mathrm{e}^{-\varphi_{2 N} \sqrt[2 N]{\lambda / c}(b-a)} \varepsilon^{N-1} \\
& =\left(\frac{\lambda}{c}\right)^{(N-1) / 2} \varepsilon^{N(N-1)} \mathbf{D}(\lambda) \text {. }
\end{aligned}
$$

Similarly, by using the elementary asymptotics $\mathrm{e}^{j \mu \varepsilon}-1 \underset{\varepsilon \rightarrow 0^{+}}{\sim}$ $i \mu \varepsilon$ and $\mathrm{e}^{i \mu \varepsilon\left(b_{\varepsilon}-a_{\varepsilon}+N-1\right)} \underset{\varepsilon \rightarrow 0^{+}}{\rightarrow} \mathrm{e}^{i \mu(b-a)}$, we obtain that

$$
\begin{aligned}
& \mathrm{e}^{i \mu a} D_{k}\left(\mathrm{e}^{-\lambda \varepsilon^{2 N}}, \mathrm{e}^{i \mu \varepsilon}\right) \\
& \quad=\mathrm{e}^{i \mu a} U\left(u_{1}(\lambda, \varepsilon), \ldots, u_{k-1}(\lambda, \varepsilon),\right.
\end{aligned}
$$

$$
\begin{array}{r}
\left.\mathrm{e}^{i \mu \varepsilon}, u_{k+1}(\lambda, \varepsilon), \ldots, u_{2 N}(\lambda, \varepsilon)\right) \\
\underset{\varepsilon \rightarrow 0^{+}}{\sim}\left(\frac{\lambda}{c}\right)^{(N-1) / 2} \varepsilon^{N(N-1)} \mathbf{D}_{k}(\lambda, \mu),
\end{array}
$$

where $\mathbf{D}_{k}(\lambda, \mu)$ denotes the determinant

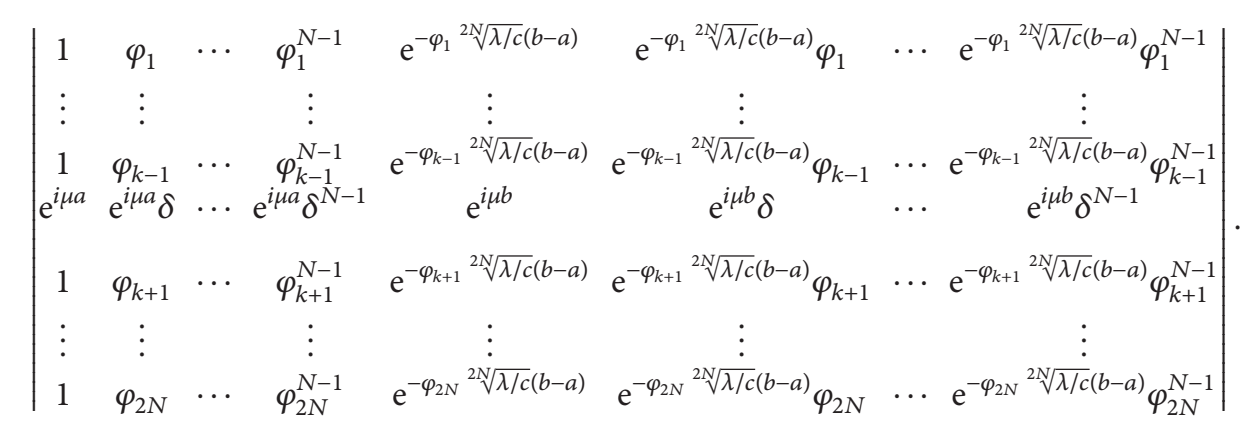

By putting (358) and (359) into (355), we derive that

$$
\mathbb{E}\left(\mathrm{e}^{-\lambda \tau_{a b}+i \mu X_{a b}} \mathbb{1}_{\left\{\tau_{a b}<+\infty\right\}}\right)=\sum_{k=1}^{2 N} \frac{\mathbf{D}_{k}(\lambda, \mu)}{\mathbf{D}(\lambda)} .
$$

It is plain that $\mathbf{D}_{k}(\lambda, \mu)=\mathrm{e}^{i \mu a} \mathbf{D}_{k}^{-}(\lambda, \mu)+\mathrm{e}^{i \mu b} \mathbf{D}_{k}^{-}(\lambda, \mu)$ which finishes the proof of Theorem 57.

Theorem 58. The following convergence holds:

$$
X_{a b}^{\varepsilon} \underset{\varepsilon \rightarrow 0^{+}}{\longrightarrow} X_{a b}
$$

where, for any $\mu \in \mathbb{R}$,

$$
\mathbb{E}\left(\mathrm{e}^{i \mu X_{a b}} \mathbb{1}_{\left\{\tau_{a b}<+\infty\right\}}\right)=\mathrm{e}^{i \mu a} \sum_{j=0}^{N-1} \mathbf{I}_{a b, j}^{-}(i \mu)^{j}+\mathrm{e}^{i \mu b} \sum_{j=0}^{N-1} \mathbf{I}_{a b, j}^{+}(i \mu)^{j}
$$

with

$$
\begin{aligned}
& \mathbf{I}_{a b, j}^{-}=\left(\frac{b}{b-a}\right)^{N} \frac{(-a)^{j}}{j !} \sum_{k=0}^{N-j-1}\left(\begin{array}{c}
k+N-1 \\
k
\end{array}\right)\left(\frac{-a}{b-a}\right)^{k}, \\
& \mathbf{I}_{a b, j}^{+}=\left(\frac{-a}{b-a}\right)^{N} \frac{(-b)^{j}}{j !} \sum_{k=0}^{N-j-1}\left(\begin{array}{c}
k+N-1 \\
k
\end{array}\right)\left(\frac{b}{b-a}\right)^{k} .
\end{aligned}
$$


Moreover,

$$
\mathbb{P}\left\{\tau_{a b}<+\infty\right\}=1 .
$$

Proof. By Definition 34 and by (331),

$$
\begin{aligned}
\mathbb{E}\left(\mathrm { e } ^ { i \mu X _ { a b } \mathbb { 1 } _ { \{ \tau _ { a b } < + \infty \} } ) = } \operatorname { l i m } _ { \varepsilon \rightarrow 0 ^ { + } } \mathbb { E } \left(\mathrm{e}^{\left.i \mu X_{a b}^{\varepsilon} \mathbb{1}_{\left\{\tau_{a b}^{\varepsilon}<+\infty\right\}}\right)}\right.\right. \\
=\lim _{\varepsilon \rightarrow 0^{+}} \mathbb{E}\left(\mathrm{e}^{i \mu \varepsilon S_{a_{\varepsilon}, b_{\varepsilon}}} \mathbb{1}_{\left\{\sigma_{\left.a_{\varepsilon}, b_{\varepsilon}<+\infty\right\}}\right)}\right. \\
=\lim _{\varepsilon \rightarrow 0^{+}}\left(\mathrm{e}^{i \mu \varepsilon a_{\varepsilon}} \sum_{j=0}^{N-1} I_{a_{\varepsilon}, b_{\varepsilon}, j}\left(1-\mathrm{e}^{-i \mu \varepsilon}\right)^{j}\right. \\
\left.+\mathrm{e}^{i \mu \varepsilon b_{\varepsilon}} \sum_{j=0}^{N-1} I_{a_{\varepsilon}, b_{\varepsilon}, j}^{+}\left(\mathrm{e}^{i \mu \varepsilon}-1\right)^{j}\right) \\
=\mathrm{e}^{i \mu a} \lim _{\varepsilon \rightarrow 0^{+}} \sum_{j=0}^{N-1} I_{a_{\varepsilon}, b_{\varepsilon}, j}^{-}\left(1-\mathrm{e}^{-i \mu \varepsilon}\right)^{j} \\
+\mathrm{e}^{i \mu b} \lim _{\varepsilon \rightarrow 0^{+}} \sum_{j=0}^{N-1} I_{a_{\varepsilon}, b_{\varepsilon}, j}^{+}\left(\mathrm{e}^{i \mu \varepsilon}-1\right)^{j}
\end{aligned}
$$

(366)

Concerning, for example, the quantity $I_{a_{\varepsilon}, b_{\varepsilon}, j}^{+}$, we have that

$$
\begin{aligned}
& I_{a_{\varepsilon}, b_{\varepsilon}, j}^{+} \\
& =\frac{(-1)^{j}}{(N-1) !^{2}}\left(\begin{array}{c}
N-1 \\
j
\end{array}\right) \prod_{k=0}^{N-1}\left[\left(k-a_{\varepsilon}\right)\left(k+b_{\varepsilon}\right)\right] \\
& \quad \times \iint_{D^{+}} u^{-a_{\varepsilon}-1}(1-u)^{N-1} v^{j+b_{\varepsilon}-1}(1-v)^{N-j-1} \mathrm{~d} u \mathrm{~d} v .
\end{aligned}
$$

By performing the change of variables $(u, v)=(x, x y)$ in the above integral and by expanding $(1-x y)^{N-j-1}$ as

$$
\begin{aligned}
(1-x y)^{N-j-1} & \\
& =[(1-x)+x(1-y)]^{N-j-1} \\
& =\sum_{k=0}^{N-j-1}\left(\begin{array}{c}
N-j-1 \\
k
\end{array}\right)(1-x)^{k} x^{N-j-k-1}(1-y)^{N-j-k-1}
\end{aligned}
$$

we get that

$$
\begin{gathered}
\iint_{\mathscr{D}^{+}} u^{-a_{\varepsilon}-1}(1-u)^{N-1} v^{j+b_{\varepsilon}-1}(1-v)^{N-j-1} \mathrm{~d} u \mathrm{~d} v \\
=\int_{0}^{1} \int_{0}^{1} x^{j+b_{\varepsilon}-a_{\varepsilon}-1}(1-x)^{N-1} y^{j+b_{\varepsilon}-1} \\
\times(1-x y)^{N-j-1} \mathrm{~d} x \mathrm{~d} y
\end{gathered}
$$

$$
\begin{aligned}
&= \sum_{k=0}^{N-j-1}\left(\begin{array}{c}
N-j-1 \\
k
\end{array}\right) \\
& \times \int_{0}^{1} x^{b_{\varepsilon}-a_{\varepsilon}+N-k-2}(1-x)^{k+N-1} \mathrm{~d} x \\
& \times \int_{0}^{1} y^{j+b_{\varepsilon}-1}(1-y)^{N-j-k-1} \mathrm{~d} y \\
&= \sum_{k=0}^{N-j-1}\left(\begin{array}{c}
N-j-1 \\
k
\end{array}\right) \\
& \times \frac{\left(b_{\varepsilon}-a_{\varepsilon}+N-k-2\right) !(k+N-1) !}{\left(b_{\varepsilon}-a_{\varepsilon}+2 N-2\right) !} \\
& \times \frac{\left(j+b_{\varepsilon}-1\right) !(N-j-k-1) !}{\left(b_{\varepsilon}+N-k-1\right) !} \\
&= \frac{(N-1) !(N-j-1) !\left(j+b_{\varepsilon}-1\right) !}{\left(b_{\varepsilon}-a_{\varepsilon}+2 N-2\right) !} \\
& \times \sum_{k=0}^{N-j-1}\left(\begin{array}{c}
k+N-1 \\
k
\end{array}\right) \frac{\left(b_{\varepsilon}-a_{\varepsilon}+N-k-2\right) !}{\left(b_{\varepsilon}+N-k-1\right) !} .
\end{aligned}
$$

By putting the asymptotics

$$
\begin{aligned}
& \frac{\left(j+b_{\varepsilon}-1\right) !}{\left(b_{\varepsilon}+N-k-1\right) !} \\
& \quad=\frac{1}{\left(b_{\varepsilon}+N-k-1\right)_{N-j-k}} \underset{\varepsilon \rightarrow 0^{+}}{\sim}\left(\frac{\varepsilon}{b}\right)^{N-j-k}, \\
& \frac{\left(b_{\varepsilon}-a_{\varepsilon}+N-k-2\right) !}{\left(b_{\varepsilon}-a_{\varepsilon}+2 N-2\right) !} \\
& =\frac{1}{\left(b_{\varepsilon}-a_{\varepsilon}+2 N-2\right)_{N+k}} \underset{\varepsilon \rightarrow 0^{+}}{\sim}\left(\frac{\varepsilon}{b-a}\right)^{N+k}
\end{aligned}
$$

into (369), we obtain that

$$
\begin{aligned}
\iint_{\mathscr{D}^{+}} u^{-a_{\varepsilon}-1}(1-u)^{N-1} v^{j+b_{\varepsilon}-1}(1-v)^{N-j-1} \mathrm{~d} u \mathrm{~d} v \\
\underset{\varepsilon \rightarrow 0^{+}}{\sim} \frac{(N-1) !(N-j-1) !}{b^{N-j}(b-a)^{N}} \\
\quad \times \varepsilon^{2 N-j} \sum_{k=0}^{N-j-1}\left(\begin{array}{c}
k+N-1 \\
k
\end{array}\right)\left(\frac{b}{b-a}\right)^{k} .
\end{aligned}
$$

Next, using the asymptotics

$$
\prod_{k=0}^{N-1}\left[\left(k-a_{\varepsilon}\right)\left(k+b_{\varepsilon}\right)\right] \underset{\varepsilon \rightarrow 0^{+}}{\sim}(-1)^{N} \frac{(a b)^{N}}{\varepsilon^{2 N}},
$$


expression (367) admits the following asymptotics:

$$
\begin{aligned}
I_{a_{\varepsilon}, b_{\varepsilon}, j}^{+} \underset{\varepsilon \rightarrow 0^{+}}{\sim} & \frac{(-1)^{j+N} b^{j}}{j ! \varepsilon^{j}}\left(\frac{a}{b-a}\right)^{N} \\
& \times \sum_{k=0}^{N-j-1}\left(\begin{array}{c}
k+N-1 \\
k
\end{array}\right)\left(\frac{b}{b-a}\right)^{k} .
\end{aligned}
$$

Then, we see that the second limit lying in (366) equals

$$
\left(\frac{-a}{b-a}\right)^{N} \sum_{j=0}^{N-1} \frac{(-i \mu b)^{j}}{j !}\left[\sum_{k=0}^{N-j-1}\left(\begin{array}{c}
k+N-1 \\
k
\end{array}\right)\left(\frac{b}{b-a}\right)^{k}\right] .
$$

In the same way, it may be seen that the first term of the sum lying in (366) tends to

$$
\left(\frac{b}{b-a}\right)^{N} \sum_{j=0}^{N-1} \frac{(-i \mu a)^{j}}{j !}\left[\sum_{k=0}^{N-j-1}\left(\begin{array}{c}
k+N-1 \\
k
\end{array}\right)\left(\frac{-a}{b-a}\right)^{k}\right] .
$$

As a result, we derive (363).

Finally, let us have a look on the pseudoprobability $\mathbb{P}\left\{\tau_{a b}<+\infty\right\}$. We have that

$$
\begin{aligned}
& \mathbf{I}_{a b, 0}^{-}=\left(\frac{b}{b-a}\right)^{N} \sum_{k=0}^{N-1}\left(\begin{array}{c}
k+N-1 \\
k
\end{array}\right)\left(\frac{-a}{b-a}\right)^{k} \\
&=\frac{b^{N}}{(b-a)^{2 N-1}} \sum_{k=0}^{N-1}\left(\begin{array}{c}
k+N-1 \\
k
\end{array}\right)(-a)^{k}(b-a)^{N-1-k} \\
&=\frac{b^{N}}{(b-a)^{2 N-1}} \sum_{\substack{0 \leq k \leq N-1 \\
0 \leq \ell \leq N-1-k}}\left(\begin{array}{c}
k+N-1 \\
k
\end{array}\right)\left(\begin{array}{c}
N-1-k \\
\ell
\end{array}\right) \\
&=\frac{1}{(b-a)^{2 N-1}} \sum_{m=0}^{N-1}\left[\sum_{k=0}^{m}\left(\begin{array}{c}
k+N-1 \\
k
\end{array}\right)\left(\begin{array}{c}
N-1-k \\
m-k
\end{array}\right)\right] \\
& \times(-a)^{m} b^{2 N-1-m} .
\end{aligned}
$$

By using the elementary identity $\sum_{k=0}^{n}\left(\begin{array}{c}k+p \\ k\end{array}\right)\left(\begin{array}{c}n+q-k \\ n-k\end{array}\right)=$ $\left(\begin{array}{c}n+p+q+1 \\ n\end{array}\right)$ which comes from the equality $(1+x)^{-p}(1+x)^{-q}=$ $(1+x)^{-p-q}$ together with the expansion, for example, for $p$, $(1+x)^{-p}=\sum_{k=0}^{\infty}(-1)^{k}\left(\begin{array}{c}k+p-1 \\ k\end{array}\right) x^{k}$, we get that

$$
\sum_{k=0}^{m}\left(\begin{array}{c}
k+N-1 \\
k
\end{array}\right)\left(\begin{array}{c}
N-1-k \\
m-k
\end{array}\right)=\left(\begin{array}{c}
2 N-1 \\
m
\end{array}\right)
$$

As a byproduct,

$$
\mathbf{I}_{a b, 0}^{-}=\frac{1}{(b-a)^{2 N-1}} \sum_{m=0}^{N-1}\left(\begin{array}{c}
2 N-1 \\
m
\end{array}\right)(-a)^{m} b^{2 N-1-m} .
$$

Similarly,

$$
\mathbf{I}_{a b, 0}^{+}=\frac{1}{(b-a)^{2 N-1}} \sum_{m=N}^{2 N-1}\left(\begin{array}{c}
2 N-1 \\
m
\end{array}\right)(-a)^{m} b^{2 N-1-m}
$$

and we deduce that

$$
\begin{aligned}
\mathbb{P}\left\{\tau_{a b}<+\infty\right\} & =\mathbf{I}_{a b, 0}^{-}+\mathbf{I}_{a b, 0}^{+} \\
& =\frac{1}{(b-a)^{2 N-1}} \sum_{m=0}^{2 N-1}\left(\begin{array}{c}
2 N-1 \\
m
\end{array}\right)(-a)^{m} b^{2 N-1-m} \\
& =1 .
\end{aligned}
$$

The proof of Theorem 58 is finished.

Corollary 59. The pseudodensity of $X_{a b}$ is given by

$$
\frac{\mathbb{P}\left\{X_{a b} \in \mathrm{d} z\right\}}{\mathrm{d} z}=\sum_{j=0}^{N-1}(-1)^{j} \mathbf{I}_{a b, j}^{-} \delta_{a}^{(j)}(z)+\sum_{j=0}^{N-1}(-1)^{j} \mathbf{I}_{a b, j}^{+} \delta_{b}^{(j)}(z) .
$$

In particular,

$$
\mathbb{P}\left\{\tau_{a}^{-}<\tau_{b}^{+}\right\}=\mathbf{I}_{a b, 0}^{-}, \quad \mathbb{P}\left\{\tau_{b}^{+}<\tau_{a}^{-}\right\}=\mathbf{I}_{a b, 0}^{+} .
$$

This result has been announced in [13] without any proof. We will develop it in a forthcoming paper.

\section{Appendix}

\section{A.}

A.1. Lacunary Vandermonde Systems. Let us introduce the "lacunary" Vandermonde determinant (of type $(p+r) \times(p+$ $r)$ ):

$$
\begin{aligned}
U_{p q r} & \left(u_{1}, \ldots, u_{p+r}\right) \\
& =\left|\begin{array}{ccccccc}
1 & u_{1} & \cdots & u_{1}^{p-1} & u_{1}^{p+q} & \cdots & u_{1}^{p+q+r-1} \\
\vdots & \vdots & & \vdots & \vdots & & \vdots \\
1 & u_{p+r} & \cdots & u_{p+r}^{p-1} & u_{p+r}^{p+q} & \cdots & u_{p+r}^{p+q+r-1}
\end{array}\right| .
\end{aligned}
$$

We put $s_{0}:=s_{0}\left(u_{1}, \ldots, u_{p+r}\right)=1$ and, for $\ell \in\{1, \ldots, p+r\}$,

$$
s_{\ell}:=s_{\ell}\left(u_{1}, \ldots, u_{p+r}\right)=\sum_{1 \leq i_{1}<\cdots<i_{\ell} \leq p+r} u_{i_{1}} \cdots u_{i_{\ell}}
$$

We say "lacunary" because it comes from a genuine Vandermonde determinant where the powers from $p$ to $(p+q-1)$ are 
missing. More precisely, the determinant $U_{p q r}\left(u_{1}, \ldots, u_{p+r}\right)$ is extracted from the classical Vandermonde determinant $V_{p+q+r}\left(u_{1}, \ldots, u_{p+q+r}\right)$ of type $(p+q+r) \times(p+q+r)$ by removing the $q$ last rows and the $(p+1)$ st, $(p+2)$ nd, ..., $(p+$ $q)$ th columns. We decompose $V_{p+q+r}\left(u_{1}, \ldots, u_{p+q+r}\right)$ into blocks as follows:

$$
\left|\begin{array}{cccc|ccc|ccc}
1 & u_{1} & \cdots & u_{1}^{p-1} & u_{1}^{p} & \cdots & u_{1}^{p+q-1} & u_{1}^{p+q} & \cdots & u_{1}^{p+q+r-1} \\
\vdots & \vdots & & \vdots & \vdots & & \vdots & \vdots & & \vdots \\
1 & u_{p+r} & \cdots & u_{p+r}^{p-1} & u_{p+r}^{p} & \cdots & u_{p+r}^{p+q-1} & u_{p+r}^{p+q} & \cdots & u_{p+r}^{p+q+r-1} \\
\hline 1 & u_{p+r+1} & \cdots & u_{p+r+1}^{p-1} & u_{p+r+1}^{p} & \cdots & u_{p+r+1}^{p+q-1} & u_{p+r+1}^{p+q} & \cdots & u_{p+r+1}^{p+q+r-1} \\
\vdots & \vdots & & \vdots & \vdots & & \vdots & \vdots & & \vdots \\
1 & u_{p+q+r} & \cdots & u_{p+q+r}^{p-1} & u_{p+q+r}^{p} & \cdots & u_{p+q+r}^{p+q-1} & u_{p+q+r}^{p+q} & \cdots & u_{p+q+r}^{p+q+r-1}
\end{array}\right| .
$$

By moving the $r$ last columns before the $q$ previous ones, this determinant can be rewritten as

$$
(-1)^{q r}\left|\begin{array}{ccccccc|ccc}
1 & u_{1} & \cdots & u_{1}^{p-1} & u_{1}^{p+q} & \cdots & u_{1}^{p+q+r-1} & u_{1}^{p} & \cdots & u_{1}^{p+q-1} \\
\vdots & \vdots & & \vdots & \vdots & & \vdots & \vdots & & \vdots \\
1 & u_{p+r} & \cdots & u_{p+r}^{p-1} & u_{p+r}^{p+q} & \cdots & u_{p+r}^{p+q+r-1} & u_{p+r}^{p} & \cdots & u_{p+r}^{p+q-1} \\
\hline 1 & u_{p+r+1} & \cdots & u_{p+r+1}^{p-1} & u_{p+r+1}^{p+q} & \cdots & u_{p+r+1}^{p+q+r-1} & u_{p+r+1}^{p} & \cdots & u_{p+r+1}^{p+q-1} \\
\vdots & \vdots & & \vdots & \vdots & & \vdots & \vdots & & \vdots \\
1 & u_{p+q+r} & \cdots & u_{p+q+r}^{p-1} & u_{p+q+r}^{p+q} & \cdots & u_{p+q+r}^{p+q+r-1} & u_{p+q+r}^{p} & \cdots & u_{p+q+r}^{p+q-1}
\end{array}\right| .
$$

By appealing to an expansion by blocks of a determinant, it may be seen that $U_{p q r}\left(u_{1}, \ldots, u_{p+r}\right)$ is the cofactor of the "south-east" block of the above determinant. Since the product of, for example, the diagonal terms of this last block is $u_{p+r+1}^{p} u_{p+r+2}^{p+1} \cdots u_{p+q+r}^{p+q-1}$, the determinant $U_{p q r}\left(u_{1}, \ldots, u_{p+r}\right)$ is also the coefficient of $u_{p+r+1}^{p} u_{p+r+2}^{p+1} \cdots u_{p+q+r}^{p+q-1}$ in $V_{p+q+r}\left(u_{1}, \ldots, u_{p+q+r}\right)$. Now, let us expand $V_{p+q+r}\left(u_{1}, \ldots, u_{p+q+r}\right)$ :

$$
V_{p+q+r}\left(u_{1}, \ldots, u_{p+q+r}\right)=\prod_{1 \leq i<j \leq p+q+r}\left(u_{j}-u_{i}\right)=\prod_{1} \prod_{2} \prod_{3}
$$

with

$$
\begin{aligned}
& \prod_{1}=\prod_{2}\left(u_{j}-u_{i}\right), \\
& \prod_{1 \leq i<j \leq p+r}=\prod_{\substack{1 \leq i \leq p+r \\
p+r+1 \leq j \leq p+q+r}}\left(u_{j}-u_{i}\right), \\
& \prod_{3}=\prod_{p+r+1 \leq i<j \leq p+q+r}\left(u_{j}-u_{i}\right) .
\end{aligned}
$$

We have that

$$
\begin{aligned}
\prod_{2} & =\prod_{j=p+r+1}^{p+q+r}\left[\left(u_{j}-u_{1}\right) \cdots\left(u_{j}-u_{p+r}\right)\right] \\
& =\prod_{j=p+r+1}^{p+q+r} \sum_{k=0}^{p+r}(-1)^{p+r-k} s_{p+r-k} u_{j}^{k}
\end{aligned}
$$

$$
\begin{aligned}
= & \sum_{0 \leq k_{1}, \ldots, k_{q} \leq p+r}(-1)^{q(p+r)-k_{1}-\cdots-k_{q}} \\
& \quad \times s_{p+r-k_{1}} \cdots s_{p+r-k_{q}} u_{p+r+1}^{k_{1}} \cdots u_{p+q+r}^{k_{q}}, \\
\prod_{3}= & V_{q}\left(u_{p+r+1}, \ldots, u_{p+q+r}\right) \\
= & \sum_{\varsigma \in \mathbb{S}_{q}} \epsilon(\varsigma) u_{p+r+1}^{\varsigma(1)-1} \cdots u_{p+q+r}^{\varsigma(q)-1} \\
= & \sum_{\substack{0 \leq \ell_{1}, \ldots, \ell_{q} \leq q-1 \\
\ell_{1}, \ldots, \ell_{q} \text { all different }}} \epsilon\left(\ell_{1} \ldots, \ell_{q}\right) u_{p+r+1}^{\ell_{1}} \cdots u_{p+q+r}^{\ell_{q} .}
\end{aligned}
$$

The symbol $\mathfrak{S}_{q}$ in the above sum denotes the set of the permutations of the numbers $1,2, \ldots, q, \epsilon(\varsigma)$ is the signature of the permutation $\varsigma$ and $\epsilon\left(\ell_{1} \ldots, \ell_{q}\right) \in\{-1,+1\}$ is the signature of the permutation mapping $1, \ldots, q$ into $\ell_{1} \ldots, \ell_{q}$. The product $\prod_{2} \prod_{3}$ is given by

$$
\begin{aligned}
(-1)^{q(p+1)} \sum_{\substack{0 \leq k_{1}, \ldots, k_{q} \leq p+r \\
0 \leq \ell_{1}, \ldots, \ell_{q} \leq q-1 \\
\ell_{1}, \ldots, \ell_{q} \text { all different }}}(-1)^{k_{1}+\cdots+k_{q}} \epsilon\left(\ell_{1} \ldots, \ell_{q}\right) \\
\quad \times s_{p+r-k_{1}} \cdots s_{p+r-k_{q}} u_{p+r+1}^{k_{1}+\ell_{1}} \cdots u_{p+q+r}^{k_{q}+\ell_{q}} .
\end{aligned}
$$

For obtaining the coefficient of $u_{p+r+1}^{p} u_{p+r+2}^{p+1} \cdots u_{p+q+r}^{p+q-1}$, we only keep in the foregoing sum the indices $k_{1}, \ldots, k_{q}$, 
$\ell_{1}, \ldots, \ell_{q}$ such that $k_{1}+\ell_{1}=p, k_{2}+\ell_{2}=p+1, \ldots, k_{q}+\ell_{q}=$ $p+q-1$ and $0 \leq k_{1}, \ldots, k_{q} \leq p+r, 0 \leq \ell_{1}, \ldots, \ell_{q} \leq q-1$, the indices $\ell_{1}, \ldots, \ell_{q}$ being all distinct. This gives that

$$
\begin{aligned}
\prod_{2} \prod_{3}=\sum_{\begin{array}{r}
\text { for } i \in\{1, \ldots, q\}: \\
(i-r-1) \vee 0 \leq \ell_{\ell} \leq(p+i-1) \wedge(q-1) \\
\ell_{1}, \ldots, \ell_{q} \text { all different }
\end{array}} & \epsilon\left(\ell_{1} \ldots, \ell_{q}\right) \\
& \times s_{\ell_{1}+r} s_{\ell_{2}+r-1} \cdots s_{\ell_{q}+r-q+1} .
\end{aligned}
$$

Finally, we can observe that the foregoing sum is nothing but the expansion of the determinant

$$
\mathcal{S}_{p q r}=\left|\begin{array}{cccc}
s_{r} & s_{r-1} & \cdots & s_{r-q+1} \\
s_{r+1} & s_{r} & \cdots & s_{r-q+2} \\
\vdots & \vdots & & \vdots \\
s_{r+q-1} & s_{r+q-2} & \cdots & s_{r}
\end{array}\right|
$$

As a result, we obtain the result below.

Proposition A.1. The determinant $U_{p q r}\left(u_{1}, \ldots, u_{p+r}\right)$ admits the following expression:

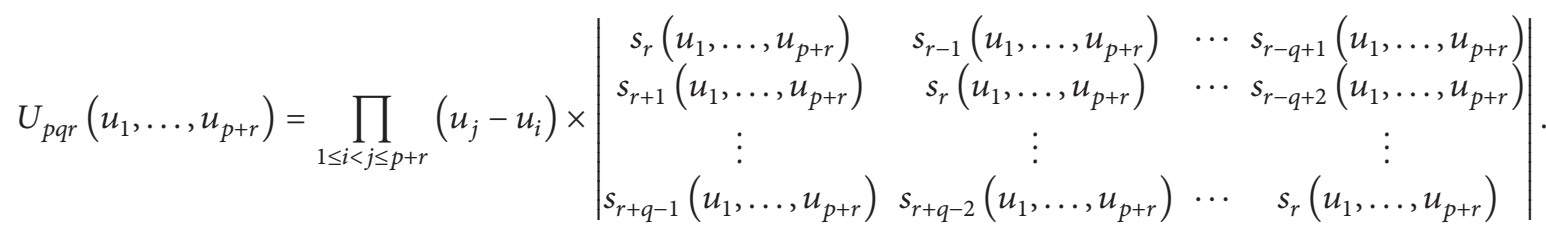

Let now $U_{p q r, \ell}\left(\begin{array}{l}u_{1}, \ldots, u_{p+r} \\ \alpha_{1}, \ldots, \alpha_{p+r}\end{array}\right)$ be the determinant deduced from $U_{p q r}\left(u_{1}, \ldots, u_{p+r}\right)$ by replacing one column by a general column $\alpha_{1}, \ldots, \alpha_{p+r}$, that is, the determinant given, if $0 \leq \ell \leq$ $p-1$, by

$$
\left|\begin{array}{ccccccccccc}
1 & u_{1} & \cdots & u_{1}^{\ell-1} & \alpha_{1} & u_{1}^{\ell+1} & \cdots & u_{1}^{p-1} & u_{1}^{p+q} & \cdots & u_{1}^{p+q+r-1} \\
\vdots & \vdots & & \vdots & \vdots & \vdots & & \vdots & \vdots & & \vdots \\
1 & u_{p+r} & \cdots & u_{p+r}^{\ell-1} & \alpha_{p+r} & u_{p+r}^{\ell+1} & \cdots & u_{p+r}^{p-1} & u_{p+r}^{p+q} & \cdots & u_{p+r}^{p+q+r-1}
\end{array}\right|,
$$

and, if $p+q \leq \ell \leq p+q+r-1$, by

$$
\left|\begin{array}{ccccccccccc}
1 & u_{1} & \cdots & u_{1}^{p-1} & u_{1}^{p+q} & \cdots & u_{1}^{\ell-1} & \alpha_{1} & u_{1}^{\ell+1} & \cdots & u_{1}^{p+q+r-1} \\
\vdots & \vdots & & \vdots & \vdots & & \vdots & \vdots & \vdots & & \vdots \\
1 & u_{p+r} & \cdots & u_{p+r}^{p-1} & u_{p+r}^{p+q} & \cdots & u_{p+r}^{\ell-1} & \alpha_{p+r} & u_{p+r}^{\ell+1} & \cdots & u_{p+r}^{p+q+r-1}
\end{array}\right| .
$$

We have that

$$
\begin{aligned}
U_{p q r, \ell} & \left(\begin{array}{l}
u_{1}, \ldots, u_{p+r} \\
\alpha_{1}, \ldots, \alpha_{p+r}
\end{array}\right) \\
= & \sum_{k=1}^{p+r} \alpha_{k} U_{p q r, k \ell}\left(u_{1}, \ldots, u_{k-1}, u_{k+1}, \ldots, u_{p+r}\right),
\end{aligned}
$$

where $U_{p q r, k \ell}\left(u_{1}, \ldots, u_{k-1}, u_{k+1}, \ldots, u_{p+r}\right)$ is the determinant given, if $0 \leq \ell \leq p-1$, by

$$
\left|\begin{array}{ccccccccccc}
1 & u_{1} & \cdots & u_{1}^{\ell-1} & 0 & u_{1}^{\ell+1} & \cdots & u_{1}^{p-1} & u_{1}^{p+q} & \cdots & u_{1}^{p+q+r-1} \\
\vdots & \vdots & & \vdots & \vdots & \vdots & & \vdots & \vdots & & \vdots \\
1 & u_{k-1} & \cdots & u_{k-1}^{\ell-1} & 0 & u_{k-1}^{\ell+1} & \cdots & u_{k-1}^{p-1} & u_{k-1}^{p+q} & \cdots & u_{k-1}^{p+q+r-1} \\
1 & u_{k} & \cdots & u_{k}^{\ell-1} & 1 & u_{k}^{\ell+1} & \cdots & u_{k}^{p-1} & u_{k}^{p+q} & \cdots & u_{k}^{p+q+r-1} \\
1 & u_{k+1} & \cdots & u_{k+1}^{\ell-1} & 0 & u_{k+1}^{\ell+1} & \cdots & u_{k+1}^{p-1} & u_{k+1}^{p+q} & \cdots & u_{k+1}^{p+q+r-1} \\
\vdots & \vdots & & \vdots & \vdots & \vdots & & \vdots & \vdots & & \vdots \\
1 & u_{p+r} & \cdots & u_{p+r}^{\ell-1} & 0 & u_{p+r}^{\ell+1} & \cdots & u_{p+r}^{p-1} & u_{p+r}^{p+q} & \cdots & u_{p+r}^{p+q+r-1}
\end{array}\right|
$$

and, if $p+q \leq \ell \leq p+q+r-1$, by

$$
\left|\begin{array}{ccccccccccc}
1 & u_{1} & \cdots & u_{1}^{p-1} & u_{1}^{p+q} & \cdots & u_{1}^{\ell-1} & 0 & u_{1}^{\ell+1} & \cdots & u_{1}^{p+q+r-1} \\
\vdots & \vdots & & \vdots & \vdots & & \vdots & \vdots & \vdots & & \vdots \\
1 & u_{k-1} & \cdots & u_{k-1}^{p-1} & u_{k-1}^{p+q} & \cdots & u_{k-1}^{\ell-1} & 0 & u_{k-1}^{\ell+1} & \cdots & u_{k-1}^{p+q+r-1} \\
1 & u_{k} & \cdots & u_{k}^{p-1} & u_{k}^{p+q} & \cdots & u_{k}^{\ell-1} & 1 & u_{k}^{\ell+1} & \cdots & u_{k}^{p+q+r-1} \\
1 & u_{k+1} & \cdots & u_{k+1}^{p-1} & u_{k+1}^{p+q} & \cdots & u_{k+1}^{\ell-1} & 0 & u_{k+1}^{\ell+1} & \cdots & u_{k+1}^{p+q+r-1} \\
\vdots & \vdots & & \vdots & \vdots & & \vdots & \vdots & \vdots & & \vdots \\
1 & u_{p+r} & \cdots & u_{p+r}^{p-1} & u_{p+r}^{p+q} & \cdots & u_{p+r}^{\ell-1} & 0 & u_{p+r}^{\ell+1} & \cdots & u_{p+r}^{p+q+r-1}
\end{array}\right| .
$$

In fact, $U_{p q r, k \ell}\left(u_{1}, \ldots, u_{k-1}, u_{k+1}, \ldots, u_{p+r}\right)$ is the coefficient of $u_{k}^{\ell}$ in $U_{p q r}\left(u_{1}, \ldots, u_{p+r}\right)$. Let us introduce $s_{k, 0}:=s_{k, 0}\left(u_{1}, \ldots\right.$, $\left.u_{k-1}, u_{k+1}, \ldots, u_{p+r}\right)=1, s_{k, m}:=s_{k, m}\left(u_{1}, \ldots, u_{k-1}, u_{k+1}, \ldots\right.$, $\left.u_{p+r}\right)=0$ for any integer $m$ such that $m \leq-1$ or $m \geq p+r$ and, for $m \in\{1, \ldots, p+r-1\}$,

$$
\begin{aligned}
s_{k, m} & :=s_{k, m}\left(u_{1}, \ldots, u_{k-1}, u_{k+1}, \ldots, u_{p+r}\right) \\
& =\sum_{\substack{1 \leq i_{1}<\cdots<i_{m} \leq p+r \\
i_{1}, \ldots, i_{m} \neq k}} u_{i_{1}} \cdots u_{i_{m}} .
\end{aligned}
$$

We need to isolate $u_{k}$ in $\prod_{1 \leq i<j \leq p+r}\left(u_{j}-u_{i}\right)$ and $\mathcal{S}_{p q r}$. First, we write that

$$
\begin{aligned}
& \prod_{1 \leq i<j \leq p+r}\left(u_{j}-u_{i}\right) \\
& =(-1)^{p+r-k} \prod_{\substack{1 \leq i<j \leq p+r \\
i, j \neq k}}\left(u_{j}-u_{i}\right) \times \prod_{\substack{1 \leq i \leq p+r \\
j \neq k}}\left(u_{k}-u_{i}\right) \\
& =(-1)^{k-1} \prod_{\substack{1 \leq i<j \leq p+r \\
i, j \neq k}}\left(u_{j}-u_{i}\right) \sum_{m=0}^{p+r-1}(-1)^{m} s_{k, p+r-m-1} u_{k}^{m} .
\end{aligned}
$$


Second, by isolating $u_{k}$ in $s_{m}$ according to $s_{m}=s_{k, m}+$ $u_{k} s_{k, m-1}$, we get that the determinant $\mathcal{S}_{p q r}$ can be rewritten as

$$
\left|\begin{array}{cccc}
s_{k, r}+u_{k} s_{k, r-1} & s_{k, r-1}+u_{k} s_{k, r-2} & \cdots & s_{k, r-q+1}+u_{k} s_{k, r-q} \\
s_{k, r+1}+u_{k} s_{k, r} & s_{k, r}+u_{k} s_{k, r-1} & \cdots & s_{k, r-q+2}+u_{k} s_{k, r-q+1} \\
\vdots & \vdots & & \vdots \\
s_{k, r+q-1}+u_{k} s_{k, r+q-2} & s_{k, r+q-2}+u_{k} s_{k, r+q-3} & \cdots & s_{k, r}+u_{k} s_{k, r-1}
\end{array}\right| .
$$

By introducing vectors $\vec{V}_{m}$ with coordinates $\left(s_{k, m}, s_{k, m+1}, \ldots\right.$, $s_{k, m+q-1}$ ) (written as a column), this determinant can be rewritten as

$\mathcal{S}_{p q r}=\operatorname{det}\left(\vec{V}_{r}+u_{k} \vec{V}_{r-1}, \vec{V}_{r-1}+u_{k} \vec{V}_{r-2}, \ldots, \vec{V}_{r-q+1}+u_{k} \vec{V}_{r-q}\right)$.

By appealing to multilinearity, it is easy to see that

$$
\delta_{p q r}=\sum_{n=0}^{q} \operatorname{det}\left(\vec{V}_{r}, \vec{V}_{r-1}, \ldots, \vec{V}_{r-q+n+1}, \vec{V}_{r-q+n-1}, \ldots, \vec{V}_{r-q}\right) u_{k}^{n}
$$

Now, let us multiply the sum lying in (A.18) by (A.21):

$$
\begin{aligned}
& \left(\sum_{m=0}^{p+r-1}(-1)^{m} s_{k, p+r-m-1} u_{k}^{m}\right) \\
& \quad \times\left(\sum_{n=0}^{q} \operatorname{det}\left(\vec{V}_{r}, \vec{V}_{r-1}, \ldots, \vec{V}_{r-q+n+1}, \vec{V}_{r-q+n-1}, \ldots, \vec{V}_{r-q}\right) u_{k}^{n}\right)
\end{aligned}
$$

$$
\begin{gathered}
=\sum_{\ell=0}^{p+q+r-1}\left(\sum_{m=0 \vee(\ell-q)}^{(p+r-1) \wedge \ell}(-1)^{m} s_{k, p+r-m-1}\right. \\
\times \operatorname{det}\left(\vec{V}_{r}, \vec{V}_{r-1}, \ldots, \vec{V}_{\ell+r-q-m+1},\right. \\
\left.\left.\vec{V}_{\ell+r-q-m-1}, \ldots, \vec{V}_{r-q}\right)\right) u_{k}^{\ell} .
\end{gathered}
$$

Recalling the convention that $s_{k, m}=0$ if $m \leq-1$ or $m \geq p+r$, the coefficient of $u_{k}^{\ell}$ in (A.22) can be written as

$$
\begin{aligned}
& \sum_{m=\ell-q}^{\ell}(-1)^{m} s_{k, p+r-m-1} \\
& \quad \times \operatorname{det}\left(\vec{V}_{r}, \vec{V}_{r-1}, \ldots, \vec{V}_{\ell+r-q-m+1}, \vec{V}_{\ell+r-q-m-1}, \ldots, \vec{V}_{r-q}\right) \\
& =(-1)^{\ell} \sum_{m=0}^{q}(-1)^{m+q} s_{k, p+q+r-\ell-m-1} \\
& \quad \times \operatorname{det}\left(\vec{V}_{r}, \vec{V}_{r-1}, \ldots, \vec{V}_{r-m+1}, \vec{V}_{r-m-1}, \ldots, \vec{V}_{r-q}\right) .
\end{aligned}
$$

In this form, we see that the coefficient of $u_{k}^{\ell}$ in (A.22) is nothing but the product of $(-1)^{\ell}$ by the expansion of the determinant

$$
\mathcal{S}_{p q r, k \ell}=\left|\begin{array}{cccc}
s_{k, r} & s_{k, r-1} & \cdots & s_{k, r-q} \\
s_{k, r+1} & s_{k, r} & \cdots & s_{k, r-q+1} \\
\vdots & \vdots & & \vdots \\
s_{k, r+q-1} & s_{k, r+q-2} & \cdots & s_{k, r-1} \\
s_{k, p+q+r-\ell-1} & s_{k, p+q+r-\ell-2} & \cdots & s_{k, p+r-\ell-1}
\end{array}\right| .
$$

Proposition A.2. The determinant $U_{p q r, k \ell}\left(u_{1}, \ldots, u_{k-1}\right.$, $\left.u_{k+1}, \ldots, u_{p+r}\right)$ admits the following expression:

$$
\begin{aligned}
U_{p q r, k \ell} & \left(u_{1}, \ldots, u_{k-1}, u_{k+1}, \ldots, u_{p+r}\right) \\
= & (-1)^{k+\ell-1} \prod_{\substack{1 \leq i<j \leq p+r \\
i, j \neq k}}\left(u_{j}-u_{i}\right) \\
& \times\left|\begin{array}{ccc}
s_{k, r}\left(u_{1}, \ldots, u_{k-1}, u_{k+1}, \ldots, u_{p+r}\right) & \ldots & s_{k, r-q}\left(u_{1}, \ldots, u_{k-1}, u_{k+1}, \ldots, u_{p+r}\right) \\
s_{k, r+1}\left(u_{1}, \ldots, u_{k-1}, u_{k+1}, \ldots, u_{p+r}\right) & \cdots & s_{k, r-q+1}\left(u_{1}, \ldots, u_{k-1}, u_{k+1}, \ldots, u_{p+r}\right) \\
\vdots & & \vdots \\
s_{k, r+q-1}\left(u_{1}, \ldots, u_{k-1}, u_{k+1}, \ldots, u_{p+r}\right) & \cdots & s_{k, r-1}\left(u_{1}, \ldots, u_{k-1}, u_{k+1}, \ldots, u_{p+r}\right) \\
s_{k, p+q+r-\ell-1}\left(u_{1}, \ldots, u_{k-1}, u_{k+1}, \ldots, u_{p+r}\right) & \cdots & s_{k, p+r-\ell-1}\left(u_{1}, \ldots, u_{k-1}, u_{k+1}, \ldots, u_{p+r}\right)
\end{array}\right| .
\end{aligned}
$$


As a consequence of Propositions A.1 and A.2, we get the result below. Set

$$
p_{k}:=p_{k}\left(u_{1}, \ldots, u_{p+r}\right)=\prod_{\substack{1 \leq i \leq p+r \\ i \neq k}}\left(u_{k}-u_{i}\right) .
$$

Proposition A.3. Let $p, q, r$ be positive integers, let $u_{1}, \ldots$, $u_{p+r}$ be distinct complex numbers, and let $\alpha_{1}, \ldots, \alpha_{p+r}$ be complex numbers. Set $I=\{0, \ldots, p-1\} \cup\{p+q, \ldots, p+q+r-1\}$. The solution of the system $\sum_{\ell \in I} u_{k}^{\ell} x_{\ell}=\alpha_{k}, 1 \leq k \leq p+r$, or, more explicitly,

$$
\begin{gathered}
x_{0}+u_{1} x_{1}+\cdots+u_{1}^{p-1} x_{p-1}+u_{1}^{p+q} x_{p+q} \\
+\cdots+u_{1}^{p+q+r-1} x_{p+q+r-1}=\alpha_{1} \\
\vdots \\
x_{0}+u_{p+r} x_{1}+\cdots+u_{p+r}^{p-1} x_{p-1}+u_{p+r}^{p+q} x_{p+q} \\
+\cdots+u_{p+r}^{p+q+r-1} x_{p+q+r-1}=\alpha_{p+r}
\end{gathered}
$$

is given by

$$
\begin{aligned}
x_{\ell}= & \frac{(-1)^{\ell+p+r-1}}{\mathcal{S}_{p q r}\left(u_{1}, \ldots, u_{p+r}\right)} \\
& \times \sum_{k=1}^{p+r} \alpha_{k} \frac{\delta_{p q r, k \ell}\left(u_{1}, \ldots, u_{k-1}, u_{k+1}, \ldots, u_{p+r}\right)}{p_{k}\left(u_{1}, \ldots, u_{p+r}\right)},
\end{aligned}
$$

Proof. Cramer's formulae yield that

$$
\begin{aligned}
x_{\ell} & =\frac{U_{p q r, \ell}\left(\begin{array}{c}
u_{1}, \ldots, u_{p+r} \\
\alpha_{1}, \ldots, \alpha_{p+r}
\end{array}\right)}{U_{p q r}\left(u_{1}, \ldots, u_{p+r}\right)} \\
& =\sum_{k=1}^{p+r} \alpha_{k} \frac{U_{p q r, k \ell}\left(u_{1}, \ldots, u_{k-1}, u_{k+1}, \ldots, u_{p+r}\right)}{U_{p q r}\left(u_{1}, \ldots, u_{p+r}\right)} .
\end{aligned}
$$

By using the factorisations provided by Propositions A.1 and A.2, namely,

$$
\begin{aligned}
& U_{p q r}\left(u_{1}, \ldots, u_{p+r}\right) \\
& =V_{p+r}\left(u_{1}, \ldots, u_{p+r}\right) \mathcal{S}_{p q r}\left(u_{1}, \ldots, u_{p+r}\right) \\
& =(-1)^{p+r-k} p_{k}\left(u_{1}, \ldots, u_{p+r}\right) \\
& \quad \times V_{p+r-1}\left(u_{1}, \ldots, u_{k-1}, u_{k+1}, \ldots, u_{p+r}\right) \mathcal{S}_{p q r}\left(u_{1}, \ldots, u_{p+r}\right), \\
& U_{p q r, k \ell}\left(u_{1}, \ldots, u_{k-1}, u_{k+1}, \ldots, u_{p+r}\right) \\
& \quad=(-1)^{k+\ell-1} V_{p+r-1}\left(u_{1}, \ldots, u_{k-1}, u_{k+1}, \ldots, u_{p+r}\right) \\
& \quad \times \mathcal{S}_{p q r, k \ell}\left(u_{1}, \ldots, u_{k-1}, u_{k+1}, \ldots, u_{p+r}\right),
\end{aligned}
$$

we immediately get (A.28).

\section{A.2. A Combinatoric Identity}

Lemma A.4. The following identity holds for any positive integers $\alpha, \beta, n$ :

$$
\sum_{k=0}^{n}(-1)^{k}\left(\begin{array}{l}
n \\
k
\end{array}\right) \frac{(k+\alpha) !}{(k+\beta) !}=\frac{\alpha !}{(\beta+n) !}(\beta-\alpha+n-1)_{n} .
$$

It can be rewritten as

$$
\begin{gathered}
\sum_{k=0}^{n}(-1)^{k}\left(\begin{array}{l}
n \\
k
\end{array}\right)\left(\begin{array}{l}
k+\alpha \\
k+\beta
\end{array}\right)=(-1)^{n}\left(\begin{array}{c}
\alpha \\
\beta+n
\end{array}\right) \quad \text { if } \alpha \geq \beta, \\
\sum_{k=0}^{n} \frac{(-1)^{k}\left(\begin{array}{l}
n \\
k
\end{array}\right)}{\left(\begin{array}{c}
k+\beta \\
k+\alpha
\end{array}\right)}=\frac{(\beta-\alpha) /(\beta-\alpha+n)}{\left(\begin{array}{c}
\beta+n \\
\alpha
\end{array}\right)} \quad \text { if } \alpha<\beta .
\end{gathered}
$$

Proof. Suppose first that $\alpha \geq \beta$. Noticing that

$$
\frac{(k+\alpha) !}{(k+\beta) !}=\left.\frac{\mathrm{d}^{\alpha-\beta}}{\mathrm{d} x^{\alpha-\beta}}\left(x^{k+\alpha}\right)\right|_{x=1},
$$

we immediately get that

$$
\begin{aligned}
\sum_{k=0}^{n}(-1)^{k}\left(\begin{array}{l}
n \\
k
\end{array}\right) \frac{(k+\alpha) !}{(k+\beta) !} & =\left.\frac{\mathrm{d}^{\alpha-\beta}}{\mathrm{d} x^{\alpha-\beta}}\left(\sum_{k=0}^{n}(-1)^{k}\left(\begin{array}{l}
n \\
k
\end{array}\right) x^{k+\alpha}\right)\right|_{x=1} \\
& =\left.\frac{\mathrm{d}^{\alpha-\beta}}{\mathrm{d} x^{\alpha-\beta}}\left(x^{\alpha}(1-x)^{n}\right)\right|_{x=1} .
\end{aligned}
$$

We expand the last displayed derivative by using Leibniz rule:

$$
\begin{aligned}
& \left.\frac{\mathrm{d}^{\alpha-\beta}}{\mathrm{d} x^{\alpha-\beta}}\left(x^{\alpha}(1-x)^{n}\right)\right|_{x=1} \\
& \quad=\left.\left.\sum_{k=0}^{\alpha-\beta}\left(\begin{array}{c}
\alpha-\beta \\
k
\end{array}\right) \frac{\mathrm{d}^{\alpha-\beta-k}}{\mathrm{~d} x^{\alpha-\beta-k}}\left(x^{\alpha}\right)\right|_{x=1} \frac{\mathrm{d}^{k}}{\mathrm{~d} x^{k}}\left((1-x)^{n}\right)\right|_{x=1} .
\end{aligned}
$$

Since $\left.\left(\mathrm{d}^{k} / \mathrm{d} x^{k}\right)\left((1-x)^{n}\right)\right|_{x=1}=(-1)^{n} \delta_{n k} n !$, we have that $\left.\left(\mathrm{d}^{\alpha-\beta} / \mathrm{d} x^{\alpha-\beta}\right)\left(x^{\alpha}(1-x)^{n}\right)\right|_{x=1}=0$ if $\alpha-\beta<n$, and, if $\alpha-\beta \geq n$,

$$
\begin{aligned}
\left.\frac{\mathrm{d}^{\alpha-\beta}}{\mathrm{d} x^{\alpha-\beta}}\left(x^{\alpha}(1-x)^{n}\right)\right|_{x=1} & =\left.(-1)^{n} n !\left(\begin{array}{c}
\alpha-\beta \\
n
\end{array}\right) \frac{\mathrm{d}^{\alpha-\beta-n}}{\mathrm{~d} x^{\alpha-\beta-n}}\left(x^{\alpha}\right)\right|_{x=1} \\
& =(-1)^{n} \frac{\alpha !(\alpha-\beta) !}{(\alpha-\beta-n) !(\beta+n) !}
\end{aligned}
$$

which coincides with the announced result. Second, suppose that $\alpha<\beta$. Noticing that

$$
\frac{(k+\alpha) !}{(k+\beta) !}=\frac{1}{(\beta-\alpha-1) !} \int_{0}^{1} x^{k+\alpha}(1-x)^{\beta-\alpha-1} \mathrm{~d} x,
$$

we get that

$$
\begin{gathered}
\sum_{k=0}^{n}(-1)^{k}\left(\begin{array}{l}
n \\
k
\end{array}\right) \frac{(k+\alpha) !}{(k+\beta) !} \\
=\frac{1}{(\beta-\alpha-1) !}
\end{gathered}
$$




$$
\begin{aligned}
& \times \int_{0}^{1}\left(\sum_{k=0}^{n}(-1)^{k}\left(\begin{array}{l}
n \\
k
\end{array}\right) x^{k}\right) x^{\alpha}(1-x)^{\beta-\alpha-1} \mathrm{~d} x \\
= & \frac{1}{(\beta-\alpha-1) !} \int_{0}^{1} x^{\alpha}(1-x)^{\beta-\alpha+n-1} \mathrm{~d} x \\
= & \frac{\alpha !(\beta-\alpha+n-1) !}{(\beta-\alpha-1) !(\beta+n) !}
\end{aligned}
$$

which coincides with the announced result.

A.3. Some Matrices. Let $\alpha, \beta \in \mathbb{N}$ such that $\alpha>\beta \geq N$ and set

$$
\mathbf{A}=\left[\left(\begin{array}{c}
j+\alpha \\
i+N
\end{array}\right)\right]_{0 \leq i, j \leq N-1}, \quad \mathbf{B}=\left[\left(\begin{array}{c}
\beta \\
i+N
\end{array}\right)\right]_{0 \leq i \leq N-1}
$$

with the convention of settings $\left(\begin{array}{l}i \\ j\end{array}\right)=0$ if $i>j$. These matrices have been used for solving systems (273) and (274) with the choices $\alpha=b-a+N-1$ and $\beta=N-a-1$. The aim of this section is to compute the product of the inverse of $\mathbf{A}$ by $\mathbf{B}$, namely, $\mathbf{A}^{-1} \mathbf{B}$. For this, we use Gauss elimination. The result is displayed in Theorem A.8. The calculations are quite technical, so we perform them progressively, the intermediate steps being stated in several lemmas (Lemmas A.4, A.5, A.6, and A.7).

Lemma A.5. The matrix $\mathbf{A}$ can be decomposed into $\mathbf{A}=\mathbf{L U}^{-1}$, where the matrices $\mathbf{U}$ and $\mathbf{L}$ are given by

$$
\begin{gathered}
\mathbf{U}=\left[(-1)^{i+j} \mathbb{1}_{\{i \leq j\}}\left(\begin{array}{l}
j \\
i
\end{array}\right) \frac{(j+\alpha)_{N}}{(i+\alpha)_{N}}\right]_{0 \leq i, j \leq N-1}, \\
\mathbf{L}=\left[\mathbb{1}_{\{i \geq j\}}\left(\begin{array}{c}
j+\alpha \\
i+N
\end{array}\right) \frac{(i)_{j}}{(j+\alpha-N)_{j}}\right]_{0 \leq i, j \leq N-1} .
\end{gathered}
$$

The regular matrices $\mathbf{U}$ and $\mathbf{L}$, are respectively, upper and lower triangular.
Proof. We begin by detailing the algorithm providing the matrix U. Call $\mathbf{C}_{0}^{(0)}, \mathbf{C}_{1}^{(0)}, \ldots, \mathbf{C}_{N-1}^{(0)}$ the columns of $\mathbf{A}$, that is, for $j \in\{0, \ldots, N-1\}$,

$$
\mathbf{C}_{j}^{(0)}=\left[\left(\begin{array}{c}
j+\alpha \\
i+N
\end{array}\right)\right]_{0 \leq i \leq N-1}
$$

Apply to them, except for $\mathbf{C}_{0}^{(0)}$, the transformation defined, for $j \in\{1, \ldots, N-1\}$, by

$$
\mathbf{C}_{j}^{(1)}=\mathbf{C}_{j}^{(0)}-\frac{j+\alpha}{j+\alpha-N} \mathbf{C}_{j-1}^{(0)} \text {. }
$$

The $\mathbf{C}_{0}^{(0)}, \mathbf{C}_{1}^{(1)}, \ldots, \mathbf{C}_{N-1}^{(1)}$ are the columns of a new matrix $\mathbf{L}_{1}$. Actually, this transformation corresponds to a matrix multiplication acting on $\mathbf{A}: \mathbf{L}_{1}=\mathbf{A U}_{1}$ with

$$
\mathbf{U}_{1}=\left[\begin{array}{c|cccc}
1 & -\frac{\alpha+1}{\alpha+1-N} & 0 & & 0 \\
0 & 1 & -\frac{\alpha+2}{\alpha+2-N} & \vdots \\
0 & 0 & 1 & \ddots & 0 \\
\vdots & \vdots & & \ddots & -\frac{\alpha+N-1}{\alpha-1} \\
0 & 0 & 0 & & 1
\end{array}\right] .
$$

Simple computations show that

$$
\begin{aligned}
\mathbf{L}_{1} & =\left[\left(\delta_{j 0}+\mathbb{1}_{\{j \geq 1\}} \frac{i}{j+\alpha-N}\right)\left(\begin{array}{c}
j+\alpha \\
i+N
\end{array}\right)\right]_{0 \leq i, j \leq N-1} \\
& =\left[\left(\begin{array}{c}
j+\alpha \\
i+N
\end{array}\right) \frac{(i)_{j \wedge 1}}{(j+\alpha-N)_{j \wedge 1}}\right]_{0 \leq i, j \leq N-1} .
\end{aligned}
$$

Next, apply the second transformation to the columns of $\mathbf{L}_{1}$ except for $\mathbf{C}_{0}^{(0)}$ and $\mathbf{C}_{1}^{(1)}$, defined, for $j \in\{2, \ldots, N-1\}$, by

$$
\begin{aligned}
\mathbf{C}_{j}^{(2)}= & \mathbf{C}_{j}^{(1)}-\frac{j+\alpha}{j+\alpha-N} \mathbf{C}_{j-1}^{(1)} \\
= & \mathbf{C}_{j}^{(0)}-2 \frac{j+\alpha}{j+\alpha-N} \mathbf{C}_{j-1}^{(0)} \\
& +\frac{(j+\alpha)(j+\alpha-1)}{(j+\alpha-N)(j+\alpha-N-1)} \mathbf{C}_{j-2}^{(1)} .
\end{aligned}
$$

The $\mathbf{C}_{0}^{(0)}, \mathbf{C}_{1}^{(1)}, \mathbf{C}_{2}^{(2)}, \ldots, \mathbf{C}_{N-1}^{(2)}$ are the columns of a new matrix $\mathbf{L}_{2}=\mathbf{A U}_{2}$, where

$$
\mathbf{U}_{2}=\left[\begin{array}{cc|ccc}
1 & -\frac{\alpha+1}{\alpha+1-N} & \frac{(\alpha+2)(\alpha+1)}{(\alpha+2-N)(\alpha+1-N)} & 0 \\
0 & 1 & -2 \frac{\alpha+2}{\alpha+2-N} & \ddots & \vdots \\
0 & 0 & 1 & \ddots & \frac{(\alpha+N-1)(\alpha+N-2)}{(\alpha-1)(\alpha-2)} \\
\vdots & \vdots & 0 & \ddots & -2 \frac{\alpha+N-1}{\alpha-1} \\
0 & 0 & 0 & & 1
\end{array}\right] .
$$


Straightforward algebra yields that

$$
\begin{aligned}
\mathbf{L}_{2}=[ & \left(\delta_{j 0}+\frac{i}{j+\alpha-N} \delta_{j 1}\right. \\
& \left.+\mathbb{1}_{\{j \geq 2\}} \frac{i(i-1)}{(j+\alpha-N)(j+\alpha-N-1)}\right) \\
& \left.\times\left(\begin{array}{c}
j+\alpha \\
i+N
\end{array}\right)\right]_{0 \leq i, j \leq N-1} \\
= & {\left[\left(\begin{array}{c}
j+\alpha \\
i+N
\end{array}\right) \frac{(i)_{j \wedge 2}}{(j+\alpha-N)_{j \wedge 2}}\right]_{0 \leq i, j \leq N-1} . }
\end{aligned}
$$

This method can be recursively extended: apply the $k$ th transformation $(1 \leq k \leq N-1)$ defined, for $j \in\{k, \ldots, N-1\}$, by

$$
\begin{aligned}
\mathbf{C}_{j}^{(k)}= & \mathbf{C}_{j}^{(k-1)}-\frac{j+\alpha}{j+\alpha-N} \mathbf{C}_{j-1}^{(k-1)} \\
= & \mathbf{C}_{j}^{(k-2)}-2 \frac{j+\alpha}{j+\alpha-N} \mathbf{C}_{j-1}^{(k-2)} \\
& +\frac{(j+\alpha)(j+\alpha-1)}{(j+\alpha-N)(j+\alpha-N-1)} \mathbf{C}_{j-2}^{(k-2)} \\
& \vdots \\
= & \mathbf{C}_{j}^{(0)}-\left(\begin{array}{c}
k \\
1
\end{array}\right) \frac{j+\alpha}{j+\alpha-N} \mathbf{C}_{j-1}^{(0)}
\end{aligned}
$$

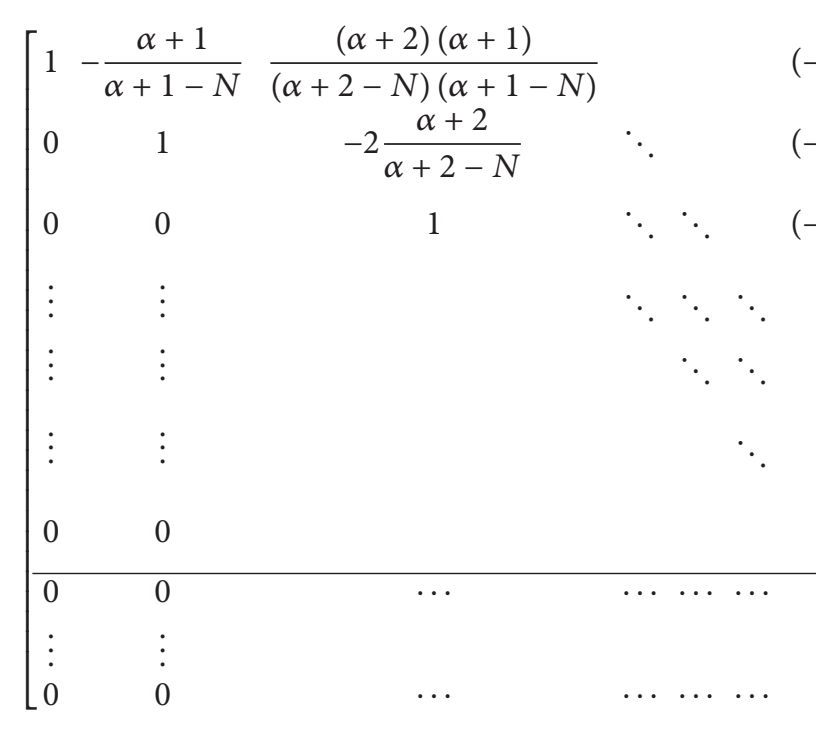

$$
\begin{aligned}
& +\left(\begin{array}{l}
k \\
2
\end{array}\right) \frac{(j+\alpha)(j+\alpha-1)}{(j+\alpha-N)(j+\alpha-N-1)} \mathbf{C}_{j-2}^{(0)} \\
& +\cdots+(-1)^{k}\left(\begin{array}{l}
k \\
k
\end{array}\right) \\
& \times \frac{(j+\alpha)(j+\alpha-1) \cdots(j+\alpha-k+1)}{(j+\alpha-N)(j+\alpha-N-1) \cdots(j+\alpha-k-N+1)} \\
& \times \mathbf{C}_{j-k}^{(0)} \\
= & \sum_{\ell=0}^{k}(-1)^{\ell}\left(\begin{array}{l}
k \\
\ell
\end{array}\right) \frac{(j+\alpha)_{\ell}}{(j+\alpha-N)_{\ell}} \mathbf{C}_{j-\ell}^{(0)} \\
= & \sum_{\ell=0}^{k}(-1)^{\ell}\left(\begin{array}{l}
k \\
\ell
\end{array}\right) \frac{(j+\alpha)_{N}}{(j+\alpha-\ell)_{N}} \mathbf{C}_{j-\ell}^{(0)} \\
= & \sum_{\ell=j-k}^{j}(-1)^{j-\ell}\left(\begin{array}{c}
k \\
j-\ell
\end{array}\right) \frac{(j+\alpha)_{j-\ell}}{(j+\alpha-N)_{j-\ell}} \mathbf{C}_{\ell}^{(0)} \\
= & \sum_{\ell=j-k}^{j}(-1)^{j-\ell}\left(\begin{array}{c}
k \\
j-\ell
\end{array}\right) \frac{(j+\alpha)_{N}}{(\ell+\alpha)_{N}} \mathbf{C}_{\ell}^{(0)} .
\end{aligned}
$$

In particular,

$$
\mathbf{C}_{k}^{(k)}=\sum_{\ell=0}^{k}(-1)^{k-\ell}\left(\begin{array}{l}
k \\
\ell
\end{array}\right) \frac{(k+\alpha)_{N}}{(\ell+\alpha)_{N}} \mathbf{C}_{\ell}^{(0)} .
$$

The $\mathbf{C}_{0}^{(0)}, \mathbf{C}_{1}^{(1)}, \mathbf{C}_{2}^{(2)}, \ldots, \mathbf{C}_{k}^{(k)}, \mathbf{C}_{k+1}^{(k)}, \ldots, \mathbf{C}_{N-1}^{(k)}$ are the columns

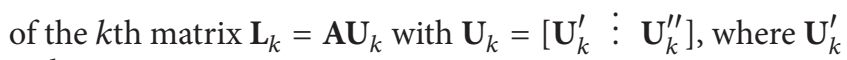
is the matrix

$$
\begin{gathered}
(-1)^{k-1}\left(\begin{array}{l}
k-1 \\
k-1
\end{array}\right) \frac{(\alpha+k-1) \cdots(\alpha+1)}{(\alpha+k-N-1) \cdots(\alpha+1-N)} \\
(-1)^{k-2}\left(\begin{array}{l}
k-1 \\
k-2
\end{array}\right) \frac{(\alpha+k-1) \cdots(\alpha+2)}{(\alpha+k-N-1) \cdots(\alpha+2-N)} \\
(-1)^{k-3}\left(\begin{array}{l}
k-1 \\
k-3
\end{array}\right) \frac{(\alpha+k-1) \cdots(\alpha+3)}{(\alpha+k-N-1) \cdots(\alpha+3-N)} \\
\vdots \\
\left(\begin{array}{c}
k-1 \\
2
\end{array}\right) \frac{(\alpha+k-1)(\alpha+k-2)}{(\alpha+k-N-1)(\alpha+k-N-2)} \\
-\left(\begin{array}{c}
k-1) \frac{\alpha+k-1}{1} \\
\alpha+k-N-1 \\
k
\end{array}\right)
\end{gathered}
$$

and $\mathbf{U}_{k}^{\prime \prime}$ is the matrix 


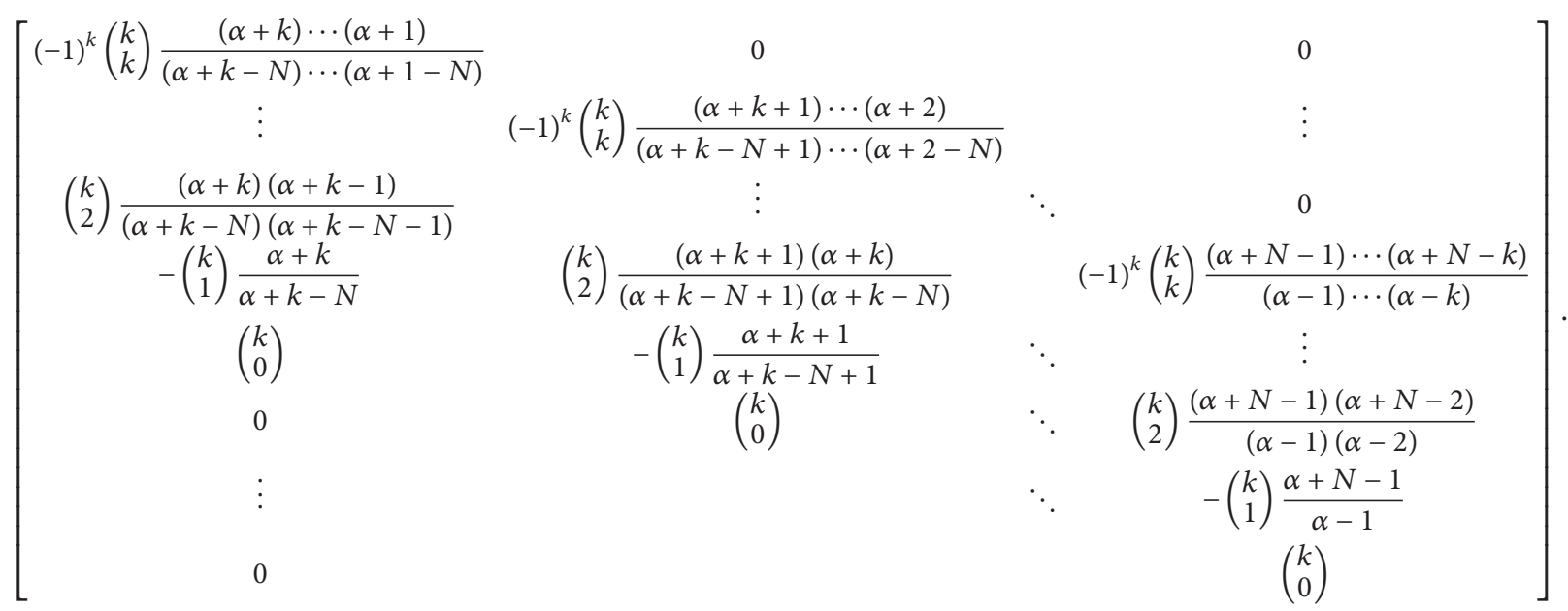

The matrices $\mathbf{U}_{k}^{\prime}$ and $\mathbf{U}_{k}^{\prime \prime}$ can be simply written as

$$
\begin{gathered}
\mathbf{U}_{k}^{\prime}=\left[(-1)^{j-i} \mathbb{1}_{\{i \leq j\}}\left(\begin{array}{c}
j \\
i
\end{array}\right) \frac{(j+\alpha)_{N}}{(i+\alpha)_{N}}\right]_{\substack{0 \leq i \leq N-1 \\
0 \leq j \leq k-1}}, \\
\mathbf{U}_{k}^{\prime \prime}=\left[(-1)^{j-i} \mathbb{1}_{\{i \vee k \leq j \leq i+k\}}\left(\begin{array}{c}
k \\
j-i
\end{array}\right) \frac{(j+\alpha)_{N}}{(i+\alpha)_{N}}\right]_{\substack{0 \leq i \leq N-1 \\
k \leq j \leq N-1}} .
\end{gathered}
$$

Clever algebra yields that

$$
\mathbf{L}_{k}=\left[\left(\begin{array}{c}
j+\alpha \\
i+N
\end{array}\right) \frac{(i)_{j \wedge k}}{(j+\alpha-N)_{j \wedge k}}\right]_{0 \leq i, j \leq N-1}
$$

We will not prove (A.53); we will only check it below in the case $k=N-1$.

We progressively arrive at the last transformation which corresponds to $k=N-1$ :

$$
\begin{aligned}
\mathbf{C}_{N-1}^{(N-1)}= & \mathbf{C}_{N-1}^{(0)}-\left(\begin{array}{c}
N-1 \\
1
\end{array}\right) \frac{N+\alpha-1}{\alpha-1} \mathbf{C}_{N-2}^{(0)} \\
& +\left(\begin{array}{c}
N-1 \\
2
\end{array}\right) \frac{(N+\alpha-1)(N+\alpha-2)}{(\alpha-1)(\alpha-2)} \mathbf{C}_{N-3}^{(0)} \\
& +(-1)^{N-1}\left(\begin{array}{c}
N-1 \\
N-1
\end{array}\right) \\
& \times \frac{(N+\alpha-1)(N+\alpha-2) \cdots(\alpha+1)}{(\alpha-1)(\alpha-2) \cdots(\alpha-N+1)} \mathbf{C}_{0}^{(0)} \\
= & \sum_{\ell=0}^{N-1}(-1)^{\ell}\left(\begin{array}{c}
N-1 \\
\ell
\end{array}\right) \frac{(N+\alpha-1)_{\ell}}{(\alpha-1)_{\ell}} \mathbf{C}_{N-1-\ell}^{(0)}
\end{aligned}
$$

$$
\begin{gathered}
=\sum_{\ell=0}^{N-1}(-1)^{N-1-\ell}\left(\begin{array}{c}
N-1 \\
\ell
\end{array}\right) \\
\quad \times \frac{(N+\alpha-1)_{N-\ell-1}}{(\alpha-1)_{N-\ell-1}} \mathbf{C}_{\ell}^{(0)} .
\end{gathered}
$$

The $\mathbf{C}_{0}^{(0)}, \mathbf{C}_{1}^{(1)}, \mathbf{C}_{2}^{(2)}, \ldots, \mathbf{C}_{N-1}^{(N-1)}$ are the columns of the last matrix given by $\mathbf{L}_{N-1}=\mathbf{A} \mathbf{U}_{N-1}$, where

$$
\mathbf{U}_{N-1}=\left[(-1)^{j-i}\left(\begin{array}{l}
j \\
i
\end{array}\right) \frac{(j+\alpha)_{N}}{(i+\alpha)_{N}}\right]_{0 \leq i, j \leq N-1} .
$$

Formula (A.53) gives the following expression for $\mathbf{L}_{N-1}$ that will be checked below:

$$
\mathbf{L}_{N-1}=\left[\left(\begin{array}{c}
j+\alpha \\
i+N
\end{array}\right) \frac{(i)_{j}}{(j+\alpha-N)_{j}}\right]_{0 \leq i, j \leq N-1} .
$$

Hence, by putting $\mathbf{L}=\mathbf{L}_{N-1}$ and $\mathbf{U}=\mathbf{U}_{N-1}$, we see that $\mathbf{L}$ is a lower triangular matrix and $\mathbf{U}$ is an upper triangular matrix and we have obtained that $\mathbf{L}=\mathbf{A U}$.

Finally, we directly check the decomposition $\mathbf{L}=\mathbf{A U}$. The generic term of $\mathbf{A U}$ is

$$
\sum_{k=0}^{j}(-1)^{j+k}\left(\begin{array}{l}
k+\alpha \\
i+N
\end{array}\right)\left(\begin{array}{l}
j \\
k
\end{array}\right) \frac{(j+\alpha)_{N}}{(k+\alpha)_{N}} .
$$

Observing that

$$
\left(\begin{array}{c}
k+\alpha \\
i+N
\end{array}\right) \frac{(j+\alpha)_{N}}{(k+\alpha)_{N}}=\frac{\left(\begin{array}{c}
k+\alpha-N \\
i
\end{array}\right)\left(\begin{array}{c}
j+\alpha \\
N
\end{array}\right)}{\left(\begin{array}{c}
i+N \\
N
\end{array}\right)},
$$

this term can be rewritten as

$$
\frac{(-1)^{j}\left(\begin{array}{c}
j+\alpha \\
N
\end{array}\right)}{\left(\begin{array}{c}
i+N \\
N
\end{array}\right)} \times \sum_{k=0}^{j}(-1)^{k}\left(\begin{array}{l}
j \\
k
\end{array}\right)\left(\begin{array}{c}
k+\alpha-N \\
i
\end{array}\right) .
$$


The sum $\sum_{k=0}^{j}(-1)^{k}\left(\begin{array}{c}j \\ k\end{array}\right)\left(\begin{array}{c}k+\alpha-N \\ i\end{array}\right)$ can be explicitly evaluated thanks to Lemma A.4. Its value is $(-1)^{j}\left(\begin{array}{c}\alpha-N \\ i-j\end{array}\right)$. Therefore, we can easily get the generic term of $\mathbf{L}$, and the proof of Lemma A.5 is finished.

Lemma A.6. The inverse of the matrix $\mathbf{L}$ is given by

$$
\mathbf{L}^{-1}=\left[(-1)^{i+j} \mathbb{1}_{\{i \geq j\}} \frac{(j+N) !(i+\alpha-N)_{i+1}}{j !(i-j) !(i+\alpha)_{j+N+1}}\right]_{0 \leq i, j \leq N-1}
$$

Proof. We simplify the entries of the product

$$
\begin{aligned}
& {\left[\mathbb{1}_{\{i \geq j\}}\left(\begin{array}{c}
j+\alpha \\
i+N
\end{array}\right) \frac{(i)_{j}}{(j+\alpha-N)_{j}}\right]_{0 \leq i, j \leq N-1}} \\
& \quad \times\left[(-1)^{i+j} \mathbb{1}_{\{i \geq j\}} \frac{(j+N) !(i+\alpha-N)_{i+1}}{j !(i-j) !(i+\alpha)_{j+N+1}}\right]_{0 \leq i, j \leq N-1} .
\end{aligned}
$$

The generic term of this matrix is

$$
\begin{aligned}
\sum_{k=0}^{N-1} \mathbb{1}_{\{i \geq k\}}\left(\begin{array}{c}
k+\alpha \\
i+N
\end{array}\right) \frac{(i)_{k}}{(k+\alpha-N)_{k}} \\
\quad \times(-1)^{j+k} \mathbb{1}_{\{k \geq j\}} \frac{(j+N) !(k+\alpha-N)_{k+1}}{j !(k-j) !(k+\alpha)_{j+N+1}} \\
=\mathbb{1}_{\{i \geq j\}} \frac{i !(j+N) !(\alpha-N) !}{j !(i+N) !(\alpha-N-1) !} \\
\quad \times \sum_{k=j}^{i}(-1)^{j+k} \frac{(k+\alpha-j-N-1) !}{(i-k) !(k-j) !(k+\alpha-i-N) !} .
\end{aligned}
$$

The last sum can be computed as follows: clearly, it vanishes when $i<j$ and it equals 1 when $i=j$. If $i>j$, by using Lemma A.4,

$$
\begin{aligned}
& \sum_{k=j}^{i}(-1)^{j+k} \frac{(k+\alpha-j-N-1) !}{(i-k) !(k-j) !(k+\alpha-i-N) !} \\
& \quad=\frac{1}{(i-j) !} \sum_{k=j}^{i}(-1)^{j+k}\left(\begin{array}{c}
i-j \\
k-j
\end{array}\right) \frac{(k+\alpha-j-N-1) !}{(k+\alpha-i-N) !} \\
& =\frac{1}{(i-j) !} \sum_{k=0}^{i-j}(-1)^{k}\left(\begin{array}{c}
i-j \\
k
\end{array}\right)(k+\alpha-N-1)_{i-j-1}=0 .
\end{aligned}
$$

As a consequence, the entries of the product (A.61) are $\delta_{i j}$ which proves that the second factor of (A.61) coincides with $\mathbf{L}^{-1}$.

Lemma A.7. The matrix $\mathbf{L}^{-1} \mathbf{B}$ is given by

$$
\mathbf{L}^{-1} \mathbf{B}=\left[\frac{(-1)^{i}\left(\begin{array}{c}
\beta \\
N
\end{array}\right)\left(\begin{array}{c}
i+\alpha-\beta-1 \\
\alpha-\beta-1
\end{array}\right)}{\left(\begin{array}{c}
i+\alpha \\
N
\end{array}\right)}\right]_{0 \leq i \leq N-1} .
$$

Proof. The generic term of $\mathbf{L}^{-1} \mathbf{B}$ is

$$
\begin{aligned}
\sum_{j=0}^{N-1}(-1)^{i+j} \mathbb{1}_{\{i \geq j\}} \frac{(j+N) !(i+\alpha-N)_{i+1}}{j !(i-j) !(i+\alpha)_{j+N+1}}\left(\begin{array}{c}
\beta \\
j+N
\end{array}\right) & \frac{\beta !(i+\alpha-N) !}{(i+\alpha) !(\alpha-N-1) !} \\
& \times \sum_{j=0}^{i}(-1)^{i+j} \frac{(i-j+\alpha-N-1) !}{j !(i-j) !(\beta-j-N) !} \\
= & \frac{\beta !(i+\alpha-N) !(i+\alpha-\beta-1) !}{i !(i+\alpha) !(\alpha-N-1) !} \\
& \times \sum_{j=0}^{i}(-1)^{i+j}\left(\begin{array}{c}
i \\
j
\end{array}\right)\left(\begin{array}{c}
i-j+\alpha-N-1 \\
i+\alpha-\beta-1
\end{array}\right) .
\end{aligned}
$$

By performing the change of index $j \mapsto i-j$ and by using Lemma A.4, the sum in (A.65) is equal to

$$
\begin{aligned}
\sum_{j=0}^{i}(-1)^{j}\left(\begin{array}{l}
i \\
j
\end{array}\right)\left(\begin{array}{c}
j+\alpha-N-1 \\
i+\alpha-\beta-1
\end{array}\right) & =(-1)^{i}\left(\begin{array}{c}
\alpha-N-1 \\
\beta-N
\end{array}\right) \\
& =(-1)^{i} \frac{(\alpha-N-1) !}{(\alpha-\beta-1) !(\beta-N) !} .
\end{aligned}
$$

By putting this into (A.65), we see that the generic term of $\mathbf{L}^{-1} \mathbf{B}$ writes

$$
(-1)^{i} \frac{\beta !(i+\alpha-N) !(i+\alpha-\beta-1) !}{i !(i+\alpha) !(\alpha-\beta-1) !(\beta-N) !}=\frac{(-1)^{i}\left(\begin{array}{c}
\beta \\
N
\end{array}\right)\left(\begin{array}{c}
i+\alpha-\beta-1 \\
\alpha-\beta-1
\end{array}\right)}{\left(\begin{array}{c}
i+\alpha \\
N
\end{array}\right)}
$$

which ends up the proof of Lemma A.7.

Theorem A.8. The matrix $\mathbf{A}^{-1} \mathbf{B}$ is given by

$$
\mathbf{A}^{-1} \mathbf{B}=\left[(-1)^{i} \frac{N}{i+\alpha-\beta} \frac{\left(\begin{array}{c}
\beta \\
N
\end{array}\right)\left(\begin{array}{c}
\alpha-\beta+N-1 \\
N
\end{array}\right)\left(\begin{array}{c}
N-1 \\
i
\end{array}\right)}{\left(\begin{array}{c}
i+\alpha \\
N
\end{array}\right)}\right]_{0 \leq i \leq N-1}
$$

Proof. Referring to Lemmas A.5 and A.7, we have that

$$
\begin{aligned}
\mathbf{A}^{-1} \mathbf{B}= & \mathbf{U}\left(\mathbf{L}^{-1} \mathbf{B}\right) \\
= & {\left[\frac{(-1)^{i+j} \mathbb{1}_{\{i \leq j\}}\left(\begin{array}{c}
j \\
i
\end{array}\right)\left(\begin{array}{c}
j+\alpha \\
N
\end{array}\right)}{\left(\begin{array}{c}
i+\alpha \\
N
\end{array}\right)}\right]_{0 \leq i, j \leq N-1} } \\
& \times\left[\frac{(-1)^{i}\left(\begin{array}{c}
\beta \\
N
\end{array}\right)\left(\begin{array}{c}
i+\alpha-\beta-1 \\
\alpha-\beta-1
\end{array}\right)}{\left(\begin{array}{c}
i+\alpha \\
N
\end{array}\right)}\right]_{0 \leq i \leq N-1} .
\end{aligned}
$$

The generic term of $\mathbf{A}^{-1} \mathbf{B}$ is

$$
\frac{(-1)^{i}\left(\begin{array}{c}
\beta \\
N
\end{array}\right)}{\left(\begin{array}{c}
i+\alpha \\
N
\end{array}\right)} \sum_{j=i}^{N-1}\left(\begin{array}{c}
j \\
i
\end{array}\right)\left(\begin{array}{c}
j+\alpha-\beta-1 \\
\alpha-\beta-1
\end{array}\right) .
$$


TABLE 2: List of notations

\begin{tabular}{|c|c|}
\hline$a, b, c, K, N, \kappa_{N}, p_{k}$ & Given parameters \\
\hline$\theta_{j}, \varphi_{j}$ & Roots of \pm 1 \\
\hline$\xi_{k}, S_{n}, X_{t}, X_{t}^{\varepsilon}$ & Pseudorandom variables, pseudoprocesses \\
\hline $\mathscr{G}_{s}, \mathscr{G}_{X}$ & Infinitesimal generators \\
\hline$\sigma_{a}^{-}, \sigma_{b}^{+}, \sigma_{a b}, S_{a}^{-}, S_{b}^{+}, S_{a b}$ & Overshooting times and locations related to pseudorandom walk \\
\hline $\begin{array}{l}\tau_{a}^{-}, \tau_{b}^{+}, \tau_{a b}, X_{a}^{-}, X_{b}^{+}, X_{a b} \\
\tau_{a}^{\varepsilon-}, \tau_{b}^{\varepsilon+}, \tau_{a b}^{\varepsilon}, X_{a}^{\varepsilon-}, X_{b}^{\varepsilon+}, X_{a b}^{\varepsilon}\end{array}$ & Overshooting times and locations related to pseudo-Brownian motion \\
\hline$\Delta, \Delta^{+}, \Delta^{-}$ & Discrete Laplacian, finite difference operators \\
\hline $\begin{array}{l}A\left(a_{1}, \ldots, a_{N}\right), A_{k}\left(\begin{array}{c}a_{1}, \ldots, a_{N} \\
\alpha_{1}, \ldots, \alpha_{N}\end{array}\right) \\
U\left(u_{1}, \ldots, u_{2 N}\right), U_{\ell}\left(u_{1}, \ldots, u_{2 N}\right) \\
U_{k \ell}\left(u_{1}, \ldots, u_{k-1}, u_{k+1}, \ldots, u_{2 N}\right) \\
U_{p q r}\left(u_{1}, \ldots, u_{p+r}\right), U_{p q r, \ell}\left(\begin{array}{c}u_{1}, \ldots, u_{p+r} \\
\alpha_{1}, \ldots, \alpha_{p+r}\end{array}\right) \\
U_{p q r, k \ell}\left(u_{1}, \ldots, u_{k-1}, u_{k+1}, \ldots, u_{p+r}\right) \\
V\left(v_{1}, \ldots, v_{N}\right), V_{\ell}\left(v_{1}, \ldots, v_{N}\right) \\
V_{k \ell}\left(v_{1}, \ldots, v_{k-1}, v_{k+1}, \ldots, v_{N}\right) \\
\widetilde{U}(z), \widetilde{U}_{k \ell}(z), \widetilde{V}(z), \widetilde{V}_{k}(z), D(z), D_{k}(z, \zeta) \\
\mathbf{D}(\lambda), \mathbf{D}_{k}^{+}(\lambda, \mu), \mathbf{D}_{k}^{-}(\lambda, \mu)\end{array}$ & Vandermonde-like determinants \\
\hline $\mathcal{S}_{p q r}, \mathcal{S}_{p q r, k \ell}$ & Other determinants \\
\hline$G_{k}(z), G(z, \zeta), H_{a, \ell}^{-}(z), H_{b, \ell}^{+}(z), H_{a b, \ell}(z)$ & Generating functions \\
\hline $\begin{array}{l}L_{k}(z, \zeta), \widetilde{L}_{k}(z, \zeta), P(z, \zeta), P_{k}(z, \zeta) \\
P_{b, j}^{+}(x), P_{a b, j}(x), K_{m}(x), \widetilde{K}_{m}(x)\end{array}$ & Polynomials \\
\hline$I_{a b, j}^{+}, I_{a b, j}^{-}, \mathbf{I}_{a b, j}^{+}, \mathbf{I}_{a b, j}^{-}$ & Integrals and sums \\
\hline $\mathscr{D}^{+}, \mathscr{D}^{-}, \mathscr{E}$ & Sets \\
\hline$M_{1}, M_{\infty}$ & Bounds \\
\hline$a_{j}(z), b_{j}(z), u_{j}(z), v_{j}(z), w(z)$ & \\
\hline $\begin{array}{l}\alpha_{j}(z), \widetilde{\alpha}_{j}(z), \epsilon_{j}, \varepsilon_{j}(z), \mathrm{u}_{j}(z), \mathrm{v}_{j}(z) \\
A_{j}(z), B_{j}(z), M_{k}(z), R_{j}(z), a_{\varepsilon}, b_{\varepsilon}\end{array}$ & Miscellaneous \\
\hline $\begin{array}{l}s_{\ell}(z), p_{k}(z), s_{k, \ell}^{+}(z), p_{k}^{+}(z), s_{k, \ell}^{-}(z), p_{k}^{-}(z) \\
s_{k, m}\left(u_{1}, \ldots, u_{k-1}, u_{k+1}, \ldots, u_{p+r}\right)\end{array}$ & Sums and products \\
\hline $\mathbf{A}, \mathbf{B}, \mathbf{C}_{i}^{(k)}, \mathbf{L}, \mathbf{U}, \mathbf{U}^{\prime}, \mathbf{U}^{\prime \prime}$ & Matrices \\
\hline$\vec{V}_{m}$ & Vectors \\
\hline
\end{tabular}

The foregoing sum can be easily evaluated as follows:

$$
\begin{aligned}
& \sum_{j=i}^{N-1}\left(\begin{array}{l}
j \\
i
\end{array}\right)\left(\begin{array}{c}
j+\alpha-\beta-1 \\
\alpha-\beta-1
\end{array}\right) \\
&=\left(\begin{array}{c}
i+\alpha-\beta-1 \\
\alpha-\beta-1
\end{array}\right) \sum_{j=i}^{N-1}\left(\begin{array}{c}
j+\alpha-\beta-1 \\
i+\alpha-\beta-1
\end{array}\right) \\
&=\left(\begin{array}{c}
i+\alpha-\beta-1 \\
\alpha-\beta-1
\end{array}\right) \\
&=\left(\begin{array}{c}
i+\alpha-\beta-1 \\
\alpha-\beta-1
\end{array}\right)\left(\begin{array}{c}
\alpha-\beta+N-1 \\
i+\alpha-\beta
\end{array}\right) \\
&= \frac{N}{i+\alpha-\beta}\left(\begin{array}{c}
\alpha-\beta+N-1 \\
N
\end{array}\right)\left(\begin{array}{c}
N-1 \\
i
\end{array}\right) .
\end{aligned}
$$

Putting this into (A.70) yields the matrix $\mathbf{A}^{-1} \mathbf{B}$ displayed in Theorem A.8.

\section{Conflict of Interests}

The author declares that there is no conflict of interests regarding the publication of this paper.

\section{References}

[1] L. Beghin, K. J. Hochberg, and E. Orsingher, "Conditional maximal distributions of processes related to higher-order heattype equations," Stochastic Processes and their Applications, vol. 85 , no. 2, pp. 209-223, 2000.

[2] L. Beghin and E. Orsingher, "The distribution of the local time for "pseudoprocesses" and its connection with fractional diffusion equations," Stochastic Processes and their Applications, vol. 115, no. 6, pp. 1017-1040, 2005. 
[3] L. Beghin, E. Orsingher, and T. Ragozina, "Joint distributions of the maximum and the process for higher-order diffusions," Stochastic Processes and their Applications, vol. 94, no. 1, pp. 7193, 2001.

[4] V. Cammarota and A. Lachal, "Joint distribution of the process and its sojourn time on the positive half-line for pseudoprocesses governed by high-order heat equation," Electronic Journal of Probability, vol. 15, pp. 895-931, 2010.

[5] V. Cammarota and A. Lachal, "Joint distribution of the process and its sojourn time in a half-line $[a,+\infty)$ for pseudo-processes driven by a high-order heat-type equation," Stochastic Processes and their Applications, vol. 122, no. 1, pp. 217-249, 2011.

[6] K. J. Hochberg, "A signed measure on path space related to Wiener measure," Annals of Probability, vol. 6, no. 3, pp. 433458, 1978.

[7] V. Yu. Krylov, "Some properties of the distribution corresponding to the equation $\partial u / \partial t=(-1)^{q+1}\left(\partial^{2 q} u / \partial^{2 q} x\right)$," Soviet Mathematics, pp. 760-763, 1960.

[8] K. J. Hochberg and E. Orsingher, "The arc-sine law and its analogs for processes governed by signed and complex measures," Stochastic Processes and their Applications, vol. 52, no. 2, pp. 273-292, 1994.

[9] A. Lachal, "Distributions of Sojourn time, maximum and minimum for pseudo-processes governed by higher-order heattype equations," Electronic Journal of Probability, vol. 8, pp. 1-53, 2003.

[10] A. Lachal, "First hitting time and place, monopoles and multipoles for pseudo-processes driven by the equation $\partial / \partial t=$ $\pm \partial^{N} / \partial x^{N}$, , Electronic Journal of Probability, vol. 12, pp. 300-353, 2007.

[11] A. Lachal, "Joint law of the process and its maximum, first hitting time and place of a half-line for the pseudo-process driven by the equation $\partial / \partial t= \pm \partial^{N} / \partial x^{N}$," Comptes Rendus Mathematique, vol. 343, no. 8, pp. 525-530, 2006.

[12] A. Lachal, "First hitting time and place for pseudo-processes driven by the equation $\partial / \partial t= \pm \partial^{n} / \partial x^{n}$ subject to a linear drift," Stochastic Processes and their Applications, vol. 118, no. 1, pp. 127, 2008.

[13] A. Lachal, "A survey on the pseudo-process driven by the high-order heat-type equation $\partial / \partial t= \pm \partial^{N} / \partial x^{N}$ concerning the hitting and sojourn times," Methodology and Computing in Applied Probability, pp. 1-18, 2011.

[14] A. Lachal, "First exit time from a bounded interval for pseudoprocesses driven by the equation $\partial / \partial t=(-1)^{N-1} \partial^{2 N} / \partial x^{N}$," Stochastic Processes and their Applications, vol. 124, no. 2, pp. 1084-1111, 2014.

[15] T. Nakajima and S. Sato, "On the joint distribution of the first hitting time and the first hitting place to the space-time wedge domain of a biharmonic pseudo process," Tokyo Journal of Mathematics, vol. 22, no. 2, pp. 399-413, 1999.

[16] Y. Nikitin and E. Orsingher, "On sojourn distributions of processes related to some higher-order heat-type equations," Journal of Theoretical Probability, vol. 13, no. 4, pp. 997-1012, 2000.

[17] K. Nisiiioka, "Monopoles and dipoles in biharmonic pseudo process," Proceedings of the Japan Academy Series A, vol. 72, no. 3, pp. 47-50, 1996.

[18] K. Nishioka, "The first hitting time and place of a half-line by a biharmonic pseudo process," Japanese Journal of Mathematics, vol. 23, pp. 235-280, 1997.
[19] K. Nishioka, "Boundary conditions for one-dimensional biharmonic pseudo process," Electronic Journal of Probability, vol. 6, pp. 1-27, 2001.

[20] E. Orsingher, "Processes governed by signed measures connected with third-order "heat-type" equations," Lithuanian Mathematical Journal, vol. 31, no. 2, pp. 220-231, 1991.

[21] S. Sato, "An approach to the biharmonic pseudo process by a random walk," Journal of Mathematics of Kyoto University, vol. 42, no. 3, pp. 403-422, 2002.

[22] R. J. Vanderbei, "Probabilistic solution of the Dirichlet problem for biharmonic functions in discrete space," Annals of Probability, vol. 12, no. 2, pp. 311-324, 1984. 


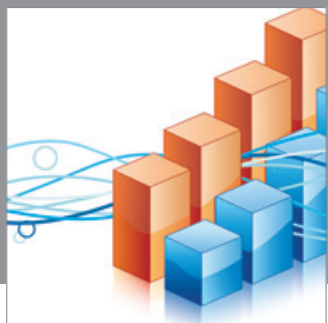

Advances in

Operations Research

mansans

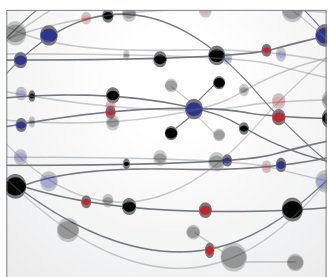

The Scientific World Journal
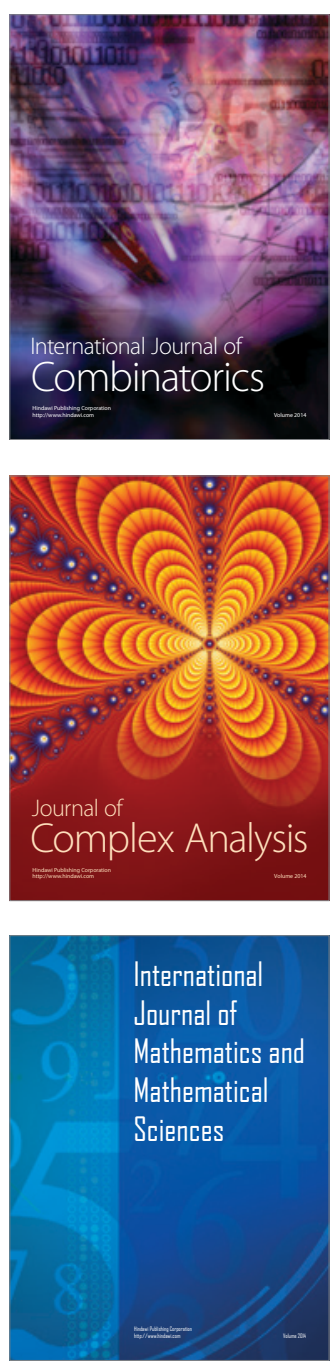
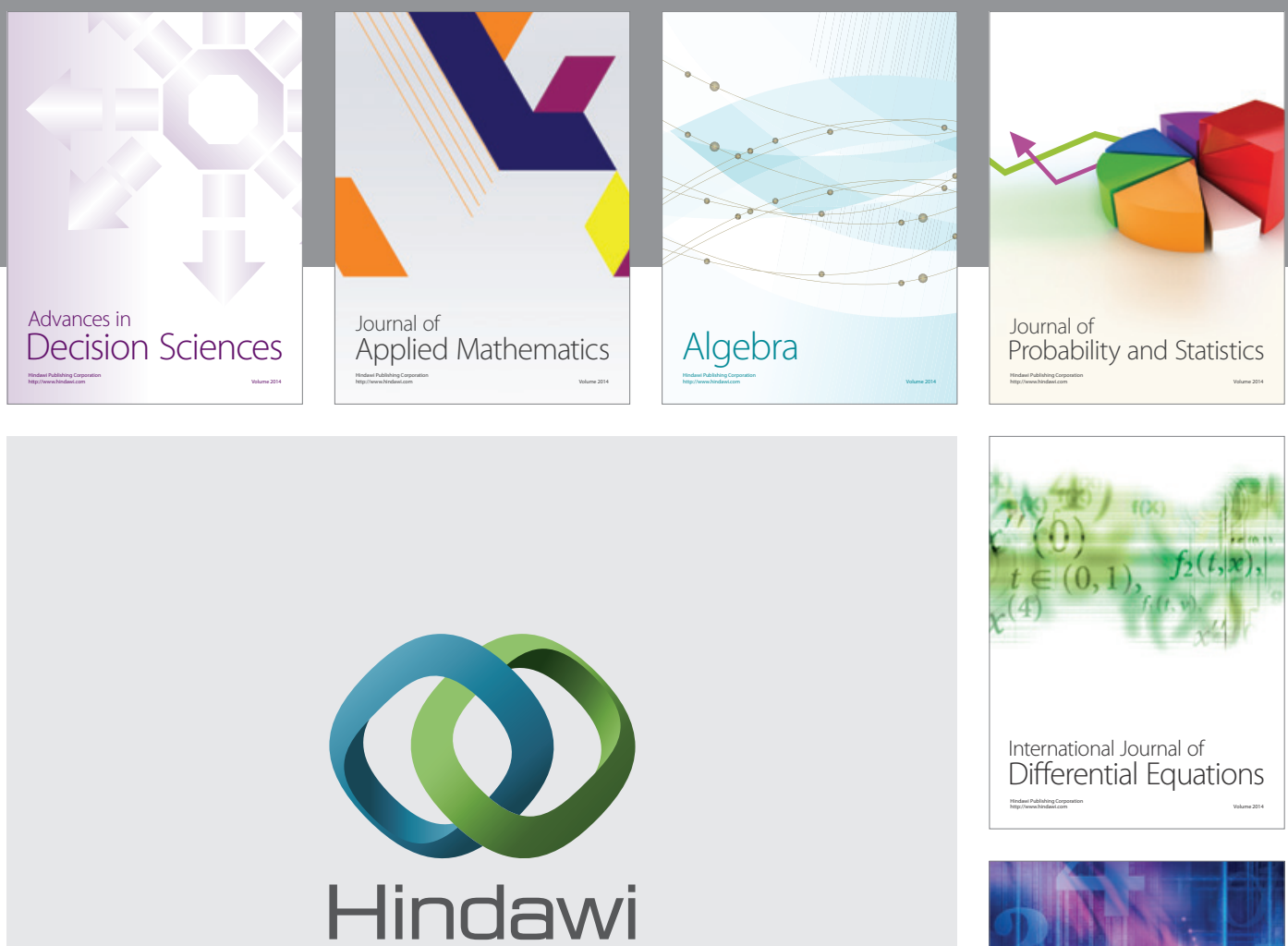

Submit your manuscripts at http://www.hindawi.com
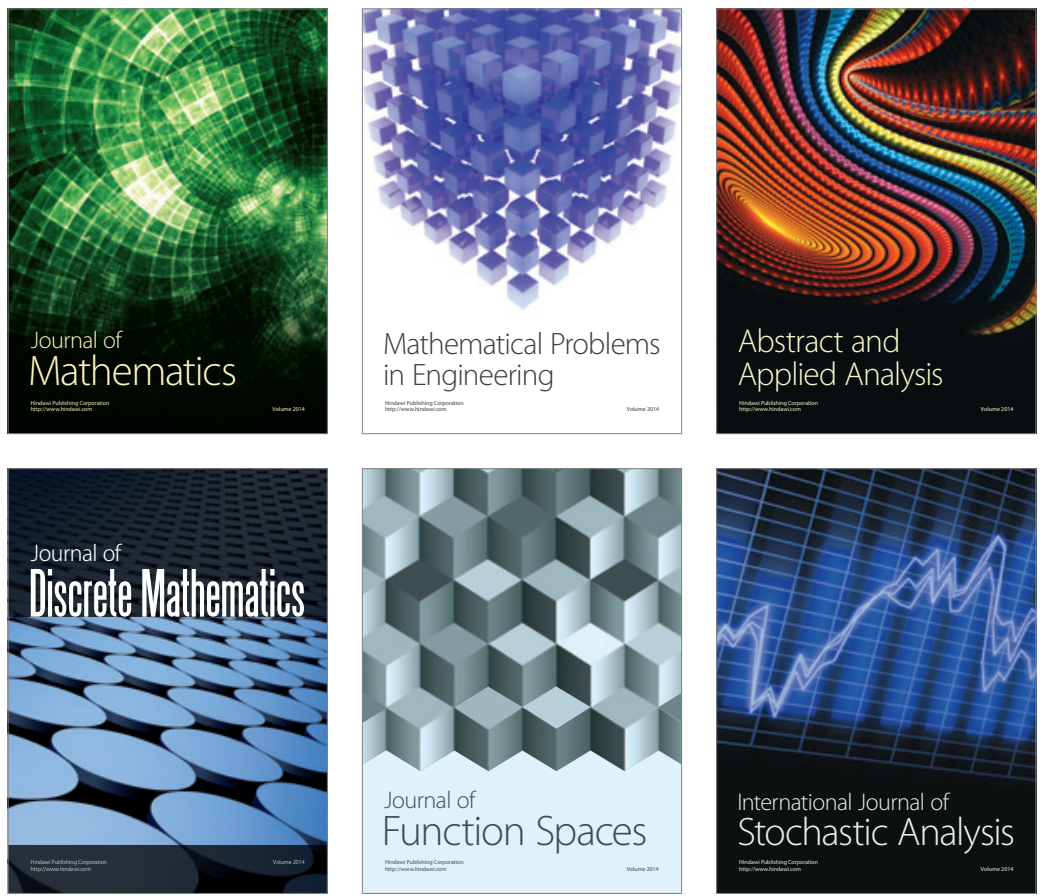

Journal of

Function Spaces

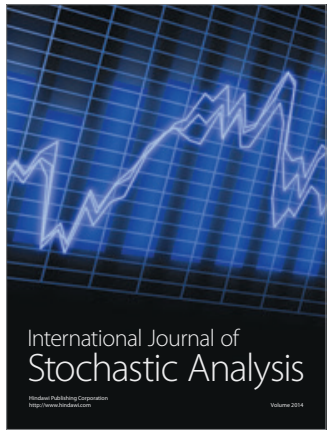

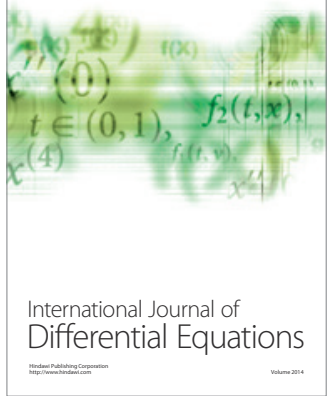
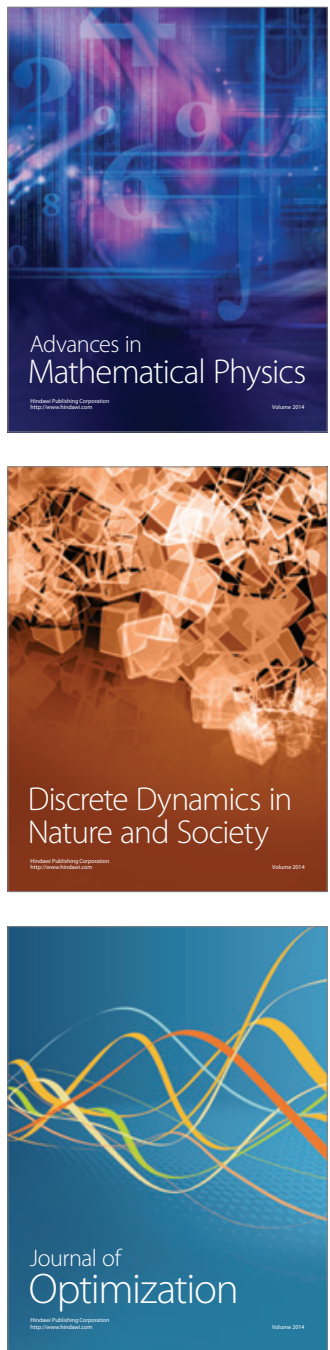